\title{
On entropy production of repeated quantum measurements I. General theory
}

\author{
T. Benoist ${ }^{1}$, V. Jakšić ${ }^{2}$, Y. Pautrat ${ }^{3}$, C-A. Pillet ${ }^{4}$ \\ ${ }^{1}$ CNRS, Laboratoire de Physique Théorique, IRSAMC, \\ Université de Toulouse, UPS, F-31062 Toulouse, France \\ ${ }^{2}$ Department of Mathematics and Statistics, McGill University, \\ 805 Sherbrooke Street West, Montreal, QC, H3A 2K6, Canada \\ ${ }^{3}$ Laboratoire de Mathématiques d'Orsay, Univ. Paris-Sud, CNRS, \\ Université Paris-Saclay, 91405 Orsay, France \\ ${ }^{4}$ Aix Marseille Univ, Univ Toulon, CNRS, CPT, Marseille, France
}

\section{Dedicated to the memory of Rudolf Haag}

\begin{abstract}
We study entropy production (EP) in processes involving repeated quantum measurements of finite quantum systems. Adopting a dynamical system approach, we develop a thermodynamic formalism for the EP and study fine aspects of irreversibility related to the hypothesis testing of the arrow of time. Under a suitable chaoticity assumption, we establish a Large Deviation Principle and a Fluctuation Theorem for the EP.
\end{abstract}

\section{Contents}

1 Introduction $\quad 2$

1.1 Historical perspective ............................ 2

1.2 Setting .............................. 3

1.3 Two remarks ............................ 7

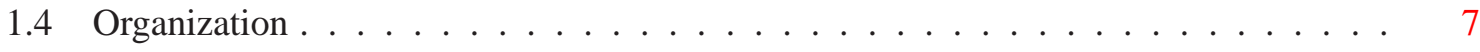

2 Results $\quad 8$

2.1 Level I: Entropies . . . . . . . . . . . . . . . . . . . . . . . . . . . . . . . . . . 8

2.2 Level I: Entropy production rate . . . . . . . . . . . . . . . . . . . . . 10 
2.3 Level I: Stein's error exponents . . . . . . . . . . . . . . . . . . . . . . . . . 14

2.4 Level II: Entropies . . . . . . . . . . . . . . . . . . . . . . . . . . . . 15

2.5 Level II: Rényi's relative entropy and thermodynamic formalism on $[0,1] \ldots \ldots$

2.6 Differentiability on $] 0,1[\ldots \ldots \ldots \ldots \ldots$

2.7 Full thermodynamic formalism . . . . . . . . . . . . . . . . . 22

2.8 Level II: Large Deviations _. . . . . . . . . . . . . . . . . . . . . 23

2.8 .1 Basic large deviations estimates . . . . . . . . . . . . . . 23

2.8 .2 A local and a global Fluctuation Theorem . . . . . . . . . . . . . . 24

2.9 Level II: Hypothesis testing . . . . . . . . . . . . . . . . . . . . . 25

3 Level I: Proofs. $\quad 28$

3.1 Proof of Theorem $2.1 \ldots \ldots \ldots \ldots \ldots \ldots$

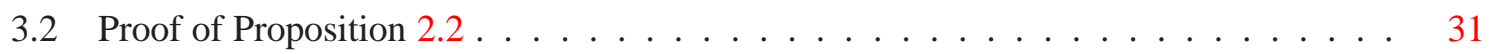

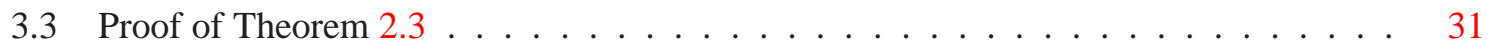

4 Level II: Proofs. $\quad 32$

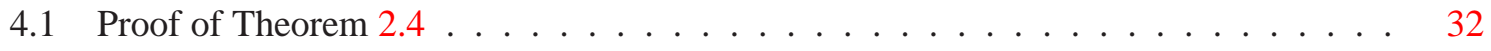

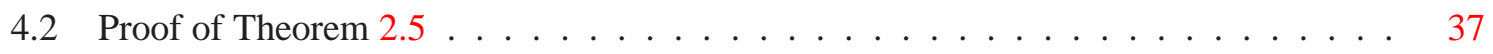

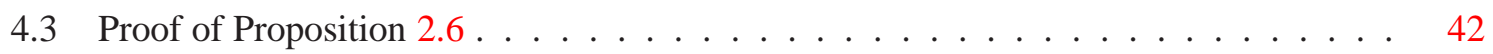

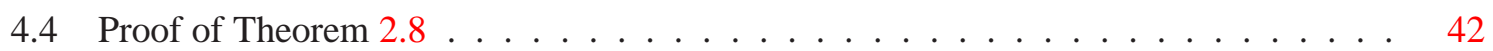

4.5 Proof of Proposition $2.9 \ldots \ldots \ldots \ldots \ldots \ldots \ldots$

4.6 Proof of Theorem $2.12 \ldots \ldots \ldots \ldots \ldots \ldots$

\section{Introduction}

\subsection{Historical perspective}

In 1927, invoking the "dephasing effect" of the interaction of a system with a measurement apparatus, Heisenberg $[\mathrm{He}]$ introduced the "reduction of the wave function" into quantum theory as the proper way to assign a wave function to a quantum system after a successful measurement. In the same year, Eddington [Ed] coined the term "arrow of time" in his discussion of the various aspects of irreversibility in physical systems. Building on Heisenberg's work, in his 1932 monograph [VN], von Neumann developed the first mathematical theory of quantum measurements. In this theory, the wave function reduction leads to an intrinsic irreversibility of the measurement process that has no classical analog and is sometimes called the quantum arrow of time. Although a consensus has been reached around the so-called orthodox ${ }^{1}$ approach to quantum measurements [Wi], after nearly a century of research, their fundamental status within quantum mechanics and their problematic relationship with "the observer" are far from understood and remain much debated; see [HMPZ, ST, Ze, BFFS, BFS].

\footnotetext{
${ }^{1}$ sometimes facetiously termed the "shut up and calculate" approach; see [MND].
} 
On entropy production of repeated quantum measurements I

Regarding the quantum arrow of time, Bohm [Bo, Section 22.12] points out that "[quantum] irreversibility greatly resembles that which appears in thermodynamic processes," while Landau and Lifshitz [LL, Section I.8] go further and discuss the possibility that the second law of thermodynamics and the thermodynamical arrow of time are macroscopic expressions of the quantum arrow of time. In 1964, Aharonov, Bergmann and Lebowitz [ABL] critically examined the nature of the quantum arrow of time. They showed that conditioning on both the initial and final quantum states, one could construct a time symmetric statistical ensemble of quantum measurements, lifting the problem of time irreversibility implied by the projection postulate to a question of appropriate choice of a statistical ensemble. The construction of this "two-state vector formalism" [AV] and weak measurements [BG, Ca, Da, WM] has led to the definition of weak values by Aharonov, Albert and Vaidman [AAV] which in turn played an important role in recent developments in quantum cosmology $[\mathrm{ST}]$.

Independently, and on a more pragmatic ground, new ideas have emerged in the study of nonequilibrium processes [Ru2]. Structured by the concepts of nonequilibrium steady state and entropy production, they have triggered intense activity in both theoretical and experimental physics. In the resulting theoretical framework, classical fluctuation relations [ECM, GC1, GC2, Jar, Cr1] hint at new links between the thermodynamic formalism of dynamical systems [Ru1] and statistical mechanics. In this formalism, the time arrow is intimately linked with information theoretic concepts and its emergence can be precisely quantified. Fluctuation relations have been extended to quantum dynamics [Ku2, Ta, EHM, CHT, DL, JOPP], allowing for the study of the arrow of time in open quantum systems [JOPS, BSS].

While repeated quantum measurement processes have recently received much focus [SVW, GPP, YK, $\mathrm{BB}, \mathrm{BBB}, \mathrm{BFFS}$ ], their connections with the above mentioned advances of nonequilibrium statistical mechanics have not been fully explored. This work is our first step in this direction of research. It aims at a better understanding of the large time asymptotics of the statistics of the fluctuations of entropy production in repeated quantum measurements. We shall in particular derive the large deviation principle for these fluctuations and prove the so-called fluctuation theorem. We shall also quantify the emergence of the arrow of time by linking hypothesis testing exponents distinguishing past and future to the large deviation principle for fluctuations of entropy production.

Finally, we stress that even though the systems of interest in this work are of a genuine quantum nature, the resulting sequences of measurement outcomes are described by classical dynamical systems. However, these dynamical systems do not generally satisfy the "chaotic hypothesis" of GallavottiCohen $^{2}$. From a mathematical perspective, the main results of this work are extensions of the thermodynamic formalism to this new class of dynamical systems.

\subsection{Setting}

We shall focus on measurements described by a (quantum) instrument $\mathcal{J}$ on a finite dimensional Hilbert space $\mathcal{H}$. We briefly recall the corresponding setup, referring the reader to [Ho, Section 5] for additional information and references to the literature, and to [Da, Section 4] for a discussion of pioneering works on the subject.

An instrument in the Heisenberg picture is a finite family $\mathcal{J}=\left\{\Phi_{a}\right\}_{a \in \mathcal{A}}$ of completely positive maps $\Phi_{a}: \mathcal{B}(\mathcal{H}) \rightarrow \mathcal{B}(\mathcal{H})^{3}$ such that $\Phi:=\sum_{a \in \mathcal{A}} \Phi_{a}$ satisfies $\Phi[\mathbb{1}]=\mathbb{1}$. The finite alphabet $\mathcal{A}$ describes

\footnotetext{
${ }^{2}$ The relevant invariant measures are non-Gibbsian.

${ }^{3} \mathcal{B}(\mathcal{H})$ denotes the algebra of all linear maps $X: \mathcal{H} \rightarrow \mathcal{H}$.
} 
the possible outcomes of a single measurement. We denote by $\mathcal{J}^{*}=\left\{\Phi_{a}^{*}\right\}_{a \in \mathcal{A}}$ the dual instrument in the Schrödinger picture, where $\Phi_{a}^{*}: \mathcal{B}(\mathcal{H}) \rightarrow \mathcal{B}(\mathcal{H})$ is defined by $\operatorname{tr}\left(\Phi_{a}^{*}[X] Y\right)=\operatorname{tr}\left(X \Phi_{a}[Y]\right)$.

A pair $(\mathcal{J}, \rho)$, where $\mathcal{J}$ is an instrument and $\rho$ a density matrix on $\mathcal{H}$, defines a repeated measurement process in the following way. At time $t=1$, when the system is in the state $\rho$, a measurement is performed. The outcome $a_{1} \in \mathcal{A}$ is observed with probability $\operatorname{tr}\left(\Phi_{a_{1}}^{*}[\rho]\right)$, and after the measurement the system is in the state

$$
\rho_{a_{1}}=\frac{\Phi_{a_{1}}^{*}[\rho]}{\operatorname{tr}\left(\Phi_{a_{1}}^{*}[\rho]\right)}
$$

A further measurement at time $t=2$ gives the outcome $a_{2}$ with probability

$$
\operatorname{tr}\left(\Phi_{a_{2}}^{*}\left[\rho_{a_{1}}\right]\right)=\frac{\operatorname{tr}\left(\left(\Phi_{a_{2}}^{*} \circ \Phi_{a_{1}}^{*}\right)[\rho]\right)}{\operatorname{tr}\left(\Phi_{a_{1}}^{*}[\rho]\right)}
$$

and the joint probability for the occurence of the sequence of outcomes $\left(a_{1}, a_{2}\right)$ is

$$
\operatorname{tr}\left(\left(\Phi_{a_{2}}^{*} \circ \Phi_{a_{1}}^{*}\right)[\rho]\right)=\operatorname{tr}\left(\rho\left(\Phi_{a_{1}} \circ \Phi_{a_{2}}\right)[\mathbb{1}]\right) .
$$

Continuing inductively, we deduce that the distribution of the sequence $\left(a_{1}, \ldots, a_{T}\right) \in \mathcal{A}^{T}$ of outcomes of $T$ successive measurements is given by

$$
\mathbb{P}_{T}\left(a_{1}, \ldots, a_{T}\right)=\operatorname{tr}\left(\rho\left(\Phi_{a_{1}} \circ \cdots \circ \Phi_{a_{T}}\right)[\mathbb{1}]\right) .
$$

One easily verifies that, due to the relation $\Phi[\mathbb{1}]=\mathbb{1}, \mathbb{P}_{T}$ is indeed a probability measure on $\mathcal{A}^{T}$.

Example 1. von Neumann measurements. Suppose that $\mathcal{A} \subset \mathbb{R}$. Let $\left\{P_{a}\right\}_{a \in \mathcal{A}}$ be a family of orthogonal projections satisfying $\sum_{a \in \mathcal{A}} P_{a}=\mathbb{1}$, and let the unitary $U: \mathcal{H} \rightarrow \mathcal{H}$ be the propagator of the system over a unit time interval. The instrument defined by $\Phi_{a}[X]=V_{a} X V_{a}^{*}$, where $V_{a}=U^{*} P_{a}$, describes the projective von Neumann measurement of the observable $A=\sum_{a \in \mathcal{A}} a P_{a}$. More precisely, if the system is in the state $\rho$ at time $t$, then a measurement of $A$ at time $t+1$ yields $a$ with the probability $\operatorname{tr}\left(\Phi_{a}^{*}[\rho]\right)$.

Example 2. Ancila measurements. Let $\mathcal{H}_{p}$ be a finite dimensional Hilbert space describing a "probe" allowed to interact with our system. The initial state of the probe is described by the density matrix $\rho_{p}$. The Hilbert space and the initial state of the joint system are $\mathcal{H} \otimes \mathcal{H}_{p}$ and $\rho \otimes \rho_{p}$. Let $U: \mathcal{H} \otimes \mathcal{H}_{p} \rightarrow \mathcal{H} \otimes \mathcal{H}_{p}$ be the unitary propagator of the joint system over a unit time interval. Let $\left\{P_{a}\right\}_{a \in \mathcal{A}}$ be a family of orthogonal projections on $\mathcal{H}_{p}$ such that $\sum_{a \in \mathcal{A}} P_{a}=\mathbb{1}$ and define

$$
\Phi_{a}^{*}[\rho]=\operatorname{tr}_{\mathcal{H}_{p}}\left(\left(\mathbb{1} \otimes P_{a}\right) U\left(\rho \otimes \rho_{p}\right) U^{*}\right),
$$

where $\operatorname{tr}_{\mathcal{H}_{p}}$ stands for the partial trace over $\mathcal{H}_{p}$. The maps $\Phi_{a}^{*}$ extend in an obvious way to linear maps $\Phi_{a}^{*}: \mathcal{B}(\mathcal{H}) \rightarrow \mathcal{B}(\mathcal{H})$, and the family $\left\{\Phi_{a}^{*}\right\}_{a \in \mathcal{A}}$ is an instrument on $\mathcal{H}$ in the Schrödinger picture. ${ }^{4}$ Moreover, any such instrument arises in this way: given $\left\{\Phi_{a}^{*}\right\}_{a \in \mathcal{A}}$, one can find $\mathcal{H}_{p}, \rho_{p}, U$, and $\left\{P_{a}\right\}_{a \in \mathcal{A}}$ so that (1.1) holds for all density matrices $\rho$ on $\mathcal{H}^{5}$

\footnotetext{
${ }^{4}$ The same instrument in the Heisenberg picture is described by $\Phi_{a}[X]=\operatorname{tr}_{\mathcal{H}_{p}}\left(U^{*}\left(X \otimes P_{a}\right) U\left(\mathbb{1} \otimes \rho_{p}\right)\right)$.

${ }^{5}$ In our setting, this result is an immediate consequence of Stinespring's dilation theorem. For generalizations, see [Ho, Section 5].
} 
On entropy production of repeated quantum measurements I

Example 3. Perfect instruments. An instrument $\mathcal{J}=\left\{\Phi_{a}\right\}_{a \in \mathcal{A}}$ is called perfect if, for all $a \in \mathcal{A}, \Phi_{a}[X]=V_{a} X V_{a}^{*}$ for some $V_{a} \in \mathcal{B}(\mathcal{H})$. The instruments associated to von Neumann measurements are perfect. The instrument of an ancila measurement is perfect if $\operatorname{dim} P_{a}=1$ for all $a \in \mathcal{A}$ and $\rho_{p}$ is a pure state, i.e., $\rho_{p}=\left|\psi_{p}\right\rangle\left\langle\psi_{p}\right|$ for some unit vector $\psi_{p} \in \mathcal{H}_{p}$.

Example 4. Unraveling of a quantum channel. Let $\Phi: \mathcal{B}(\mathcal{H}) \rightarrow \mathcal{B}(\mathcal{H})$ be a completely positive unital map - a quantum channel. Any such $\Phi$ has a (non-unique) Kraus representation

$$
\Phi[X]=\sum_{a \in \mathcal{A}} V_{a} X V_{a}^{*}
$$

where $\mathcal{A}$ is a finite set and the $V_{a} \in \mathcal{B}(\mathcal{H})$ are such that $\sum_{a \in \mathcal{A}} V_{a} V_{a}^{*}=\mathbb{1}$ (see, e.g., [Pe, Theorem 2.2]). The maps $\Phi_{a}[X]=V_{a} X V_{a}^{*}$ define a perfect instrument. The process $(\mathcal{J}, \rho)$ induced by the Kraus family $\mathcal{J}=\left\{\Phi_{a}\right\}_{a \in \mathcal{A}}$ and a state $\rho$ is a so-called unraveling of $\Phi$.

The property $\Phi[\mathbb{1}]=\mathbb{1}$ ensures that the family $\left\{\mathbb{P}_{T}\right\}_{T \geq 1}$ uniquely extends to a probability measure $\mathbb{P}$ on $(\Omega, \mathcal{F})$, where $\Omega=\mathcal{A}^{\mathbb{N}}$ and $\mathcal{F}$ is the $\sigma$-algebra on $\Omega$ generated by the cylinder sets. $\mathbb{E}[\cdot]$ denotes the expectation w.r.t. this measure. We equip $\Omega$ with the usual product topology. $\Omega$ is metrizable and a convenient metric for our purposes is $d\left(\omega, \omega^{\prime}\right)=\lambda^{k\left(\omega, \omega^{\prime}\right)}$, where $\left.\lambda \in\right] 0,1\left[\right.$ is fixed and $k\left(\omega, \omega^{\prime}\right)=$ $\inf \left\{j \mid \omega_{j} \neq \omega_{j}^{\prime}\right\}$; see [Ru1, Section 7.2]. $(\Omega, d)$ is a compact metric space and its Borel $\sigma$-field coincides with $\mathcal{F}$. For any integers $1 \leq i \leq j$ we set $\llbracket i, j \rrbracket=[i, j] \cap \mathbb{N}$ and denote $\Omega_{T}=\mathcal{A}^{\llbracket 1, T \rrbracket}$. The left shift

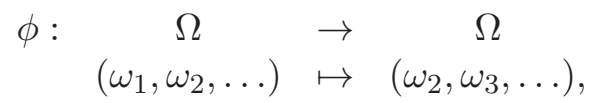

is a continuous surjection. If the initial state $\rho$ satisfies $\Phi^{*}[\rho]=\rho{ }^{6}$ then $\mathbb{P}$ is $\phi$-invariant (i.e., $\mathbb{P}\left(\phi^{-1}(A)\right)=\mathbb{P}(A)$ for all $\left.A \in \mathcal{F}\right)$ and the process $(\mathcal{J}, \rho)$ defines a dynamical system $(\Omega, \mathbb{P}, \phi)$. This observation leads to our first assumption:

Assumption (A) The initial state satisfies $\Phi^{*}[\rho]=\rho$ and $\rho>0$.

In what follows we shall always assume that (A) holds. Some of our results hold under a weaker form of Assumption (A); see Remark 2 after Theorem 2.3.

The basic ergodic properties of dynamical system $(\Omega, \mathbb{P}, \phi)$ can be characterized in terms of $\Phi$ as follows. Note that the spectral radius of $\Phi$ is 1 .

Theorem 1.1 (1) If 1 is a simple eigenvalue of $\Phi$, then $(\Omega, \mathbb{P}, \phi)$ is ergodic.

(2) If 1 is a simple eigenvalue of $\Phi$ and $\Phi$ has no other eigenvalues on the unit circle $|z|=1$, then $(\Omega, \mathbb{P}, \phi)$ is a $K$-system, and in particular it is mixing. Moreover, for any two Hölder continuous functions $f, g: \Omega \rightarrow \mathbb{R}$ there exists a constant $\gamma>0$ such that

$$
\mathbb{E}\left(f g \circ \phi^{n}\right)-\mathbb{E}(f) \mathbb{E}(g)=O\left(\mathrm{e}^{-\gamma n}\right) .
$$

\footnotetext{
${ }^{6}$ Since $\Phi[\mathbb{1}]=\mathbb{1}$, such a density matrix $\rho$ always exists.
} 
Remark 1. These results can be traced back to [FNW]; see Remark 1 in Section 1.3. Related results can be found in [KM1, KM2, MP]. For a pedagogical exposition of the proofs and additional information we refer the reader to [BJPP3].

Remark 2. 1 is a simple eigenvalue of $\Phi$ whenever $\Phi$ is irreducible, i.e., the relation $\Phi[P] \leq \lambda P$ for some orthogonal projection $P \in \mathcal{B}(\mathcal{H})$ and some $\lambda>0$ implies $P \in\{0, \mathbb{1}\}$; see [EHK, Lemma 4.1].

Remark 3. Note that the condition $\Phi^{*}[\rho]=\rho$ is equivalent to

$$
\sum_{\omega_{1}, \ldots, \omega_{S} \in \mathcal{A}} \mathbb{P}_{S+T}\left(\omega_{1}, \ldots, \omega_{S}, \omega_{S+1}, \ldots, \omega_{S+T}\right)=\mathbb{P}_{T}\left(\omega_{S+1}, \ldots, \omega_{S+T}\right),
$$

which is usually seen as an effect of decoherence. Under the Assumptions of Theorem 1.1 (2) the state $\rho$ satisfying Assumption (A) is unique. Moreover, for any density matrix $\rho_{0}$, one has

$$
\lim _{S \rightarrow \infty} \sum_{\omega_{1}, \ldots, \omega_{S} \in \mathcal{A}} \operatorname{tr}\left(\rho_{0} \Phi_{\omega_{1}} \circ \cdots \circ \Phi_{\omega_{S}} \circ \Phi_{\omega_{S+1}} \circ \cdots \circ \Phi_{\omega_{S+T}}\right)=\mathbb{P}_{T}\left(\omega_{S+1}, \ldots, \omega_{S+T}\right),
$$

i.e., the dynamical system $(\Omega, \mathbb{P}, \phi)$ describes the process $\left(\mathcal{J}, \rho_{0}\right)$ in the asymptotic regime where a long sequence of initial measurements is disregarded.

We proceed to describe the entropic aspects of the dynamical system $(\Omega, \mathbb{P}, \phi)$ generated by the repeated measurement process $(\mathcal{J}, \rho)$ that will be our main concern. Let $\theta: \mathcal{A} \rightarrow \mathcal{A}$ be an involution. For each $T \geq 1$ we define an involution on $\Omega_{T}$ by

$$
\Theta_{T}\left(\omega_{1}, \ldots, \omega_{T}\right)=\left(\theta\left(\omega_{T}\right), \ldots, \theta\left(\omega_{1}\right)\right) .
$$

A process $(\widehat{\mathcal{J}}, \widehat{\rho})$ is called an outcome reversal (abbreviated OR) of the process $(\mathcal{J}, \rho)$ whenever the instrument $\widehat{\mathcal{J}}=\left\{\widehat{\Phi}_{a}\right\}_{a \in \mathcal{A}}$ and the density matrix $\widehat{\rho}$ acting on the same Hilbert space $\mathcal{H}$ satisfy $\widehat{\Phi}^{*}[\widehat{\rho}]=\widehat{\rho}>0$, and the induced probability measures

$$
\widehat{\mathbb{P}}_{T}\left(\omega_{1}, \ldots, \omega_{T}\right)=\operatorname{tr}\left(\widehat{\rho}\left(\widehat{\Phi}_{\omega_{1}} \circ \cdots \circ \widehat{\Phi}_{\omega_{T}}\right)[\mathbb{1}]\right)
$$

satisfy

$$
\widehat{\mathbb{P}}_{T}=\mathbb{P}_{T} \circ \Theta_{T}
$$

for all $T \geq 1$. Such a process always exists and a canonical choice is

$$
\widehat{\Phi}_{a}(X)=\rho^{-\frac{1}{2}} \Phi_{\theta(a)}^{*}\left[\rho^{\frac{1}{2}} X \rho^{\frac{1}{2}}\right] \rho^{-\frac{1}{2}}, \quad \widehat{\rho}=\rho ;
$$

see [Cr2]. Indeed, one easily checks that $\left\{\widehat{\Phi}_{a}\right\}_{a \in \mathcal{A}}$ is an instrument such that $\widehat{\Phi}^{*}[\widehat{\rho}]=\widehat{\rho}$ and that (1.2) holds. Needless to say, the OR process $(\widehat{\mathcal{J}}, \widehat{\rho})$ need not be unique; see [BJPP2] for a discussion of this point. However, note that the family $\left\{\widehat{\mathbb{P}}_{T}\right\}_{T \geq 1}$ defined by (1.2) induces a unique $\phi$-invariant probability measure $\widehat{\mathbb{P}}$ on $\Omega$ : the OR dynamical system $(\Omega, \widehat{\mathbb{P}}, \phi)$ is completely determined by $(\Omega, \mathbb{P}, \phi)$ and the involution $\theta$.

In the present setting our study of the quantum arrow of time concerns the distinguishability between $(\mathcal{J}, \rho)$ and its $\mathrm{OR}(\widehat{\mathcal{J}}, \widehat{\rho})$ quantified by the entropic distinguishability of the respective probability measures $\mathbb{P}$ and $\widehat{\mathbb{P}}$. This entropic distinguishability is intimately linked with entropy production and hypothesis testing of the same pairs and we shall examine it on two levels:

Level I: Asymptotics of relative entropies and mean entropy production rate, Stein error exponent.

Level II: Asymptotics of Rényi's relative entropies and fluctuations of entropy production, large deviation principle and fluctuation theorem, Chernoff and Hoeffding error exponents. 
On entropy production of repeated quantum measurements I

\subsection{Two remarks}

Remark 1. The dynamical systems $(\Omega, \mathbb{P}, \phi)$ studied in this paper constitute a special class of $C^{*}$ finitely correlated states introduced in the seminal paper [FNW]. We recall the well-known construction. Let $\mathfrak{C}_{1}, \mathfrak{C}_{2}$ be two finite-dimensional $C^{*}$-algebras, $\rho$ a state on $\mathfrak{C}_{2}$, and $\mathcal{E}: \mathfrak{C}_{1} \otimes \mathfrak{C}_{2} \rightarrow \mathfrak{C}_{2}$ a completely positive unital map such that for all $B \in \mathfrak{C}_{2}$,

$$
\rho\left(\mathcal{E}\left(\mathbb{1}_{\mathfrak{C}_{1}} \otimes B\right)\right)=\rho(B) .
$$

For each $A \in \mathfrak{C}_{1}$ one defines a map $\mathcal{E}_{A}: \mathfrak{C}_{2} \rightarrow \mathfrak{C}_{2}$ by setting $\mathcal{E}_{A}(B)=\mathcal{E}(A \otimes B)$. The map

$$
\gamma_{n}\left(A_{1} \otimes \cdots \otimes A_{n}\right)=\rho\left(\mathcal{E}_{A_{1}} \cdots \mathcal{E}_{A_{n}}\left(\mathbb{1}_{\mathfrak{C}_{2}}\right)\right)
$$

uniquely extends to a state on the tensor product $\bigotimes_{i=1}^{n} \mathfrak{C}_{1}^{(i)}$, where $\mathfrak{C}_{1}^{(i)}$ is a copy of $\mathcal{C}_{1}$. Finally, the family of states $\gamma_{n}, n \in \mathbb{N}$, uniquely extends to a state $\gamma$ on the $C^{*}$-algebra $\bigotimes_{i \in \mathbb{N}} \mathfrak{C}_{1}^{(i)}$. The state $\gamma$ is the $C^{*}$-finitely correlated state associated to $\left(\mathfrak{C}_{1}^{*}, \mathfrak{C}_{2}, \mathcal{E}, \rho\right)$. The pairs $(\Omega, \mathbb{P})$ that arise in repeated quantum measurements of finite quantum systems correspond precisely to $C^{*}$-finitely correlated states with commutative $\mathfrak{C}_{1}$ and $\mathfrak{C}_{2}=\mathcal{B}(\mathcal{H})$ for some finite dimensional Hilbert space $\mathcal{H}$. This connection will play an important role in the continuation of this work [BJPP2].

In this context we also mention a pioneering work of Lindblad [Li] who studied the entropy of finitely correlated states generated by a non-Markovian adapted sequence of instruments. Since the main focus of this paper is the entropy production, the Lindblad work is only indirectly related to ours, and we will comment further on it in [BJPP2].

Remark 2. It is important to emphasize that the object of our study is the classical dynamical system $(\Omega, \mathbb{P}, \phi)$ and that the thermodynamic formalism of entropic fluctuations we will develop here is classical in nature. The quantum origin of the dynamical system $(\Omega, \mathbb{P}, \phi)$ manifests itself in the interpretation of our results and in the properties of the measure $\mathbb{P}$. The latter differ significantly from the ones usually assumed in the Gibbsian approach to the thermodynamic formalism. In particular, the Gibbsian theory of entropic fluctuations pioneered in [GC1, GC2] and further developed in [JPR, MV] cannot be applied to $(\Omega, \mathbb{P}, \phi)$, and a novel approach is needed. We will comment further on this point in Section 2.6. Here we mention only that the main technical tool of our work is the subadditive ergodic theory of dynamical systems developed in unrelated studies of the multifractal analysis of a certain class of self-similar sets; see [BaL, BV, CFH, CZC, Fa, FS, Fe1, Fe2, Fe3, FL, FK, IY, KW]. This tool sheds an unexpected light on the statistics of repeated quantum measurements.

\subsection{Organization}

The paper is organized as follows. Sections 2.1-2.3 deal with Level I: Asymptotics of relative entropies and mean entropy production rate, Stein error exponent. In Section 2.1, we fix our notation regarding various kinds of entropies that will appear in the paper. In Section 2.2 we state our results concerning the entropy production rate of the process $(\mathcal{J}, \rho)$. Stein's error exponents are discussed in Section 2.3. Sections 2.4-2.9 deal with Level II: Asymptotics of Rényi's relative entropies and fluctuations of entropy production, large deviation principle and fluctuation theorem, Chernoff and Hoeffding error exponents. Additional notational conventions and properties of entropies are discussed in Section 2.4. Section 2.5 is devoted to Rényi's relative entropy and its thermodynamic formalism. Fluctuations of the entropy production rate, including Large Deviation Principles as well as local and global Fluctuation Theorems are stated in Section 2.8. In Section 2.9 we discuss hypothesis testing 
and, in particular, the Chernoff and Hoeffding error exponents. The proofs are collected in Sections 3 and 4 .

For reasons of space, the discussion of concrete models of repeated quantum measurements to which our results apply will be presented in the continuation of this work [BJPP1].

Acknowledgments. We are grateful to Martin Fraas, Jürg Fröhlich and Daniel Ueltschi for useful discussions. The research of T.B. was partly supported by ANR project RMTQIT (Grant No. ANR-12-IS01-0001-01) and by ANR contract ANR-14-CE25-0003-0. The research of V.J. was partly supported by NSERC. Y.P. was partly supported by ANR contract ANR-14-CE25-0003-0. Y.P. also wishes to thank UMI-CRM for financial support and McGill University for its hospitality. The work of C.-A.P. has been carried out in the framework of the Labex Archimède (ANR-11-LABX-0033) and of the A*MIDEX project (ANR-11-IDEX-0001-02), funded by the "Investissements d'Avenir" French Government program managed by the French National Research Agency (ANR).

\section{Results}

\subsection{Level I: Entropies}

Let $\mathcal{X}$ be a finite set. We denote by $\mathcal{P}_{\mathcal{X}}$ the set of all probability measures on $\mathcal{X}$. The Gibbs-Shannon entropy $^{7}$ of $P \in \mathcal{P}_{\mathcal{X}}$ is

$$
S(P)=-\sum_{x \in \mathcal{X}} P(x) \log P(x) .
$$

The map $\mathcal{P}_{\mathcal{X}} \ni P \mapsto S(P)$ is continuous and takes values in $[0, \log |\chi|]$. The entropy is subadditive: if $\mathcal{X}=\mathcal{X}_{1} \times \mathcal{X}_{2}$ and $P_{1}, P_{2}$ are the respective marginals of $P \in \mathcal{P}_{\mathcal{X}}$, then

$$
S(P) \leq S\left(P_{1}\right)+S\left(P_{2}\right),
$$

with the equality iff $P=P_{1} \times P_{2}$. The entropy is also concave and almost convex: if $P_{k} \in \mathcal{P}_{\mathcal{X}}$ for $k=1, \ldots, n$ and $p_{k} \geq 0$ are such that $\sum_{k=1}^{n} p_{k}=1$, then

$$
\sum_{k=1}^{n} p_{k} S\left(P_{k}\right) \leq S\left(\sum_{k=1}^{n} p_{k} P_{k}\right) \leq S\left(p_{1}, \ldots, p_{n}\right)+\sum_{k=1}^{n} p_{k} S\left(P_{k}\right),
$$

where $S\left(p_{1}, \ldots, p_{n}\right)=-\sum_{k=1}^{n} p_{k} \log p_{k}$.

The set $\operatorname{supp} P=\{x \in \mathcal{X} \mid P(x) \neq 0\}$ is called the support of $P \in \mathcal{P}_{\mathcal{X}}$. For $\alpha \in \mathbb{R}$, the Rényi $\alpha$-entropy of $P$ is defined by

$$
S_{\alpha}(P)=\log \left[\sum_{x \in \operatorname{supp} P} P(x)^{\alpha}\right] .
$$

The map $\alpha \mapsto S_{\alpha}(P)$ is real analytic and convex. Obviously,

$$
\left.\frac{\mathrm{d}}{\mathrm{d} \alpha} S_{\alpha}(P)\right|_{\alpha=1}=-S(P)
$$

\footnotetext{
${ }^{7}$ In the sequel we will just refer to it as the entropy.
} 
The relative entropy of the pair $(P, Q) \in \mathcal{P}_{\mathcal{X}} \times \mathcal{P}_{\mathcal{X}}$ is

$$
S(P \mid Q)= \begin{cases}\sum_{x \in \mathcal{X}} P(x) \log \frac{P(x)}{Q(x)}, & \text { if } \operatorname{supp} P \subset \operatorname{supp} Q ; \\ +\infty, & \text { otherwise. }\end{cases}
$$

The map $(P, Q) \mapsto S(P \mid Q)$ is lower semicontinuous and jointly convex. One easily shows that $S(P \mid Q) \geq 0$ with equality iff $P=Q$. As a simple consequence, we note the log-sum inequality, ${ }^{8}$

$$
\sum_{j=1}^{M} a_{j} \log \frac{a_{j}}{b_{j}} \geq a \log \frac{a}{b}, \quad a=\sum_{j=1}^{M} a_{j}, \quad b=\sum_{j=1}^{M} b_{j},
$$

valid for non-negative $a_{j}, b_{j}$.

If $\mathcal{Y}$ is another finite set, a matrix $[M(x, y)]_{(x, y) \in \mathcal{X} \times \mathcal{Y}}$ with non-negative entries is called stochastic if for all $x \in \mathcal{X}, \sum_{y \in \mathcal{Y}} M(x, y)=1$. A stochastic matrix induces a transformation $M: \mathcal{P}_{\mathcal{X}} \rightarrow \mathcal{P}_{\mathcal{Y}}$ by

$$
M(P)(y)=\sum_{x \in \mathcal{X}} P(x) M(x, y)
$$

The relative entropy is monotone with respect to stochastic transformations:

$$
S(M(P) \mid M(Q)) \leq S(P \mid Q)
$$

For $\alpha \in \mathbb{R}$, the Rényi relative $\alpha$-entropy of a pair $(P, Q)$ satisfying $\operatorname{supp} P=\operatorname{supp} Q$ is defined by

$$
S_{\alpha}(P \mid Q)=\log \left[\sum_{x \in \mathcal{X}} P(x)^{1-\alpha} Q(x)^{\alpha}\right] .
$$

The map $\mathbb{R} \ni \alpha \mapsto S_{\alpha}(P \mid Q)$ is real analytic and convex. It clearly satisfies

$$
S_{\alpha}(P \mid Q)=S_{1-\alpha}(Q \mid P)
$$

and in particular $S_{0}(P \mid Q)=S_{1}(P \mid Q)=0$. Convexity further implies that $S_{\alpha}(P \mid Q) \leq 0$ for $\alpha \in[0,1]$ and $S_{\alpha}(P \mid Q) \geq 0$ for $\alpha \notin[0,1]$. The Renyi relative entropy relates to the relative entropy through

$$
\left.\frac{\mathrm{d}}{\mathrm{d} \alpha} S_{\alpha}(P \mid Q)\right|_{\alpha=0}=-S(P \mid Q),\left.\quad \frac{\mathrm{d}}{\mathrm{d} \alpha} S_{\alpha}(P \mid Q)\right|_{\alpha=1}=S(Q \mid P) .
$$

The map $(P, Q) \rightarrow S_{\alpha}(P \mid Q)$ is continuous. For $\alpha \in[0,1]$ this map is jointly concave and

$$
S_{\alpha}(P \mid Q) \leq S_{\alpha}(M(P) \mid M(Q))
$$

We note also that if $\varphi: \mathcal{X} \rightarrow \mathcal{X}$ is a bijection, then

$$
S(P \circ \varphi \mid Q \circ \varphi)=S(P \mid Q), \quad S_{\alpha}(P \circ \varphi \mid Q \circ \varphi)=S_{\alpha}(P \mid Q) .
$$

\footnotetext{
${ }^{8}$ We use the conventions $0 / 0=0$ and $x / 0=\infty$ for $x>0, \log 0=-\infty, \log \infty=\infty, 0 \cdot( \pm \infty)=0$.
} 
All the above entropies can be characterized by a suitable variant of the Gibbs variational principle. We note in particular that for any $P \in \mathcal{P}_{\mathcal{X}}$ and any function $f: \mathcal{X} \rightarrow \mathbb{R}$ one has

$$
F_{P}(f):=\log \left(\sum_{x \in \mathcal{X}} \mathrm{e}^{f(x)} P(x)\right)=\max _{Q \in \mathcal{P} \mathcal{X}}\left(\sum_{x \in \mathcal{X}} f(x) Q(x)-S(Q \mid P)\right),
$$

and that the maximum is achieved by the measure

$$
P_{f}(x)=\mathrm{e}^{f(x)-F_{P}(f)} P(x) .
$$

For further information about these fundamental notions we refer the reader to $[\mathrm{AD}, \mathrm{OP}]$.

\subsection{Level I: Entropy production rate}

It follows from Eq. (1.2) that supp $\mathbb{P}_{T}$ and supp $\widehat{\mathbb{P}}_{T}$ have the same cardinality. Thus, if either $\operatorname{supp} \mathbb{P}_{T} \subset \operatorname{supp} \widehat{\mathbb{P}}_{T}$ or supp $\widehat{\mathbb{P}}_{T} \subset \operatorname{supp} \mathbb{P}_{T}$, then supp $\mathbb{P}_{T}=\operatorname{supp} \widehat{\mathbb{P}}_{T}$.

Notation. In the sequel $\mathbb{P}_{T}^{\#}$ denotes either $\mathbb{P}_{T}$ or $\widehat{\mathbb{P}}_{T}$.

The relation

$$
\mathbb{P}_{T}^{\#}\left(\omega_{1}, \ldots, \omega_{T}\right)=\sum_{\omega_{T+1} \in \mathcal{A}} \mathbb{P}_{T+1}^{\#}\left(\omega_{1}, \ldots, \omega_{T}, \omega_{T+1}\right)
$$

further gives that if supp $\mathbb{P}_{T} \neq \operatorname{supp} \widehat{\mathbb{P}}_{T}$ for some $T$, then $\operatorname{supp} \mathbb{P}_{T^{\prime}} \neq \operatorname{supp} \widehat{\mathbb{P}}_{T^{\prime}}$ for all $T^{\prime}>T$.

Define the function

$$
\Omega_{T} \ni \omega \mapsto \sigma_{T}(\omega)=\sigma_{T}\left(\omega_{1}, \ldots, \omega_{T}\right)=\log \frac{\mathbb{P}_{T}\left(\omega_{1}, \ldots, \omega_{T}\right)}{\widehat{\mathbb{P}}_{T}\left(\omega_{1}, \ldots, \omega_{T}\right)} .
$$

Note that $\sigma_{T}$ takes value in $[-\infty, \infty]$ and satisfies $\sigma_{T} \circ \Theta_{T}=-\sigma_{T}$. The family of random variables $\left\{\sigma_{T}\right\}_{T \geq 1}$ quantifies the irreversibility, or equivalently, the entropy production of our measurement process. The notion of entropy production of dynamical systems goes back to seminal works [ECM, ES, GC1, GC2]; see [JPR, Ku1, LS, Ma1, Ma2, MN, MV, RM].

The expectation value of $\sigma_{T}$ w.r.t. $\mathbb{P}$ is well-defined and is equal to the relative entropy of the pair $\left(\mathbb{P}_{T}, \widehat{\mathbb{P}}_{T}\right)$. More precisely, one has

$$
\mathbb{E}\left[\sigma_{T}\right]=\sum_{\omega \in \Omega_{T}} \sigma_{T}(\omega) \mathbb{P}_{T}(\omega)=S\left(\mathbb{P}_{T} \mid \widehat{\mathbb{P}}_{T}\right)=S\left(\mathbb{P}_{T} \circ \Theta_{T} \mid \widehat{\mathbb{P}}_{T} \circ \Theta_{T}\right)=S\left(\widehat{\mathbb{P}}_{T} \mid \mathbb{P}_{T}\right) .
$$

The log-sum inequality (2.6) and Eq. (2.13) give the pointwise inequality

$$
\sum_{\omega_{T+1} \in \mathcal{A}} \mathbb{P}_{T+1}\left(\omega_{1}, \ldots, \omega_{T+1}\right) \log \frac{\mathbb{P}_{T+1}\left(\omega_{1}, \ldots, \omega_{T+1}\right)}{\widehat{\mathbb{P}}_{T+1}\left(\omega_{1}, \ldots, \omega_{T+1}\right)} \geq \mathbb{P}_{T}\left(\omega_{1}, \ldots, \omega_{T}\right) \log \frac{\mathbb{P}_{T}\left(\omega_{1}, \ldots, \omega_{T}\right)}{\widehat{\mathbb{P}}_{T}\left(\omega_{1}, \ldots, \omega_{T}\right)}
$$

and summing over all $\omega \in \Omega_{T}$ gives

$$
\mathbb{E}\left[\sigma_{T+1}\right] \geq \mathbb{E}\left[\sigma_{T}\right] \geq 0
$$


On entropy production of repeated quantum measurements I

Note that dividing the above pointwise inequality by $\mathbb{P}_{T}\left(\omega_{1}, \ldots, \omega_{T}\right)$ shows that $\sigma_{T}$ is a submartingale w.r.t. the natural filtration. The martingale approach to the statistics of repeated measurement processes has been used in several previous studies; see [KM1, KM2, BB, BBB] and references therein. In this work, however, we shall base our investigations on subadditive ergodic theory which provides another perspective on the subject.

The definition and the properties of the entropy production we have described so far are of course quite general, and are applicable to any $\phi$-invariant probability measure $\mathbb{Q}$ on $(\Omega, \mathcal{F})$ with $\mathbb{Q}_{T}$ being the marginal of $\mathbb{Q}$ on $\Omega_{T}$ and $\widehat{\mathbb{Q}}_{T}=\mathbb{Q}_{T} \circ \Theta_{T}$. The remaining results of the present and all results of next sections, however, rely critically on a subadditivity property of $\mathbb{P}$ described in Lemma 3.4.

Since, in view of (2.15), the cases where $\mathbb{E}\left[\sigma_{T}\right]=\infty$ for some $T$ are of little interest, in what follows we shall assume:

Assumption (B) $\operatorname{supp} \mathbb{P}_{T}=\operatorname{supp} \widehat{\mathbb{P}}_{T}$ for all $T \geq 1$.

We shall say that a positive map $\Psi: \mathcal{B}(\mathcal{H}) \rightarrow \mathcal{B}(\mathcal{H})$ is strictly positive, and write $\Psi>0$, whenever $\Psi[X]>0$ for all $X>0$. One easily sees that this condition is equivalent to $\Psi[\mathbb{1}]>0$. Under Assumption (A), a simple criterion for the validity of Assumption (B) is that $\Phi_{a}>0$ for all $a \in \mathcal{A}$. Indeed, these two conditions imply

$$
\left(\Phi_{\omega_{1}} \circ \cdots \circ \Phi_{\omega_{T}}\right)[\mathbb{1}]>0, \quad \rho^{-\frac{1}{2}}\left(\Phi_{\theta\left(\omega_{1}\right)} \circ \cdots \circ \Phi_{\theta\left(\omega_{T}\right)}\right)\left[\rho^{\frac{1}{2}} \mathbb{1} \rho^{\frac{1}{2}}\right] \rho^{-\frac{1}{2}}>0,
$$

for all $T \geq 1$ and all $\omega=\left(\omega_{1}, \ldots, \omega_{T}\right) \in \Omega_{T}$. It follows from the canonical construction (1.3) that $\operatorname{supp} \mathbb{P}_{T}=\operatorname{supp} \widehat{\mathbb{P}}_{T}=\Omega_{T}$ for all $T \geq 1$.

Theorem 2.1 (1) The (possibly infinite) limit

$$
\operatorname{ep}(\mathcal{J}, \rho):=\lim _{T \rightarrow \infty} \frac{1}{T} \mathbb{E}\left[\sigma_{T}\right]
$$

exists. We call it the mean entropy production rate of the repeated measurement process $(\mathcal{J}, \rho)$.

(2) One has

$$
\operatorname{ep}(\mathcal{J}, \rho)=\sup _{T \geq 1} \frac{1}{T}\left(\mathbb{E}\left[\sigma_{T}\right]+\log \min \operatorname{sp}(\rho)\right) \geq 0,
$$

where $\operatorname{sp}(\rho)$ denotes the spectrum of the initial state $\rho$.

(3) For $\mathbb{P}$-a.e. $\omega \in \Omega$, the limit

$$
\lim _{T \rightarrow \infty} \frac{1}{T} \sigma_{T}(\omega)=\bar{\sigma}(\omega)
$$

exists. The random variable $\bar{\sigma}$ satisfies $\bar{\sigma} \circ \phi=\bar{\sigma}$ and

$$
\mathbb{E}[\bar{\sigma}]=\operatorname{ep}(\mathcal{J}, \rho) .
$$

Its negative part $\bar{\sigma}_{-}=\frac{1}{2}(|\bar{\sigma}|-\bar{\sigma})$ satisfies $\mathbb{E}\left[\bar{\sigma}_{-}\right]<\infty$. Moreover, if $\operatorname{ep}(\mathcal{J}, \rho)<\infty$, then

$$
\lim _{T \rightarrow \infty} \mathbb{E}\left[\left|\frac{1}{T} \sigma_{T}-\bar{\sigma}\right|\right]=0 .
$$

The number $\bar{\sigma}(\omega)$ is the entropy production rate of the process $(\mathcal{J}, \rho)$ along the trajectory $\omega$. 
Remark 1. If $\mathbb{P}$ is $\phi$-ergodic, then obviously $\bar{\sigma}(\omega)=\operatorname{ep}(\mathcal{J}, \rho)$ for $\mathbb{P}$-a.e. $\omega \in \Omega$.

Remark 2. Since $\sigma_{T} \circ \Theta_{T}=-\sigma_{T}$, Part (1) implies

$$
\lim _{T \rightarrow \infty} \frac{1}{T} \widehat{\mathbb{E}}\left[\sigma_{T}\right]=\lim _{T \rightarrow \infty} \frac{1}{T} \mathbb{E}\left[-\sigma_{T}\right]=-\operatorname{ep}(\mathcal{J}, \rho) .
$$

Part (3) applied to the OR dynamical system $(\Omega, \widehat{\mathbb{P}}, \phi)$ yields that the limit $(2.16)$ exists $\widehat{\mathbb{P}}$-a.e. and satisfies $\widehat{\mathbb{E}}[\bar{\sigma}]=-\operatorname{ep}(\mathcal{J}, \rho)$. Assuming that $\mathbb{P}$ is $\phi$-ergodic, we have either $\mathbb{P}=\widehat{\mathbb{P}}$ and hence $\operatorname{ep}(\mathcal{J}, \rho)=0$, or $\mathbb{P} \perp \widehat{\mathbb{P}}$ (i.e., $\mathbb{P}$ and $\widehat{\mathbb{P}}$ are mutually singular).

Remark 3. The assumption $\operatorname{ep}(\mathcal{J}, \rho)<\infty$ in Part (3) will be essential for most of the forthcoming results. It is ensured if $\Phi_{a}>0$ for all $a \in \mathcal{A}$. Indeed, the latter condition implies that $\Phi_{a}[\mathbb{1}] \geq \epsilon \mathbb{1}$ for some $\epsilon>0$ and all $a \in \mathcal{A}$. Since

$$
\begin{aligned}
\mathbb{E}\left[\sigma_{T}\right] \leq \mathbb{E}\left[-\log \widehat{\mathbb{P}}_{T}\right] & =-\sum_{\omega \in \Omega_{T}} \mathbb{P}_{T}(\omega) \log \widehat{\mathbb{P}}_{T}(\omega)=-\sum_{\omega \in \Omega_{T}} \widehat{\mathbb{P}}_{T}(\omega) \log \mathbb{P}_{T}(\omega) \\
& =-\sum_{\omega \in \Omega_{T}} \widehat{\mathbb{P}}_{T}(\omega) \log \operatorname{tr}\left(\rho\left(\Phi_{\omega_{1}} \circ \cdots \circ \Phi_{\omega_{T}}\right)[\mathbb{1}]\right),
\end{aligned}
$$

it follows that in this case $\operatorname{ep}(\mathcal{J}, \rho) \leq-\log \epsilon$. For a perfect instrument $\Phi_{a}[X]=V_{a} X V_{a}^{*}, \Phi_{a}[\mathbb{1}] \geq \epsilon \mathbb{1}$ for some $\epsilon>0$ and all $a \in \mathcal{A}$ iff all $V_{a}$ 's are invertible.

Remark 4. For $i=1,2, \ldots$, let $\left(\mathcal{J}_{i}, \rho_{i}\right)$ denote processes on a Hilbert space $\mathcal{H}_{i}$ with instrument $\mathcal{J}_{i}=\left\{\Phi_{i, a}\right\}_{a \in \mathcal{A}_{i}}$. Set $\Phi_{i}=\sum_{a \in \mathcal{A}_{i}} \Phi_{i, a}$ and assume that OR processes $\left(\widehat{\mathcal{J}}_{i}, \widehat{\rho}_{i}\right)$ are induced by involutions $\theta_{i}$ on $\mathcal{A}_{i}$. Denote by $\mathbb{P}_{i, T}^{\#}$ the probabilities induced on $\mathcal{A}_{i}^{T}$ by these processes. Basic operations on instruments and the resulting measurement processes have the following effects on entropy production.

Product. The process $\left(\mathcal{J}_{1} \otimes \mathcal{J}_{2}, \rho_{1} \otimes \rho_{2}\right)$ is defined on the Hilbert space $\mathcal{H}_{1} \otimes \mathcal{H}_{2}$ by the instrument $\mathcal{J}_{1} \otimes \mathcal{J}_{2}:=\left\{\Phi_{1, a_{1}} \otimes \Phi_{2, a_{2}}\right\}_{\left(a_{1}, a_{2}\right) \in \mathcal{A}_{1} \times \mathcal{A}_{2}}$ and its OR is induced by the involution $\theta\left(a_{1}, a_{2}\right)=$ $\left(\theta_{1}\left(a_{1}\right), \theta_{2}\left(a_{2}\right)\right)$. The probabilities induced on $\left(\mathcal{A}_{1} \times \mathcal{A}_{2}\right)^{T}$ by these processes are easily seen to be $\mathbb{P}_{T}^{\#}\left(\left(a_{1}, b_{1}\right), \ldots,\left(a_{T}, b_{T}\right)\right)=\mathbb{P}_{1, T}^{\#}\left(a_{1}, \ldots, a_{T}\right) \mathbb{P}_{2, T}^{\#}\left(b_{1}, \ldots, b_{T}\right)$ and it follows from the equality in Relation (2.4) and Eq. (2.14) that

$$
\operatorname{ep}\left(\mathcal{J}_{1} \otimes \mathcal{J}_{2}, \rho_{1} \otimes \rho_{2}\right)=\operatorname{ep}\left(\mathcal{J}_{1}, \rho_{1}\right)+\operatorname{ep}\left(\mathcal{J}_{2}, \rho_{2}\right) .
$$

Sums. The process $\left(\mathcal{J}_{1} \oplus \mathcal{J}_{2}, \rho^{(\mu)}\right)$ is defined on the Hilbert space $\mathcal{H}_{1} \oplus \mathcal{H}_{2}$ with the initial state $\left.\rho^{(\mu)}=\mu \rho_{1} \oplus(1-\mu) \rho_{2}, \mu \in\right] 0,1\left[\right.$, and the instrument $\mathcal{J}_{1} \oplus \mathcal{J}_{2}=\left\{\Phi_{a}\right\}_{a \in \mathcal{A}_{1} \cup \mathcal{A}_{2}}$ with

$$
\Phi_{a}[A]=\bigoplus_{i \in\{1,2\}} 1_{\mathcal{A}_{i}}(a) J_{i} \Phi_{i, a}\left[J_{i}^{*} A J_{i}\right] J_{i}^{*}
$$

where $1_{\mathcal{A}_{i}}$ denotes the characteristic function of $\mathcal{A}_{i}$ and $J_{i}$ the natural injection $\mathcal{H}_{i} \hookrightarrow \mathcal{H}_{1} \oplus \mathcal{H}_{2}$. It follows that

$$
\Phi[A]=\bigoplus_{i \in\{1,2\}} J_{i} \Phi_{i}\left[J_{i}^{*} A J_{i}\right] J_{i}^{*},
$$

and in particular $\Phi[\mathbb{1}]=\mathbb{1}$ and $\Phi^{*}\left[\rho^{(\mu)}\right]=\rho^{(\mu)}$. Assuming that $\theta_{1}$ and $\theta_{2}$ coincide on $\mathcal{A}_{1} \cap \mathcal{A}_{2}$, an OR process is induced by the involution defined on $\mathcal{A}_{1} \cup \mathcal{A}_{2}$ by $\theta(a)=\theta_{i}(a)$ for $a \in \mathcal{A}_{i}$. The probabilities induced by these processes are the convex combinations

$$
\mathbb{P}_{T}^{\#}=\mu \mathbb{P}_{1, T}^{\#}+(1-\mu) \mathbb{P}_{2, T}^{\#}
$$


On entropy production of repeated quantum measurements I

where $\mathbb{P}_{i, T}^{\#}$ is interpreted as a probability on $\mathcal{A}_{1} \cup \mathcal{A}_{2}$. The joint convexity of relative entropy and Relation (2.14) yield the inequality

$$
\operatorname{ep}\left(\mathcal{J}_{1} \oplus \mathcal{J}_{2}, \rho^{(\mu)}\right) \leq \mu \operatorname{ep}\left(\mathcal{J}_{1}, \rho_{1}\right)+(1-\mu) \operatorname{ep}\left(\mathcal{J}_{2}, \rho_{2}\right) .
$$

Note that if $\mathbb{P}_{1} \neq \mathbb{P}_{2}$, then the sum of two measurement processes is never ergodic. Two extreme cases are worth noticing: ${ }^{9}$

(a) If $\mathcal{A}_{1} \cap \mathcal{A}_{2}=\emptyset$ (disjoint sum), then $\mathbb{P}_{1, T} \perp \mathbb{P}_{2, T}$ and $\mathbb{P}_{T}\left(a_{1}, \ldots, a_{T}\right)=\mu_{i} \mathbb{P}_{i, T}\left(a_{1}, \ldots, a_{T}\right)$ $\left(\mu_{1}=\mu, \mu_{2}=1-\mu\right.$ ) for all $T \geq 1$ provided $a_{1} \in \mathcal{A}_{i}$, i.e., the outcome of the first measurement selects the distribution of the full history. It immediately follows that in this case

$$
\operatorname{ep}\left(\mathcal{J}_{1} \oplus \mathcal{J}_{2}, \rho^{(\mu)}\right)=\mu \operatorname{ep}\left(\mathcal{J}_{1}, \rho_{1}\right)+(1-\mu) \operatorname{ep}\left(\mathcal{J}_{2}, \rho_{2}\right) .
$$

(b) If $\mathcal{A}_{1}=\mathcal{A}_{2}=\mathcal{A}$ then $\mathbb{P}_{1, T}$ and $\mathbb{P}_{2, T}$ can be equivalent for all $T \geq 1$. However, this does not preclude that $\mathbb{P}_{1} \perp \mathbb{P}_{2}$. This is indeed the case if $\mathbb{P}_{1}$ and $\mathbb{P}_{2}$ are ergodic and distinct. Then, there exists two $\phi$-invariant subsets $\mathcal{O}_{i} \subset \mathcal{A}^{\mathbb{N}}$ such that $\mathbb{P}_{i}\left(\mathcal{O}_{j}\right)=\delta_{i j}, \mathbb{P}\left(\mathcal{O}_{1} \cup \mathcal{O}_{2}\right)=1$, and

$$
\lim _{T \rightarrow \infty} \frac{1}{T} \sum_{t=0}^{T-1} f \circ \phi^{t}(\omega)=\mathbb{E}_{i}[f],
$$

for $\mathbb{P}$-a.e. $\omega \in \mathcal{O}_{i}$ and all $f \in L^{1}\left(\mathcal{A}^{\mathbb{N}}, \mathrm{d} \mathbb{P}\right)$. Thus, in this case, the selection occurs asymptotically as $T \rightarrow \infty$.

The above operations and results extend in an obvious way to finitely many processes.

Coarse graining. We shall say that $\mathcal{J}_{2}$ is coarser than $\mathcal{J}_{1}$ and write $\mathcal{J}_{2} \succ \mathcal{J}_{1}$ whenever $\mathcal{H}_{1}=\mathcal{H}_{2}$ and there exists a stochastic matrix $\left[M_{a_{1} a_{2}}\right]_{\left(a_{1}, a_{2}\right) \in \mathcal{A}_{1} \times \mathcal{A}_{2}}$ such that

$$
\Phi_{2, a_{2}}=\sum_{a_{1} \in \mathcal{A}_{1}} M_{a_{1} a_{2}} \Phi_{1, a_{1}}
$$

for all $a_{2} \in \mathcal{A}_{2}$. Note that in this case $\Phi_{1}=\Phi_{2}$. In particular, $\left(\mathcal{J}_{2}, \rho\right)$ satisfies Assumption (A) iff $\left(\mathcal{J}_{1}, \rho\right)$ does. If $M_{\theta_{1}\left(a_{1}\right) \theta_{2}\left(a_{2}\right)}=M_{a_{1} a_{2}}$ for all $\left(a_{1}, a_{2}\right) \in \mathcal{A}_{1} \times \mathcal{A}_{2}$ then $\mathcal{J}_{2} \succ \mathcal{J}_{1}$ is equivalent to $\widehat{\mathcal{J}}_{2} \succ \widehat{\mathcal{J}}_{1}$ and the induced probability distributions are related by

$$
\mathbb{P}_{2, T}^{\#}=M_{T}\left(\mathbb{P}_{1, T}^{\#}\right)
$$

where $\left[M_{T, a b}\right]_{(a, b) \in \mathcal{A}_{1}^{T} \times \mathcal{A}_{2}^{T}}$ is the stochastic matrix defined by

$$
M_{T, a b}=\prod_{i=1}^{T} M_{a_{i} b_{i}} .
$$

It follows from Inequality (2.7) and Relation (2.14) that

$$
\operatorname{ep}\left(\mathcal{J}_{2}, \rho\right) \leq \operatorname{ep}\left(\mathcal{J}_{1}, \rho\right)
$$

\footnotetext{
${ }^{9}$ The alphabets $\mathcal{A}_{i}$ are immaterial and only serve the purpose of labeling individual measurements $\Phi_{i, a}$, thus the identification of elements of $\mathcal{A}_{1}$ and $\mathcal{A}_{2}$ in $\mathcal{A}_{1} \cap \mathcal{A}_{2}$ is purely conventional. We note, however, that this identification affects our definition of the sum of two instruments.
} 
Compositions. Assuming that $\mathcal{H}_{1}=\mathcal{H}_{2}=\mathcal{H}, \rho_{1}=\rho_{2}=\rho$, and $\Phi_{1, a_{1}} \circ \Phi_{2}=\Phi_{2} \circ \Phi_{1, a_{1}}$ for all $a_{1} \in \mathcal{A}_{1}$, the composition $\left(\mathcal{J}_{1} \circ \mathcal{J}_{2}, \rho\right)$ is the process with the alphabet $\mathcal{A}_{1} \times \mathcal{A}_{2}$, involution $\theta\left(a_{1}, a_{2}\right)=$ $\left(\theta_{1}\left(a_{1}\right), \theta_{2}\left(a_{2}\right)\right)$ and instrument $\mathcal{J}_{1} \circ \mathcal{J}_{2}=\left\{\Phi_{1, a_{1}} \circ \Phi_{2, a_{2}}\right\}_{\left(a_{1}, a_{2}\right) \in \mathcal{A}_{1} \times \mathcal{A}_{2}}$. Setting $M_{\left(a_{1}, a_{2}\right) b}=\delta_{a_{1}, b}$ one easily sees that $\widetilde{\mathcal{J}}_{1} \succ \mathcal{J}_{1} \circ \mathcal{J}_{2}$ where $\widetilde{\mathcal{J}}_{1}=\left\{\Phi_{1, a} \circ \Phi_{2}\right\}_{a \in \mathcal{A}_{1}}$. Due to our commutation assumption, the probabilities induced by $\widetilde{\mathcal{J}}_{1}$ coincide with that of $\mathcal{J}_{1}$. It follows that

$$
\operatorname{ep}\left(\mathcal{J}_{1}, \rho\right) \leq \operatorname{ep}\left(\mathcal{J}_{1} \circ \mathcal{J}_{2}, \rho\right)
$$

Limits. Let $\left(\mathcal{J}_{n}, \rho_{n}\right), n \in \mathbb{N}$, and $(\mathcal{J}, \rho)$ be processes with the same Hilbert space $\mathcal{H}$, alphabet $\mathcal{A}$ and involution $\theta$. We say that $\lim \left(\mathcal{J}_{n}, \rho_{n}\right)=(\mathcal{J}, \rho)$ if

$$
\lim _{n \rightarrow \infty}\left(\left\|\rho_{n}-\rho\right\|+\sum_{a \in \mathcal{A}}\left\|\Phi_{n, a}-\Phi_{a}\right\|\right)=0 .
$$

Part (2) of Theorem 2.1 gives

$$
\operatorname{ep}\left(\mathcal{J}_{n}, \rho_{n}\right)=\sup _{T \geq 1} \frac{S\left(\mathbb{P}_{n, T} \mid \widehat{\mathbb{P}}_{n, T}\right)+\log \lambda_{n}}{T},
$$

where $\lambda_{n}=\min \operatorname{sp}\left(\rho_{n}\right)$. This relation and the lower semicontinuity of the relative entropy give that for any $T$,

$$
\liminf _{n \rightarrow \infty} \operatorname{ep}\left(\mathcal{J}_{n}, \rho_{n}\right) \geq \liminf _{n \rightarrow \infty} \frac{S\left(\mathbb{P}_{n, T} \mid \widehat{\mathbb{P}}_{n, T}\right)+\log \lambda_{n}}{T} \geq \frac{S\left(\mathbb{P}_{T} \mid \widehat{\mathbb{P}}_{T}\right)+\log \lambda}{T},
$$

where $\lambda=\min \operatorname{sp}(\rho)$. We used that the convergence (2.17) implies $\lim _{n \rightarrow \infty} \widehat{\mathbb{P}}_{n, T}^{\#}=\widehat{\mathbb{P}}_{T}^{\#}$ and $\lim _{n \rightarrow \infty} \lambda_{n}=\lambda$. Since (2.18) also holds for $(\mathcal{J}, \rho)$ and $\left(\mathbb{P}_{T}, \widehat{\mathbb{P}}_{T}\right)$, we derive

$$
\liminf _{n \rightarrow \infty} \operatorname{ep}\left(\mathcal{J}_{n}, \rho_{n}\right) \geq \operatorname{ep}(\mathcal{J}, \rho) .
$$

The final result of this section deals with the vanishing of the entropy production.

Proposition 2.2 If $\mathbb{P}=\widehat{\mathbb{P}}$, then $\operatorname{ep}(\mathcal{J}, \rho)=0$. Reciprocally, in cases where $\operatorname{ep}(\mathcal{J}, \rho)=0$ the following hold:

(1) $\lim _{T \rightarrow \infty} \mathbb{E}\left[\sigma_{T}\right]=\sup _{T \geq 1} \mathbb{E}\left[\sigma_{T}\right]<\infty$.

(2) The measures $\mathbb{P}$ and $\widehat{\mathbb{P}}$ are mutually absolutely continuous with finite relative entropy.

(3) If $\mathbb{P}$ is $\phi$-ergodic, then $\mathbb{P}=\widehat{\mathbb{P}}$.

\subsection{Level I: Stein's error exponents}

For $\epsilon \in] 0,1[$ and $T \geq 1$ set

$$
s_{T}(\epsilon):=\min \left\{\widehat{\mathbb{P}}_{T}(\mathcal{T}) \mid \mathcal{T} \subset \Omega_{T}, \mathbb{P}_{T}\left(\mathcal{T}^{c}\right) \leq \epsilon\right\}
$$


On entropy production of repeated quantum measurements I

where $\mathcal{T}^{c}=\Omega_{T} \backslash \mathcal{T}$. Since $\widehat{\mathbb{P}}_{T}=\mathbb{P}_{T} \circ \Theta_{T}$, the right hand side of this expression is invariant under the exchange of $\mathbb{P}_{T}$ and $\widehat{\mathbb{P}}_{T}$. The Stein error exponents of the pair $(\mathbb{P}, \widehat{\mathbb{P}})$ are defined by

$$
\underline{s}(\epsilon)=\liminf _{T \rightarrow \infty} \frac{1}{T} \log s_{T}(\epsilon), \quad \bar{s}(\epsilon)=\limsup _{T \rightarrow \infty} \frac{1}{T} \log s_{T}(\epsilon) .
$$

In Section 2.9 we shall interpret these error exponents in the context of hypothesis testing of the arrow of time.

Theorem 2.3 Suppose that $\mathbb{P}$ is $\phi$-ergodic. Then, for all $\epsilon \in] 0,1$,

$$
\underline{s}(\epsilon)=\bar{s}(\epsilon)=-\operatorname{ep}(\mathcal{J}, \rho) .
$$

We finish with two remarks.

Remark 1. Theorem 2.3 and its proof give that

$$
\begin{aligned}
& \underline{s}=\inf \left\{\liminf _{T \rightarrow \infty} \frac{1}{T} \log \widehat{\mathbb{P}}_{T}\left(\mathcal{T}_{T}\right) \mid \mathcal{T}_{T} \subset \Omega_{T} \text { for } T \geq 1, \text { and } \lim _{T \rightarrow \infty} \mathbb{P}_{T}\left(\mathcal{T}_{T}^{c}\right)=0\right\}, \\
& \bar{s}=\inf \left\{\limsup _{T \rightarrow \infty} \frac{1}{T} \log \widehat{\mathbb{P}}_{T}\left(\mathcal{T}_{T}\right) \mid \mathcal{T}_{T} \subset \Omega_{T} \text { for } T \geq 1, \text { and } \lim _{T \rightarrow \infty} \mathbb{P}_{T}\left(\mathcal{T}_{T}^{c}\right)=0\right\},
\end{aligned}
$$

satisfy $\bar{s}=\underline{s}=-\operatorname{ep}(\mathcal{J}, \rho)$; see the final remark in Section 3.3.

Remark 2. Theorems 2.1 and 2.3 also hold whenever Assumption (A) is replaced by the following two conditions:

(a) There exists a density matrix $\rho_{\text {inv }}>0$ such that $\Phi^{*}\left[\rho_{\text {inv }}\right]=\rho_{\text {inv }}$.

(b) $\rho>0$.

In this case, $\operatorname{ep}(\mathcal{J}, \rho)$ does not depend on the choice of $\rho$, i.e., $\operatorname{ep}(\mathcal{J}, \rho)=\operatorname{ep}\left(\mathcal{J}, \rho_{\text {inv }}\right)$ for all density matrices $\rho>0$. Note however that if $\Phi^{*}[\rho] \neq \rho$, then, except in trivial cases, $\mathbb{P}$ is not $\phi$-invariant and the family $\left\{\widehat{\mathbb{P}}_{T}\right\}_{T \geq 1}$ does not define a probability measure on $\Omega$.

\subsection{Level II: Entropies}

We denote by $\mathcal{P}$ the set of all probability measures on $(\Omega, \mathcal{F})$ and by $\mathcal{P}_{\phi} \subset \mathcal{P}$ the subset of all $\phi$ invariant elements of $\mathcal{P}$. We endow $\mathcal{P}$ with the topology of weak convergence which coincides with the relative topology inherited from the weak-* topology of the dual of the Banach space $C(\Omega)$ of continuous functions on $\Omega$. This topology is metrizable and makes $\mathcal{P}$ a compact metric space and $\mathcal{P}_{\phi}$ a closed convex subset of $\mathcal{P}$. Moreover, $\mathcal{P}_{\phi}$ is a Choquet simplex whose extreme points are the $\phi$-ergodic probability measures on $\Omega$; see [Ru1, Section A.5.6].

For $\mathbb{Q} \in \mathcal{P}, \mathbb{Q}_{T}$ denotes the marginal of $\mathbb{Q}$ on $\Omega_{T}$. Reciprocally, a sequence $\left\{\mathbb{Q}_{T}\right\}_{T \geq 1}$, with $\mathbb{Q}_{T} \in$ $\mathcal{P}_{\Omega_{T}}$ defines a unique $\mathbb{Q} \in \mathcal{P}$ iff

$$
\sum_{\omega_{T} \in \mathcal{A}} \mathbb{Q}_{T}\left(\omega_{1}, \ldots, \omega_{T}\right)=\mathbb{Q}_{T-1}\left(\omega_{1}, \ldots, \omega_{T-1}\right)
$$


for all $T>1$. Moreover, $\mathbb{Q} \in \mathcal{P}_{\phi}$ iff, in addition,

$$
\sum_{\omega_{1} \in \mathcal{A}} \mathbb{Q}_{T}\left(\omega_{1}, \ldots, \omega_{T}\right)=\mathbb{Q}_{T-1}\left(\omega_{2}, \ldots, \omega_{T}\right)
$$

for all $T>1$. It follows that to each $\mathbb{Q} \in \mathcal{P}_{\phi}$ we can associate a time-reversed measure $\widehat{\mathbb{Q}} \in \mathcal{P}_{\phi}$ with the marginals $\widehat{\mathbb{Q}}_{T}=\mathbb{Q}_{T} \circ \Theta_{T}$. The map $\mathbb{Q} \mapsto \widehat{\mathbb{Q}}$ defines an affine involution $\Theta$ of $\mathcal{P}_{\phi}$. Clearly, the map $\Theta$ preserves the set of $\phi$-ergodic probability measures. In the following we associate to $\mathbb{Q} \in \mathcal{P}_{\phi}$ the family of signed measures on $\Omega$ defined by

$$
\mathbb{Q}^{(\alpha)}:=(1-\alpha) \mathbb{Q}+\alpha \widehat{\mathbb{Q}} .
$$

Note that $\widehat{\mathbb{Q}}^{(\alpha)}=\mathbb{Q}^{(1-\alpha)}$ and that $\mathbb{Q}^{(\alpha)} \in \mathcal{P}_{\phi}$ for $\alpha \in[0,1]$.

If $\mathbb{Q} \in \mathcal{P}_{\phi}$, then the subadditivity (2.4) of entropy gives $S\left(\mathbb{Q}_{T+T^{\prime}}\right) \leq S\left(\mathbb{Q}_{T}\right)+S\left(\mathbb{Q}_{T^{\prime}}\right)$ for all $T, T^{\prime} \geq 1$, and Fekete's Lemma (Lemma 3.1 below) yields that

$$
h_{\phi}(\mathbb{Q})=\lim _{T \rightarrow \infty} \frac{1}{T} S\left(\mathbb{Q}_{T}\right)=\inf _{T \geq 1} \frac{1}{T} S\left(\mathbb{Q}_{T}\right) .
$$

By the Kolmogorov-Sinai theorem [Wa, Theorem 4.18], the number $h_{\phi}(\mathbb{Q})$ is the Kolmogorov-Sinai entropy of the left shift $\phi$ w.r.t. the probability measure $\mathbb{Q} \in \mathcal{P}_{\phi}$ and lies in the interval $[0, \log \ell]$, where $\ell$ is the number of elements of the alphabet $\mathcal{A}$. The map $\mathcal{P}_{\phi} \ni \mathbb{Q} \mapsto h_{\phi}(\mathbb{Q})$ is upper semicontinuous. It is also affine (recall the concavity/convexity bound (2.5)), i.e.,

$$
h_{\phi}\left(\lambda \mathbb{Q}_{1}+(1-\lambda) \mathbb{Q}_{2}\right)=\lambda h_{\phi}\left(\mathbb{Q}_{1}\right)+(1-\lambda) h_{\phi}\left(\mathbb{Q}_{2}\right)
$$

for all $\mathbb{Q}_{1}, \mathbb{Q}_{2} \in \mathcal{P}_{\phi}$ and $\lambda \in[0,1]$. Moreover, since $S\left(\mathbb{Q}_{T}\right)=S\left(\widehat{\mathbb{Q}}_{T}\right)$, one has $h_{\phi}\left(\mathbb{Q}^{(\alpha)}\right)=h_{\phi}(\mathbb{Q})$ for all $\alpha \in[0,1]$.

For $\mathbb{Q}_{1}, \mathbb{Q}_{2} \in \mathcal{P}$, we write $\mathbb{Q}_{1} \ll \mathbb{Q}_{2}$ whenever $\mathbb{Q}_{1}$ is absolutely continuous w.r.t. $\mathbb{Q}_{2}$. In this case, $\mathrm{d} \mathbb{Q}_{1} / \mathrm{d} \mathbb{Q}_{2}$ denotes the Radon-Nikodym derivative of $\mathbb{Q}_{1}$ w.r.t. $\mathbb{Q}_{2}$. The relative entropy of the pair $\left(\mathbb{Q}_{1}, \mathbb{Q}_{2}\right)$ is

$$
S\left(\mathbb{Q}_{1} \mid \mathbb{Q}_{2}\right)= \begin{cases}\int \log \left(\frac{\mathrm{d} \mathbb{Q}_{1}}{\mathrm{~d} \mathbb{Q}_{2}}\right) \mathrm{d} \mathbb{Q}_{1}, & \text { if } \mathbb{Q}_{1} \ll \mathbb{Q}_{2} \\ +\infty, & \text { otherwise. }\end{cases}
$$

The map $\mathcal{P} \times \mathcal{P} \ni\left(\mathbb{Q}_{1}, \mathbb{Q}_{2}\right) \mapsto S\left(\mathbb{Q}_{1} \mid \mathbb{Q}_{2}\right)$ is lower semicontinuous. Moreover $S\left(\mathbb{Q}_{1} \mid \mathbb{Q}_{2}\right) \geq 0$ with equality iff $\mathbb{Q}_{1}=\mathbb{Q}_{2}$. The proofs of these basic facts can be found in [El]. If $f$ is a measurable function on $(\Omega, \mathcal{F})$, we shall denote its expectation w.r.t. $\mathbb{Q} \in \mathcal{P}$ by

$$
\mathbb{Q}[f]=\int_{\Omega} f(\omega) \mathrm{d} \mathbb{Q}(\omega)
$$

whenever the right hand side is well defined.

\subsection{Level II: Rényi’s relative entropy and thermodynamic formalism on $[0,1]$}

For $T \geq 1$ and $\alpha \in \mathbb{R}$, we adopt the shorthand

$$
e_{T}(\alpha):=S_{\alpha}\left(\mathbb{P}_{T} \mid \widehat{\mathbb{P}}_{T}\right),
$$


On entropy production of repeated quantum measurements I

and note that, up to a sign change of its argument,

$$
e_{T}(\alpha)=\log \mathbb{E}\left[\mathrm{e}^{-\alpha \sigma_{T}}\right]
$$

is the cumulant generating function of $\sigma_{T}$. In order to obtain interesting statistical information about the asymptotic behavior of the random variable $\sigma_{T}$, we shall investigate the existence and smoothness properties of the large- $T$ limit of the function $T^{-1} e_{T}(\alpha)$. To get a rough picture of the limiting function, avoiding the more subtle question of its existence, we first describe the basic properties of the function

$$
\mathbb{R} \ni \alpha \mapsto \bar{e}(\alpha)=\limsup _{T \rightarrow \infty} \frac{1}{T} e_{T}(\alpha) \in[-\infty,+\infty] .
$$

According to the general properties of Rényi's relative entropy listed in Section 2.1, the function $\mathbb{R} \ni \alpha \mapsto e_{T}(\alpha)$ is real analytic, convex, vanishing for $\alpha \in\{0,1\}$, non-positive on the interval $[0,1]$ and non-negative on its complement. The symmetry property (2.8) and the invariance property (2.11) further yield

$$
e_{T}(\alpha)=e_{T}(1-\alpha) \text {. }
$$

Finally, from Relations (2.9) and (2.14) we deduce $e_{T}^{\prime}(1)=-e_{T}^{\prime}(0)=\mathbb{E}\left[\sigma_{T}\right]$, which implies the lower bound

$$
e_{T}(\alpha) \geq\left(\left|\alpha-\frac{1}{2}\right|-\frac{1}{2}\right) \mathbb{E}\left[\sigma_{T}\right] .
$$

From the general properties of convex functions (we refer the reader to [Ro] for details) we infer that $\bar{e}$ is a convex function vanishing for $\alpha \in\{0,1\}$. It is non-positive on the interval $[0,1]$ and non-negative on its complement. It satisfies the symmetry

$$
\bar{e}(1-\alpha)=\bar{e}(\alpha)
$$

and the lower bound

$$
\bar{e}(\alpha) \geq\left(\left|\alpha-\frac{1}{2}\right|-\frac{1}{2}\right) \operatorname{ep}(\mathcal{J}, \rho) \geq-\frac{1}{2} \operatorname{ep}(\mathcal{J}, \rho) .
$$

The following dichotomy holds: either $\bar{e}$ is a proper convex function, i.e., $\bar{e}(\alpha)>-\infty$ for all $\alpha$, or it is improper and takes the value $-\infty$ for some $\alpha \in] 0,1[$. In the first case, which is ensured by the condition $\operatorname{ep}(\mathcal{J}, \rho)<\infty$, there exists $\frac{1}{2} \leq \kappa \leq \infty$ such that $\bar{e}$ is continuous on $\left.\mathcal{I}=\right] \frac{1}{2}-\kappa, \frac{1}{2}+\kappa[$ and takes the value $+\infty$ on the (possibly empty) complement of the closure of $\mathcal{I}$. In the second case, $\operatorname{ep}(\mathcal{J}, \rho)=\infty, \bar{e}(\alpha)=-\infty$ for all $\alpha \in] 0,1[$ and $\bar{e}(\alpha)=+\infty$ for all $\alpha \in \mathbb{R} \backslash[0,1]$; see [Ro, Theorem 7.2].

The first results in this section concerns the existence and the characterization of the large- $T$ limit of $T^{-1} e_{T}(\alpha)$ for $\alpha \in[0,1]$. To motivate our approach, note that the variational principle (2.12) implies that for all $T \geq 1$ and $\alpha \in \mathbb{R}$,

$$
\begin{aligned}
\frac{1}{T} e_{T}(\alpha) & =\frac{1}{T} \max _{\mathbb{Q} \in \mathcal{P}}\left(-\alpha \mathbb{Q}\left[\sigma_{T}\right]-S\left(\mathbb{Q}_{T} \mid \mathbb{P}_{T}\right)\right) \\
& =\frac{1}{T} \max _{\mathbb{Q} \in \mathcal{P}}\left(-\alpha \mathbb{Q}\left[\log \mathbb{P}_{T}\right]+\alpha \mathbb{Q}\left[\log \widehat{\mathbb{P}}_{T}\right]-\mathbb{Q}\left[\log \mathbb{Q}_{T}\right]+\mathbb{Q}\left[\log \mathbb{P}_{T}\right]\right) \\
& \geq \max _{\mathbb{Q} \in \mathcal{P}_{\phi}}\left(\frac{1}{T} \mathbb{Q}^{(\alpha)}\left[\log \mathbb{P}_{T}\right]-\frac{1}{T} \mathbb{Q}\left[\log \mathbb{Q}_{T}\right]\right),
\end{aligned}
$$

which indicates that the large- $T$ limit of the functional appearing on the right hand side of this expression may be connected to the limiting cumulant generating function of $\sigma_{T}$. 
Theorem 2.4 (1) For all $\alpha \in[0,1]$ the (possibly infinite) limit

$$
e(\alpha):=\lim _{T \rightarrow \infty} \frac{1}{T} e_{T}(\alpha)
$$

exists, is non-positive, and satisfies $e(0)=e(1)=0$. The function $[0,1] \ni \alpha \mapsto e(\alpha)$ is convex and satisfies the symmetry

$$
e(\alpha)=e(1-\alpha)
$$

We shall call $e(\alpha)$ the entropic pressure of the repeated measurement process $(\mathcal{J}, \rho)$.

(2) The following alternative holds: either $e(\alpha)=-\infty$ for all $\alpha \in] 0,1[$, or $e(\alpha)>-\infty$ for all $\alpha \in[0,1]$.

(3) For any $\mathbb{Q} \in \mathcal{P}_{\phi}$ the (possibly infinite) limit

$$
\varsigma(\mathbb{Q}):=\lim _{T \rightarrow \infty} \mathbb{Q}\left[-\frac{1}{T} \log \mathbb{P}_{T}\right]
$$

exists and is non-negative. The map $\mathcal{P}_{\phi} \ni \mathbb{Q} \mapsto \varsigma(\mathbb{Q})$ is affine, lower semicontinuous, and satisfies $\varsigma(\mathbb{P})=h_{\phi}(\mathbb{P})$.

(4) $\operatorname{For} \mathbb{Q} \in \mathcal{P}_{\phi}$ we set

$$
f(\mathbb{Q}):=h_{\phi}(\mathbb{Q})-\varsigma(\mathbb{Q}) .
$$

The map $\mathcal{P}_{\phi} \ni \mathbb{Q} \mapsto f(\mathbb{Q})$ is affine and upper semicontinuous. It satisfies $f(\mathbb{P})=0$ and

$$
f\left(\mathbb{Q}^{(\alpha)}\right) \leq \bar{e}(\alpha)
$$

for all $\mathbb{Q} \in \mathcal{P}_{\phi}$ and $\alpha \in \mathbb{R}$.

In the remaining statements we assume that $\inf _{\alpha \in[0,1]} e(\alpha)>-\infty$.

(5) For $\alpha \in[0,1]$ one has

$$
e(\alpha)=\sup _{\mathbb{Q} \in \mathcal{P}_{\phi}} f\left(\mathbb{Q}^{(\alpha)}\right)
$$

and the set

$$
\mathcal{P}_{\text {eq }}(\alpha):=\left\{\mathbb{Q} \in \mathcal{P}_{\phi} \mid e(\alpha)=f\left(\mathbb{Q}^{(\alpha)}\right)\right\}
$$

is a non-empty, convex, compact subset of $\mathcal{P}_{\phi}$. It is a Choquet simplex and a face of $\mathcal{P}_{\phi}$. The extreme points of $\mathcal{P}_{\text {eq }}(\alpha)$ are $\phi$-ergodic.

(6) The function $\alpha \mapsto e(\alpha)$ admits a left/right derivative $\partial^{\mp} e(\alpha)$ at each $\left.\alpha \in\right] 0,1[$, and

$$
\partial^{-} e(\alpha)=\inf _{\mathbb{Q} \in \mathcal{P}_{\mathrm{eq}}(\alpha)}(\varsigma(\mathbb{Q})-\varsigma(\widehat{\mathbb{Q}})) \leq \sup _{\mathbb{Q} \in \mathcal{P}_{\mathrm{eq}}(\alpha)}(\varsigma(\mathbb{Q})-\varsigma(\widehat{\mathbb{Q}}))=\partial^{+} e(\alpha)
$$

(7) The left/right derivative of $e(\alpha)$ also exists at $\alpha=0 / 1$ and

$$
\begin{aligned}
& \partial^{+} e(0)=\sup _{\mathbb{Q} \in \mathcal{P}_{\mathrm{eq}}(0)}(\varsigma(\mathbb{Q})-\varsigma(\widehat{\mathbb{Q}})) \geq-\operatorname{ep}(\mathcal{J}, \rho), \\
& \partial^{-} e(1)=\inf _{\mathbb{Q} \in \mathcal{P}_{\mathrm{eq}}(1)}(\varsigma(\mathbb{Q})-\varsigma(\widehat{\mathbb{Q}})) \leq \operatorname{ep}(\mathcal{J}, \rho) .
\end{aligned}
$$


(8) If $\mathbb{P}$ is ergodic, then $\mathcal{P}_{\text {eq }}(0)=\{\mathbb{P}\}, \mathcal{P}_{\text {eq }}(1)=\{\widehat{\mathbb{P}}\}$, and $\partial^{-} e(1)=-\partial^{+} e(0)=\operatorname{ep}(\mathcal{J}, \rho)$.

Remark 1. As already mentioned, the condition $\operatorname{ep}(\mathcal{J}, \rho)<\infty$ ensures that $e(\alpha)>-\infty$ for $\alpha \in$ $[0,1]$. Thus, Remark 3 in Section 2.2 provides a sufficient condition for the validity of Parts (5)-(8). More precisely, if $\Phi_{a}[\mathbb{1}] \geq \epsilon \mathbb{1}$ for some $\epsilon>0$ and all $a \in \mathcal{A}$, then $e(\alpha) \geq \log \ell+\log \epsilon$ for all $\alpha \in[0,1]$. The normalization $\sum_{a \in \mathcal{A}} \Phi_{a}(\mathbb{1})=\mathbb{1}$ ensures that $\epsilon \leq \frac{1}{\ell}$.

Remark 2. The symmetry (2.26) implies that the involution $\Theta$ maps $\mathcal{P}_{\text {eq }}(\alpha)$ onto $\mathcal{P}_{\text {eq }}(1-\alpha)$.

Remark 3. Regarding (2.27), note that for any $\mathbb{Q} \in \mathcal{P}_{\phi}$ such that $\varsigma(\mathbb{Q})$ and $\varsigma(\widehat{\mathbb{Q}})$ are finite,

$$
\varsigma(\mathbb{Q})-\varsigma(\widehat{\mathbb{Q}})=-\lim _{T \rightarrow \infty} \frac{1}{T} \mathbb{Q}\left(\sigma_{T}\right) .
$$

Remark 4. Indicating by a subscript the dependence of the entropic pressure, Remark 4 after Theorem 2.1 extends as follows. The entropic pressure of the product of two instruments is easily seen to be

$$
e_{\left(\mathcal{J}_{1} \otimes \mathcal{J}_{2}, \rho_{1} \otimes \rho_{2}\right)}(\alpha)=e_{\left(\mathcal{J}_{1}, \rho_{1}\right)}(\alpha)+e_{\left(\mathcal{J}_{2}, \rho_{2}\right)}(\alpha),
$$

while the joint concavity of Rényi entropy and Eq. (2.21) yield the following inequality for general sums:

$$
e_{\left(\mathcal{J}_{1} \oplus \mathcal{J}_{2}, \mu \rho_{1} \oplus(1-\mu) \rho_{2}\right)}(\alpha) \geq \mu e_{\left(\mathcal{J}_{1}, \rho_{1}\right)}(\alpha)+(1-\mu) e_{\left(\mathcal{J}_{2}, \rho_{2}\right)}(\alpha) .
$$

In the special case of a disjoint sum the identity

$$
e_{\left(\mathcal{J}_{1} \oplus \mathcal{J}_{2}, \mu \rho_{1} \oplus(1-\mu) \rho_{2}\right)}(\alpha)=\max \left(e_{\left(\mathcal{J}_{1}, \rho_{1}\right)}(\alpha), e_{\left(\mathcal{J}_{2}, \rho_{2}\right)}(\alpha)\right)
$$

holds. If $\mathcal{J}_{2} \succ \mathcal{J}_{1}$, then Inequality (2.10) shows that

$$
e_{\left(\mathcal{J}_{1}, \rho\right)}(\alpha) \geq e_{\left(\mathcal{J}_{2}, \rho\right)}(\alpha) .
$$

It follows that for compositions we have

$$
e_{\left(\mathcal{J}_{1} \circ \mathcal{J}_{2}, \rho\right)}(\alpha) \geq e_{\left(\mathcal{J}_{1}, \rho\right)}(\alpha) .
$$

Finally, if $\lim \left(\mathcal{J}_{n}, \rho_{n}\right)=(\mathcal{J}, \rho)$, then

$$
\limsup _{n \rightarrow \infty} e_{\left(\mathcal{J}_{n}, \rho_{n}\right)}(\alpha) \leq e_{(\mathcal{J}, \rho)}(\alpha) .
$$

To prove this inequality, one uses that

$$
e_{\left(\mathcal{J}_{n}, \rho_{n}\right)}(\alpha)=\inf _{T \geq 1} \frac{e_{T,\left(\mathcal{J}_{n}, \rho_{n}\right)}(\alpha)-\log \lambda_{n}}{T}
$$

(see the proof of Part (1) of Theorem 2.4), and argues in the same way as in the proof of the respective part of Remark 4 after Theorem 2.1.

To achieve a better control of the fluctuations of the entropic functional $\sigma_{T}$ and to derive Chernoff and Hoeffding error exponents for the hypothesis testing of the arrow of time, we must improve Theorem 2.4 in two ways: (a) by obtaining more information on the smoothness of the entropic pressure $e(\alpha)$, which, in the language of thermodynamics, amounts to investigating the (non-)existence of dynamical phase transitions; and (b) by extending our control of the limit (2.25) outside of the interval $[0,1]$. In the next two sections we settle these goals. 


\subsection{Differentiability on $] 0,1[$}

Theorems 2.1, 2.3, 2.4 and Proposition 2.2 are very general results. They hold for any $\mathbb{P} \in \mathcal{P}_{\phi}$ as long as supp $\mathbb{P}_{T}=\operatorname{supp} \widehat{\mathbb{P}}_{T}$ for all $T \geq 1$ and the following structural inequality holds ${ }^{10}$ : for some $C>0$ and all $T, T^{\prime} \geq 1$,

$$
\mathbb{P}_{T+T^{\prime}} \leq C \mathbb{P}_{T} \mathbb{P}_{T^{\prime}} \circ \phi^{T}
$$

Note that if (2.30) holds for $\mathbb{P}$, then it automatically holds for $\widehat{\mathbb{P}}$ with the same constant $C$. Although property (2.30) could be difficult to establish for generic dynamical systems, it always holds for the systems associated with repeated measurement process satisfying the regularity assumptions (A) and (B); see Lemma 3.4. This is the reason that, until this point, we did not need any additional assumptions on our model.

To proceed with our analysis and establish smoothness of the entropic pressure on the interval $] 0,1[$, we need to complement the inequality (2.30) with a suitable lower bound.

To put our assumptions in perspective, we start by recalling the notion of a Gibbs measure as introduced by Bowen [Bo1, Bo2]. A measure $\mathbb{P} \in \mathcal{P}_{\phi}$ is called Gibbs if there exists a Hölder continuous function $\varphi: \Omega \rightarrow \mathbb{R}$ and a constant $C>0$ such that for all $T \geq 1$,

$$
C^{-1} \mathrm{e}^{-\sum_{t=0}^{T-1} \varphi \circ \phi^{t}} \leq \mathbb{P}_{T} \leq C \mathrm{e}^{-\sum_{t=0}^{T-1} \varphi \circ \phi^{t}} .
$$

The thermodynamic formalism of Gibbs measures is well-understood and is easily adapted to the study of entropy production. Indeed, the first proof of the fluctuation relation/theorem was done in this setting [GC1, GC2]; for an exposition of the full theory and references we refer the reader to [JPR, MV]. One easily shows that a Gibbs measure satisfies the lower and upper quasi-Bernoulli properties

$$
C^{-3} \mathbb{P}_{T} \mathbb{P}_{T^{\prime}} \circ \phi^{T} \leq \mathbb{P}_{T+T^{\prime}} \leq C^{3} \mathbb{P}_{T} \mathbb{P}_{T^{\prime}} \circ \phi^{T} .
$$

However, except in special cases, the measures $\mathbb{P}$ arising in repeated measurement processes do not satisfy the above quasi-Bernoulli lower bound and hence are not Gibbs. Although the study of the thermodynamical formalism for non-Gibbsian measures can be traced back to the celebrated program of Dobrushin [Do, DoS] (see the reviews [Fe, LN, VE] for additional information), the approach we adopt in this work was developed only relatively recently, and is called the subadditive thermodynamic formalism; see [BaL, BV, CFH, CZC, Fa, FS, Fe1, Fe2, Fe3, FL, FK, IY, KW]. In this approach, one assumes the upper bound (2.30) and, depending on a setting, an appropriate lower bound, while completely abandoning the Gibbs condition (2.31). We shall proceed similarly, keeping in mind that in our case the upper bound is always satisfied, while an effective lower bound has to be based on an assumption that is suited for study of entropy production and is natural in the context of repeated quantum measurement processes.

To formulate this assumption we introduce some additional notation. We denote by

$$
\Omega_{\mathrm{fin}}=\bigcup_{T \geq 0} \Omega_{T}
$$

the set of finite words. For $\boldsymbol{\omega} \in \Omega_{\text {fin }}$ we set $|\boldsymbol{\omega}|=T$ and $\mathbb{P}(\boldsymbol{\omega})=\mathbb{P}_{T}\left(\omega_{1}, \ldots, \omega_{T}\right)$ whenever $\boldsymbol{\omega}=\left(\omega_{1}, \ldots, \omega_{T}\right){ }^{11}$

\footnotetext{
${ }^{10}$ In the literature, the inequality (2.30) is sometimes called the upper quasi-Bernoulli property.

${ }^{11}$ By convention $\boldsymbol{\omega} \in \Omega_{0}$ is the empty word, i.e., $|\boldsymbol{\omega}|=0$ and $\mathbb{P}(\boldsymbol{\omega})=1$.
} 
Assumption (C) There exists $\tau \geq 0$ such that

$$
C_{\tau}=\inf _{(\boldsymbol{\omega}, \boldsymbol{\nu}) \in \Omega_{\mathrm{fin}} \times \Omega_{\mathrm{fin}}} \max _{\substack{\boldsymbol{\xi} \in \Omega_{\mathrm{fin}} \\|\boldsymbol{\xi}| \leq \tau}} \frac{\mathbb{P}(\boldsymbol{\omega} \boldsymbol{\xi} \boldsymbol{\nu}) \widehat{\mathbb{P}}(\boldsymbol{\omega} \boldsymbol{\xi} \boldsymbol{\nu})}{\mathbb{P}(\boldsymbol{\nu}) \widehat{\mathbb{P}}(\boldsymbol{\omega}) \widehat{\mathbb{P}}(\boldsymbol{\nu})}>0 .
$$

One of the main results of this work is:

Theorem 2.5 Suppose that Assumption (C) holds. Then, for all $\alpha \in] 0,1\left[\right.$, the set $\mathcal{P}_{\mathrm{eq}}(\alpha)$ is a singleton. In particular, the function $] 0,1[\ni \alpha \mapsto e(\alpha)$ is differentiable.

Although Assumption (C) may look technical, it is a natural optimal condition under which the subadditive thermodynamic formalism gives that $\mathcal{P}_{\text {eq }}(\alpha)$ is a singleton for $\left.\alpha \in\right] 0,1[$. Moreover, Theorem 2.5 and its proof extend to any $\mathbb{P} \in \mathcal{P}_{\phi}$ for which Assumptions (B) and (C) hold and which satisfies the bound (2.30).

As we shall discuss in [BJPP1], Assumption (C) is typically easy to verify in applications to concrete examples. The next two propositions give sufficient conditions for (C) that can be expressed directly in terms of the instrument $\left\{\Phi_{a}\right\}_{a \in \mathcal{A}}$.

Proposition 2.6 Suppose that there exists an OR process $(\widehat{\mathcal{J}}, \widehat{\rho})$ with instrument $\widehat{\mathcal{J}}=\left\{\widehat{\Phi}_{a}\right\}_{a \in \mathcal{A}}$ such that the completely positive map $\Psi: \mathcal{B}(\mathcal{H} \otimes \mathcal{H}) \rightarrow \mathcal{B}(\mathcal{H} \otimes \mathcal{H})$ defined by

$$
\Psi=\sum_{a \in \mathcal{A}} \Phi_{a} \otimes \widehat{\Phi}_{a}
$$

is irreducible. ${ }^{12}$ Then Assumption (C) holds.

Remark 1. One easily shows that $\Psi \leq \Phi \otimes \widehat{\Phi}$, from which one deduces that Assumption (C) implies the irreducibility of $\Phi$. By Theorem 1.1, the latter condition, in turn, implies that $\mathbb{P}$ is $\phi$-ergodic.

Remark 2. If $(\widehat{\mathcal{J}}, \widehat{\rho})$ is the canonical OR process (1.3) and one of the $\Phi_{a}$ 's is irreducible, then Proposition 2.6 applies and Assumption (C) holds. Thus, given any process $(\mathcal{J}, \rho), \mathcal{J}=\left\{\Phi_{a}\right\}_{a \in \mathcal{A}}$, and a completely positive unital irreducible map $\Xi$ satisfying $\Xi^{*}(\rho)=\rho$, the instrument $\mathcal{J}_{\epsilon}=\{(1-$ $\left.\epsilon) \Phi_{a}, \epsilon \Xi\right\}_{a \in \mathcal{A}}$, where $\left.\epsilon \in\right] 0,1[$, together with its canonical OR instrument, satisfies Assumption (C). The parameter $\epsilon$ can be interpreted as the probability that at each time $t=1,2, \cdots$, no measurement is made, or that the measurement result is lost/not read. If $e_{\epsilon}(\alpha)$ is the entropic pressure of $\left(\mathcal{J}_{\epsilon}, \rho\right)$ and $e(\alpha)$ of $(\mathcal{J}, \rho)$, then for all $\alpha \in[0,1]$,

$$
e_{\epsilon}(\alpha) \geq \log (1-\epsilon)+e(\alpha)
$$

while (2.29) gives $\lim _{\epsilon \downarrow 0} e_{\epsilon}(\alpha) \leq e(\alpha)$. Hence,

$$
\lim _{\epsilon \downarrow 0} e_{\epsilon}(\alpha)=e(\alpha) .
$$

If $\inf _{\alpha \in[0,1]} e(\alpha)>-\infty$, then the convexity gives that the convergence (2.33) is uniform on $[0,1]$, and that for $\alpha \in] 0,1[$,

$$
\partial^{-} e(\alpha) \leq \liminf _{\epsilon \downarrow 0} e_{\epsilon}^{\prime}(\alpha) \leq \limsup _{\epsilon \downarrow 0} e_{\epsilon}^{\prime}(\alpha) \leq \partial^{+} e(\alpha),
$$

\footnotetext{
${ }^{12}$ Recall that $\Psi$ is irreducible if $\Psi[P] \leq \lambda P$ for some orthogonal projection $P \in \mathcal{B}(\mathcal{H} \otimes \mathcal{H})$ and some $\lambda>0$ implies $P \in\{0, \mathbb{1}\}$.
} 
while for $\alpha=0 / 1$,

$$
\partial^{+} e(0) \geq \limsup _{\epsilon \downarrow 0} e_{\epsilon}^{\prime}(0), \quad \partial^{-} e(1) \leq \liminf _{\epsilon \downarrow 0} e_{\epsilon}^{\prime}(0) .
$$

Using the canonical OR process (1.3), and invoking [JPW, Theorem 2.1], we obtain the following simple algebraic criterion for validity of Proposition 2.6 and Assumption (C).

Proposition 2.7 Let

$$
\Phi_{a}[X]=\sum_{k=1}^{K_{a}} V_{a, k}^{*} X V_{a, k}
$$

be a Kraus decomposition of the instrument $\mathcal{J}=\left\{\Phi_{a}\right\}_{a \in \mathcal{A}}$ and define

$$
W_{a, j, k}=V_{a, j} \otimes V_{\theta(a), k}^{*} .
$$

If the family $\left\{W_{a, j, k} \mid a \in \mathcal{A}, j \in \llbracket 1, K_{a} \rrbracket, k \in \llbracket 1, K_{\theta(a)} \rrbracket\right\}$ acts irreducibly on $\mathcal{H} \otimes \mathcal{H}$, i.e., if the only subspaces of $\mathcal{H} \otimes \mathcal{H}$ which are invariant under all $W_{a, j, k}$ are $\{0\}$ and $\mathcal{H} \otimes \mathcal{H}$ itself, then the map (2.32) is irreducible and Assumption (C) holds.

\subsection{Full thermodynamic formalism}

To the best of our knowledge, Assumption (C) is not sufficient to extend the thermodynamic formalism of Theorems 2.4 and 2.5 to all $\alpha \in \mathbb{R}$. To deal with this point we strengthen $(\mathrm{C})$ as follows:

\section{Assumption (D)}

$$
D_{0}=\inf _{(\boldsymbol{\omega}, \boldsymbol{\nu}) \in \Omega_{\mathrm{fin}} \times \Omega_{\mathrm{fin}}} \frac{\mathbb{P}(\boldsymbol{\omega} \boldsymbol{\nu})}{\mathbb{P}(\boldsymbol{\omega}) \mathbb{P}(\boldsymbol{\nu})}>0
$$

Note that if (D) holds for $\mathbb{P}$, then it also holds for $\widehat{\mathbb{P}}$ with the same constant $D_{0}$.

Theorem 2.8 Suppose that Assumption (D) holds. Then:

(1) The limit

$$
e(\alpha):=\lim _{T \rightarrow \infty} \frac{1}{T} e_{T}(\alpha)
$$

exists for all $\alpha \in \mathbb{R}$, and the function $\mathbb{R} \ni \alpha \mapsto e(\alpha)$ is differentiable.

(2) For any $\alpha \in \mathbb{R}$, there exists a unique $\mathbb{Q}_{\alpha} \in \mathcal{P}_{\phi}$ such that

$$
e(\alpha)=f\left((1-\alpha) \mathbb{Q}_{\alpha}+\alpha \widehat{\mathbb{Q}}_{\alpha}\right)=\sup _{\mathbb{Q} \in \mathcal{P}_{\phi}} f((1-\alpha) \mathbb{Q}+\alpha \widehat{\mathbb{Q}})
$$

Moreover, $\mathbb{Q}_{\alpha}$ is $\phi$-ergodic and

$$
e^{\prime}(\alpha)=\varsigma\left(\mathbb{Q}_{\alpha}\right)-\varsigma\left(\widehat{\mathbb{Q}}_{\alpha}\right)
$$


On entropy production of repeated quantum measurements I

In our setting Assumption (D) plays the role of the uniform hyperbolicity assumption in dynamical system theories and replaces/generalizes the Gibbs condition (2.31). As we shall see in Section 2.8.2, Theorem 2.4 yields a global Fluctuation Theorem for repeated quantum measurement process. All known examples for which a global Fluctuation Theorem is proven are uniformly hyperbolic in a suitable sense. We believe that (D) is the optimal general assumption for validity of Theorem 2.4 and the global Fluctuation Theorem.

Theorem 2.5 and its proof extend to any $\mathbb{P} \in \mathcal{P}_{\phi}$ for which Assumptions (B), (D) hold and which satisfies the bound (2.30). The next proposition gives sufficient condition for (D) in terms of the instrument $\left\{\Phi_{a}\right\}_{a \in \mathcal{A}}$.

Proposition 2.9 Suppose that the map $\Phi_{a}$ is positivity improving ${ }^{13}$ for all $a \in \mathcal{A}$. Then (D) holds.

Remark 1. Given any process $(\mathcal{J}, \rho), \mathcal{J}=\left\{\Phi_{a}\right\}_{a \in \mathcal{A}}$, and unital completely positive positivity improving maps $\left\{\Psi_{a}\right\}_{a \in \mathcal{A}}$ satisfying $\Psi_{a}^{*}(\rho)=\rho$, the instrument

$$
\mathcal{J}_{\epsilon}=\left\{(1-\epsilon) \Phi_{a}+\frac{\epsilon}{\ell} \Psi_{a}\right\}_{a \in \mathcal{A}},
$$

where $\epsilon \in] 0,1\left[\right.$, satisfies the assumption of the proposition. The deformation $\mathcal{J}_{\epsilon}$ of the original instrument $\mathcal{J}$ can be interpreted as an effect of a "noise" inherent in the measurement process. One easily verifies that for all $\alpha \in \mathbb{R}$, $\liminf _{\epsilon \downarrow 0} e_{\epsilon}(\alpha) \geq \bar{e}(\alpha)$, while (2.33)-(2.35) remain valid for $\alpha \in[0,1]$.

Remark 2. It is likely that under the assumption of the last proposition the function $\alpha \mapsto e(\alpha)$ is real analytic. The proof of such a result would require an adaptation of the transfer operator techniques to our settting [Ba]. This point will be further discussed in [BJPP3].

\subsection{Level II: Large Deviations}

In this section we use Theorems $2.4,2.5$ and 2.8 to study fluctuations of the entropy production functional $\sigma_{T}$.

\subsubsection{Basic large deviations estimates}

Assuming only Conditions (A)-(B) and $\inf _{\alpha \in[0,1]} e(\alpha)>-\infty$, the following variant of the Large Deviation Principle follows from Theorem $2.4^{14}$.

Recall that $\mathcal{I}=] \frac{1}{2}-\kappa, \frac{1}{2}+\kappa[$ is the interior of the essential domain $\{\alpha \in \mathbb{R} \mid \bar{e}(\alpha)<\infty\}$. Set

$$
s_{ \pm}=-\partial^{ \pm} \bar{e}\left(\frac{1}{2} \mp \kappa\right),
$$

and note that

$$
-\infty \leq s_{-} \leq-\operatorname{ep}(\mathcal{J}, \rho) \leq \operatorname{ep}(\mathcal{J}, \rho) \leq s_{+} \leq+\infty
$$

For $s \in \mathbb{R}$, set

$$
I(s)=\sup _{\alpha \in \mathbb{R}}(\alpha s-\bar{e}(-\alpha))=-\inf _{\alpha \in \mathbb{R}}(\alpha s+\bar{e}(\alpha)) .
$$

\footnotetext{
${ }^{13}$ A positive map $\Psi: \mathcal{B}(\mathcal{H}) \rightarrow \mathcal{B}(\mathcal{H})$ is positivity improving if $\Psi[X]>0$ for all $X \geq 0$.

${ }^{14}$ Note that $\bar{e}(\alpha)=e(\alpha)$ for $\alpha \in[0,1]$.
} 
The function $\mathbb{R} \ni s \mapsto I(s)$ is convex, finite and non-negative. It vanishes at $s=\operatorname{ep}(\mathcal{J}, \rho)$ and is non-increasing (resp. non-decreasing) for $s<\operatorname{ep}(\mathcal{J}, \rho)$ (resp. $s>\operatorname{ep}(\mathcal{J}, \rho)$ ). It satisfies

$$
I(-s)-I(s)=s
$$

as a consequence of the symmetry (2.23). By well-known properties of the Fenchel-Legendre transform,

$$
-I(s)= \begin{cases}\bar{e}\left(\frac{1}{2}+\kappa\right)+\left(\frac{1}{2}+\kappa\right) s & \text { if } s \leq s_{-} \\ \bar{e}(\alpha)+\alpha s & \text { if } s \in-\partial \bar{e}(\alpha) \text { for some } \alpha \in \mathcal{I} \\ \bar{e}\left(\frac{1}{2}-\kappa\right)+\left(\frac{1}{2}-\kappa\right) s & \text { if } s \geq s_{+},\end{cases}
$$

where $\partial \bar{e}(\alpha)=\left[\partial^{-} \bar{e}(\alpha), \partial^{+} \bar{e}(\alpha)\right]$ denotes the subdifferential of $\bar{e}$ at $\alpha$.

Theorem 2.10 Suppose that $\inf _{\alpha \in[0,1]} e(\alpha)>-\infty$. Then:

(1) For any closed subset $C \subset \mathbb{R}$ such that $\sup C<\infty$,

$$
\limsup _{T \rightarrow \infty} \frac{1}{T} \log \mathbb{P}_{T}\left(\left\{\omega \in \Omega_{T} \mid \frac{1}{T} \sigma_{T}(\omega) \in C\right\}\right) \leq-\inf _{s \in C} I(s) .
$$

Moreover, the same estimate holds for all closed sets $C \subset \mathbb{R}$ provided $\kappa>\frac{1}{2}$.

(2) If $s \in-\partial e(\alpha)$ for some $\alpha \in] 0,1[$, then

$$
\liminf _{T \rightarrow \infty} \frac{1}{T} \log \mathbb{P}_{T}\left(\left\{\omega \in \Omega_{T} \mid \frac{1}{T} \sigma_{T}(\omega)<s\right\}\right) \geq-I\left(-\partial^{+} e(\alpha)\right) .
$$

Remark. This is a standard large deviation result $[\mathrm{dH}, \mathrm{DZ}, \mathrm{El}]^{15}$, and we have stated it for reason of completeness. The same remark applies to Theorem 2.11 below.

\subsubsection{A local and a global Fluctuation Theorem}

Theorems 2.5 and 2.8 allow us to refine the large deviations estimates of the previous section and to obtain a full large deviations principle.

Theorem 2.11 (1) If Assumption (C) holds, then for any open set $O \subset]-\operatorname{ep}(\mathcal{J}, \rho), \operatorname{ep}(\mathcal{J}, \rho)[$,

$$
\lim _{T \rightarrow \infty} \frac{1}{T} \log \mathbb{P}_{T}\left(\left\{\omega \in \Omega_{T} \mid \frac{1}{T} \sigma_{T}(\omega) \in O\right\}\right)=-\inf _{s \in O} I(s) .
$$

(2) Under Assumption (D), Relation (2.38) holds for any open set $O \subset \mathbb{R}$.

Parts (1) and (2) of Theorem 2.11 together with the relation (2.36) constitute, respectively, the local and global Fluctuation Theorem for our model; see [ECM, ES, GC1, GC2] for foundational works on the subject and [JPR, Ku1, LS, Ma1, Ma2, MN, MV, RM] for additional information.

\footnotetext{
${ }^{15}$ See also [JOPP, JOPS] for a pedagogical exposition.
} 
On entropy production of repeated quantum measurements I

Remark. The following elementary observations provide a background for the Fluctuation Theorem. Denote by $Q_{T}$ the law of the random variable $\frac{1}{T} \sigma_{T}$ w.r.t. $\mathbb{P}_{T}$ :

$$
Q_{T}(s)=\mathbb{P}_{T}\left(\left\{\omega \in \Omega_{T} \mid \sigma_{T}(\omega)=s T\right\}\right) .
$$

Obviously, $Q_{T}(s) \neq 0 \Leftrightarrow Q_{T}(-s) \neq 0$ and

$$
\int s \mathrm{~d} Q_{T}(s)=\frac{1}{T} \mathbb{E}\left[\sigma_{T}\right] \geq 0 .
$$

The relation (2.22) can be written as

$$
\int \mathrm{e}^{-\alpha \sigma_{T}} \mathrm{~d} \mathbb{P}_{T}=\int \mathrm{e}^{-(1-\alpha) \sigma_{T}} \mathrm{~d} \mathbb{P}_{T}
$$

and so for any $s$,

$$
Q_{T}(-s)=\mathrm{e}^{-T s} Q_{T}(s) .
$$

The relation (2.39) is the Jarzynski inequality in our setting. The normalization

$$
1=\int \mathrm{d} \widehat{\mathbb{P}}_{T}=\int \mathrm{e}^{-\sigma_{T}} \mathrm{dP}_{T}=\int \mathrm{e}^{-s T} \mathrm{~d} Q_{T}(s)
$$

is the Jarzynski identity, and (2.40) is the finite time Fluctuation Relation. Needless to say, the above elementary relations are completely general and hold for any $\mathbb{Q} \in \mathcal{P}_{\phi}$ and the associated entropy production observable. As emphasized in [GC1, GC2], the mathematically and physically non-trivial aspects of the Fluctuation Theorem emerge through the Large Deviation Principle and the induced symmetry (2.36) of the rate function; see [JNPPS, JPS] for references and additional information regarding this point.

\subsection{Level II: Hypothesis testing}

By Remark 2 after Theorem 2.1, if $\mathbb{P}$ is $\phi$-ergodic and $\operatorname{ep}(\mathcal{J}, \rho)>0$, then $\mathbb{P}$ and $\widehat{\mathbb{P}}$ are mutually singular, i.e., concentrated on disjoint subsets of $\Omega$, whereas Assumption (B) ensures that their marginals $\mathbb{P}_{T}$ and $\widehat{\mathbb{P}}_{T}$ share a common support for all $T \geq 1$. Hypothesis testing error exponents quantify the asymptotic separation of $\mathbb{P}_{T}$ and $\widehat{\mathbb{P}}_{T}$ as $T \rightarrow \infty$. In our setting, given the physical interpretation of $\mathbb{P}_{T}$ and $\widehat{\mathbb{P}}_{T}$ in terms of the process $(\mathcal{J}, \rho)$ and its outcome reversal $(\widehat{\mathcal{J}}, \widehat{\rho})$, one can say more colloquially that they substantiate the emergence of the arrow of time in repeated quantum measurement processes.

The Chernoff error exponents of the pair $(\mathbb{P}, \widehat{\mathbb{P}})$ are defined by

$$
\underline{c}=\liminf _{T \rightarrow \infty} \frac{1}{T} \log c_{T}, \quad \bar{c}=\limsup _{T \rightarrow \infty} \frac{1}{T} \log c_{T},
$$

where

$$
c_{T}=\frac{1}{4}\left(2-\sum_{\omega \in \Omega_{T}}\left|\mathbb{P}_{T}(\omega)-\widehat{\mathbb{P}}_{T}(\omega)\right|\right) .
$$

In the context of hypothesis testing, the number $c_{T}$ arises as follows. Let $(\widehat{\mathcal{J}}, \widehat{\rho})$ be an outcome reversal of $(\mathcal{J}, \rho)$. Consider the following two competing hypotheses: 
Hypothesis I : The observed quantum measurements are described by the process $(\mathcal{J}, \rho)$.

Hypothesis II : The observed quantum measurements are described by the OR process $(\widehat{\mathcal{J}}, \widehat{\rho})$.

By observing the first $T$ outcomes of the measurements we wish to determine with minimal error probability which of these two hypotheses is correct. More precisely, a test $\mathcal{T}$ is a subset of $\Omega_{T}$ and its purpose is to discriminate between the two hypotheses. If the outcome $\omega$ of the first $T$ measurements is in $\mathcal{T}$, one accepts I and rejects II. Otherwise, one accepts II and rejects I. To a given test $\mathcal{T}$ one can associate two kinds of errors. A type-I error occurs when I holds but $\omega \notin \mathcal{T}$. The conditional probability of such an error is $\mathbb{P}_{T}\left(\mathcal{T}^{c}\right)$, where $\mathcal{T}^{c}$ is the complement of $\mathcal{T}$ in $\Omega_{T}$. If II holds and $\omega \in \mathcal{T}$, we get a type-II error, with conditional probability $\widehat{\mathbb{P}}_{T}(\mathcal{T})$.

Assuming that the Bayesian probabilities assigned to the Hypothesis I and II are $\frac{1}{2}^{16}$, the total error probability is equal to $\frac{1}{2} \mathbb{P}_{T}\left(\mathcal{T}^{c}\right)+\frac{1}{2} \widehat{\mathbb{P}}_{T}(\mathcal{T})$ which we wish to minimize over $\mathcal{T}$. The Neyman-Pearson lemma identifies the minimizer: if

$$
\underline{\mathcal{T}}_{T}=\left\{\omega \in \Omega_{T} \mid \mathbb{P}_{T}(\omega) \geq \widehat{\mathbb{P}}_{T}(\omega)\right\}
$$

then for any test $\mathcal{T} \subset \Omega_{T}$,

$$
\begin{aligned}
\frac{1}{2} \mathbb{P}_{T}\left(\mathcal{T}^{c}\right)+\frac{1}{2} \widehat{\mathbb{P}}_{T}(\mathcal{T}) & =\frac{1}{2}-\frac{1}{2}\left(\mathbb{P}_{T}(\mathcal{T})-\widehat{\mathbb{P}}_{T}(\mathcal{T})\right) \geq \frac{1}{2}-\frac{1}{2} \sum_{\omega \in \mathcal{T} \cap \mathcal{I}_{T}}\left(\mathbb{P}_{T}(\omega)-\widehat{\mathbb{P}}_{T}(\omega)\right) \\
& =\frac{1}{2}-\frac{1}{2} \sum_{\omega \in \mathcal{T}}\left(\mathbb{P}_{T}(\omega)-\widehat{\mathbb{P}}_{T}(\omega)\right)_{+} \geq \frac{1}{2}-\frac{1}{2} \sum_{\omega \in \Omega_{T}}\left(\mathbb{P}_{T}(\omega)-\widehat{\mathbb{P}}_{T}(\omega)\right)_{+} \\
& =\frac{1}{4}\left(2-\sum_{\omega \in \Omega_{T}}\left|\mathbb{P}_{T}(\omega)-\widehat{\mathbb{P}}_{T}(\omega)\right|\right)=c_{T} .
\end{aligned}
$$

Observing that the two previous inequalities are saturated for $\mathcal{T}=\underline{\mathcal{I}}_{T}$ one concludes that

$$
\frac{1}{2} \mathbb{P}_{T}\left(\underline{\mathcal{T}}_{T}^{c}\right)+\frac{1}{2} \widehat{\mathbb{P}}_{T}\left(\underline{\mathcal{T}}_{T}\right)=c_{T}
$$

Note also that the relation $\underline{\mathcal{T}}_{T}^{c} \subset \Theta_{T}\left(\underline{\mathcal{I}}_{T}\right)$ implies that $\widehat{\mathbb{P}}_{T}\left(\underline{\mathcal{I}}_{T}\right) \geq \mathbb{P}_{T}\left(\underline{\mathcal{T}}_{T}^{c}\right)$.

Theorem 2.12 (1)

$$
\bar{c} \leq e\left(\frac{1}{2}\right),
$$

and in particular $\bar{c}<0$ in cases where $\operatorname{ep}(\mathcal{J}, \rho)>0$.

(2) If $\inf _{\alpha \in[0,1]} e(\alpha)>-\infty$, then

$$
\underline{c} \geq e\left(\frac{1}{2}\right)-\frac{1}{2} \partial^{+} e\left(\frac{1}{2}\right) .
$$

(3) If Assumption (C) holds, then

$$
\underline{c}=\bar{c}=\lim _{T \rightarrow \infty} \frac{1}{T} \log c_{T}=e\left(\frac{1}{2}\right) .
$$

\footnotetext{
${ }^{16}$ The discussion of the Chernoff error exponents easily extends to the case where these probabilities are $p$ and $1-p$ for any $p \in] 0,1[$.
} 
On entropy production of repeated quantum measurements I

Remark. The symmetry (2.26) implies that the convex function $e(\alpha)$ has a global minimum at $\alpha=\frac{1}{2}$. Thus, if $e(\alpha)$ is differentiable at $\alpha=\frac{1}{2}$, then $e^{\prime}\left(\frac{1}{2}\right)=0$. If $e(\alpha)$ is finite on ]0,1[, then it is also differentiable on ]0,1[ outside a countable set, and one may expect that for a "generic" instrument one has $\partial^{+} e\left(\frac{1}{2}\right)=e^{\prime}\left(\frac{1}{2}\right)=0$, in which case the conclusion of Part (3) holds.

Theorem 2.12 provides a quantitative estimate for the emergence of the arrow of time. Eq. (2.42) yields

$$
\limsup _{T \rightarrow \infty} \frac{1}{T} \log \mathbb{P}_{T}\left(\underline{\mathcal{T}}_{T}^{c}\right) \leq \bar{c}, \quad \limsup _{T \rightarrow \infty} \frac{1}{T} \log \widehat{\mathbb{P}}_{T}\left(\underline{\mathcal{T}}_{T}\right)=\bar{c} .
$$

Thus, if $\operatorname{ep}(\mathcal{J}, \rho)>0$, then the marginals $\mathbb{P}_{T}$ and $\widehat{\mathbb{P}}_{T}$ respectively concentrate on the complementary subsets $\underline{\mathcal{T}}_{T}$ and $\underline{\mathcal{T}}_{T}^{c}$, with an exponential rate $\leq \bar{c} \leq e\left(\frac{1}{2}\right)<-\operatorname{ep}(\mathcal{J}, \rho)$. Note also that if Assumption C is satisfied, then

$$
\lim _{T \rightarrow \infty} \frac{1}{T} \log \mathbb{P}_{T}\left(\underline{\mathcal{T}}_{T}^{c}\right)=\lim _{T \rightarrow \infty} \frac{1}{T} \log \widehat{\mathbb{P}}_{T}\left(\underline{\mathcal{T}}_{T}\right)=e\left(\frac{1}{2}\right)
$$

With the above interpretation of $\mathbb{P}_{T}\left(\mathcal{T}_{T}^{c}\right)$ and $\widehat{\mathbb{P}}_{T}\left(\mathcal{T}_{T}\right)$ for $\mathcal{T}_{T} \subset \Omega_{T}$, the number $s_{T}(\epsilon)$ introduced in Section 2.3 is the minimal probability of type-II errors that can be achieved by a test which ensures that type-I errors have a maximal probability $\epsilon \in] 0,1[$. Thus, the Stein error exponents control the exponential decay of type-II errors, $\mathrm{e}^{\underline{s}(\epsilon) T} \lesssim \widehat{\mathbb{P}}_{T}\left(\mathcal{T}_{T}^{c}\right) \lesssim \mathrm{e}^{\bar{s}(\epsilon) T}$ as $T \rightarrow \infty$, in such tests.

The Hoeffding error exponents serve a similar purpose, but with a tighter constraint on the family $\left\{\mathcal{T}_{T}\right\}_{T \geq 1}$ of tests which are required to ensure exponential decay of type-I errors with a minimal rate $s \geq 0$. More precisely,

$$
\begin{aligned}
& \bar{h}(s)=\inf _{\left\{\mathcal{T}_{T}\right\}}\left\{\limsup _{T \rightarrow \infty} \frac{1}{T} \log \widehat{\mathbb{P}}_{T}\left(\mathcal{T}_{T}\right) \mid \limsup _{T \rightarrow \infty} \frac{1}{T} \log \mathbb{P}_{T}\left(\mathcal{T}_{T}^{c}\right)<-s\right\}, \\
& \underline{h}(s)=\inf _{\left\{\mathcal{T}_{T}\right\}}\left\{\liminf _{T \rightarrow \infty} \frac{1}{T} \log \widehat{\mathbb{P}}_{T}\left(\mathcal{T}_{T}\right) \mid \limsup _{T \rightarrow \infty} \frac{1}{T} \log \mathbb{P}_{T}\left(\mathcal{T}_{T}^{c}\right)<-s\right\}, \\
& h(s)=\inf _{\left\{\mathcal{T}_{T}\right\}}\left\{\lim _{T \rightarrow \infty} \frac{1}{T} \log \widehat{\mathbb{P}}_{T}\left(\mathcal{T}_{T}\right) \mid \limsup _{T \rightarrow \infty} \frac{1}{T} \log \mathbb{P}_{T}\left(\mathcal{T}_{T}^{c}\right)<-s\right\},
\end{aligned}
$$

where in the last case the infimum is taken over all families of tests for which $\lim _{T \rightarrow \infty} \frac{1}{T} \log \widehat{\mathbb{P}}_{T}\left(\mathcal{T}_{T}\right)$ exists. The Hoeffding error exponents satisfy $\underline{h}(s) \leq \bar{h}(s) \leq h(s)$ and have the same value if the roles of $\mathbb{P}$ and $\widehat{\mathbb{P}}$ are exchanged. Moreover, the functions $\underline{h}(s), \bar{h}(s), h(s)$ are upper semicontinuous and right-continuous; see [JOPS, Proposition 6.3].

For $s \geq 0$ set

$$
\psi(s)=-\sup _{\alpha \in[0,1[} \frac{-s \alpha-e(\alpha)}{1-\alpha} .
$$

If $e(\alpha)$ is finite on $[0,1]$, then $\psi(0)=-\partial^{-} e(1)=-\operatorname{ep}(\mathcal{J}, \rho)$. Moreover, $\psi$ is concave, increasing, and finite on $] 0, \infty[$.

Theorem 2.13 Suppose that Assumption (C) holds. Then for $s \geq 0$,

$$
\underline{h}(s)=\bar{h}(s)=h(s)=\psi(s) .
$$


Remark. This result follows from Theorem 6.6 in [JOPS]. The general arguments of [JOPS] do not simplify in the special case considered here and, to avoid a complete repetition, we refer the reader to [JOPS] for details of the proof. We also remark that the arguments of [JOPS] give that the estimate $\bar{h}(s) \leq \psi(s)$ holds without any assumption on $e(\alpha)$. In analogy with Theorem 2.12, one can also prove a suitable upper bound assuming only that $e(\alpha)$ is finite in $[0,1]$. We leave the details to interested reader.

\section{Level I: Proofs.}

We start with some preliminaries. We first recall the well known

Lemma 3.1 Let $\left\{a_{t}\right\}_{t \geq 1}$ be a sequence of real numbers such that for some real number $c$ and all integers $t, s \geq 1$,

$$
a_{t+s} \leq a_{t}+a_{s}+c .
$$

Then,

$$
\lim _{t \rightarrow \infty} \frac{a_{t}}{t}=\inf _{t \geq 1} \frac{a_{t}+c}{t}
$$

Remark. Lemma 3.1 is a straightforward extension of the classical Fekete lemma [PS, Part I, Chapter 3] which states that a subadditive sequence $b_{t+s} \leq b_{t}+b_{s}$ satisfies

$$
\lim _{t \rightarrow \infty} \frac{b_{t}}{t}=\inf _{t \geq 1} \frac{b_{t}}{t}
$$

Indeed, it suffices to consider the sequence $b_{t}=a_{t}+c$. Obviously, if the subadditivity assumption (3.44) is replaced by super-additivity $a_{t+s} \geq a_{t}+a_{s}+c$, then

$$
\lim _{t \rightarrow \infty} \frac{a_{t}}{t}=\sup _{t \geq 1} \frac{a_{t}+c}{t}
$$

As already discussed in Section 2.4, one celebrated application of Fekete's Lemma concerns the existence of the Kolmogorov-Sinai entropy. The Shannon-McMillan-Breiman theorem is a deep refinement of this existence result.

Theorem 3.2 For $\mathbb{Q} \in \mathcal{P}_{\phi}$, set $S_{T}(\omega)=-\log \mathbb{Q}_{T}(\omega)$. Then the limit

$$
s(\omega)=\lim _{T \rightarrow \infty} \frac{1}{T} S_{T}(\omega)
$$

exists and satisfies $s \circ \phi(\omega)=s(\omega)$ for $\mathbb{Q}$-a.e. $\omega \in \Omega$. Moreover, one has $\mathbb{Q}[s]=h_{\phi}(\mathbb{Q})$ and

$$
\lim _{T \rightarrow \infty} \mathbb{Q}\left[\left|\frac{1}{T} S_{T}-s\right|\right]=0
$$

The Kingman subadditive ergodic theorem is a deep refinement of the Fekete Lemma. 
Theorem 3.3 Let $\mathbb{Q} \in \mathcal{P}_{\phi}$ and let $X_{T}: \Omega \rightarrow \mathbb{R}$ be a sequence of random variables such that $\mathbb{Q}\left[X_{1}^{+}\right]<\infty^{17}$. Suppose further that for some real number $C$, all integers $T, T^{\prime} \geq 1$, and $\mathbb{Q}$ a.e. $\omega \in \Omega$,

$$
X_{T+T^{\prime}}(\omega) \leq X_{T}(\omega)+X_{T^{\prime}} \circ \phi^{T}(\omega)+C .
$$

Then, the limit

$$
\lim _{T \rightarrow \infty} \frac{1}{T} X_{T}(\omega)=x(\omega)
$$

exists and satisfies $x \circ \phi(\omega)=x(\omega)$ for $\mathbb{Q}$-a.e. $\omega \in \Omega$. Moreover, one has $\mathbb{Q}\left[x^{+}\right]<\infty$, and

$$
\mathbb{Q}[x]=\lim _{T \rightarrow \infty} \frac{1}{T} \mathbb{Q}\left[X_{T}\right] \in[-\infty, \infty[.
$$

Finally, if $\mathbb{Q}[|x|]<\infty$, then

$$
\lim _{T \rightarrow \infty} \mathbb{Q}\left[\left|\frac{1}{T} X_{T}-x\right|\right]=0
$$

Remark. The Shannon-McMillan-Breiman theorem cannot be directly deduced from Theorem 3.3. However, an extension of the Kingman subadditive ergodic theorem due to Derriennic [Der] allows for such a deduction; see [BaY, Jak] for a pedagogical exposition of the proof.

The following subadditivity estimate plays a central role in our arguments. In what follows we set $\lambda_{0}=\min \operatorname{sp}(\rho)$, so that $\lambda_{0}^{-1} \rho \geq \mathbb{1}$. Recall that $\mathbb{P}_{T}^{\#}$ denotes either $\mathbb{P}_{T}$ or $\widehat{\mathbb{P}}_{T}$.

Lemma 3.4 For all $T, T^{\prime} \geq 1$,

$$
\mathbb{P}_{T+T^{\prime}}^{\#} \leq \lambda_{0}^{-1} \mathbb{P}_{T}^{\#} \mathbb{P}_{T^{\prime}}^{\#} \circ \phi^{T}
$$

Proof. It suffices to consider the case $\mathbb{P}_{T}^{\#}=\mathbb{P}_{T}$. Using the fact that $\operatorname{tr}(A B) \leq \operatorname{tr}(A)\|B\|$ for $A, B \geq 0$, we can write

$$
\begin{aligned}
\mathbb{P}_{T+T^{\prime}}\left(\omega_{1}, \ldots, \omega_{T+T^{\prime}}\right) & =\operatorname{tr}\left(\rho\left(\Phi_{\omega_{1}} \circ \cdots \circ \Phi_{\omega_{T+T^{\prime}}}\right)[\mathbb{1}]\right) \\
& =\operatorname{tr}\left(\left(\Phi_{\omega_{T}}^{*} \circ \cdots \circ \Phi_{\omega_{1}}^{*}\right)[\rho]\left(\Phi_{\omega_{T+1}} \circ \cdots \circ \Phi_{\omega_{T+T^{\prime}}}\right)[\mathbb{1}]\right) \\
& \leq \operatorname{tr}\left(\left(\Phi_{\omega_{T}}^{*} \circ \cdots \circ \Phi_{\omega_{1}}^{*}\right)[\rho]\right)\left\|\left(\Phi_{\omega_{T+1}} \circ \cdots \circ \Phi_{\omega_{T+T^{\prime}}}\right)[\mathbb{1}]\right\| .
\end{aligned}
$$

The estimate

$$
\left\|\left(\Phi_{\omega_{T+1}} \circ \cdots \circ \Phi_{\omega_{T+T^{\prime}}}\right)[\mathbb{1}]\right\| \leq \operatorname{tr}\left(\left(\Phi_{\omega_{T+1}} \circ \cdots \circ \Phi_{\omega_{T+T^{\prime}}}\right)[\mathbb{1}]\right) \leq \lambda_{0}^{-1} \operatorname{tr}\left(\rho\left(\Phi_{\omega_{T+1}} \circ \cdots \circ \Phi_{\omega_{T+T^{\prime}}}\right)[\mathbb{1}]\right)
$$

thus leads to

$$
\begin{aligned}
\mathbb{P}_{T+T^{\prime}}\left(\omega_{1}, \ldots, \omega_{T+T^{\prime}}\right) & \leq \lambda_{0}^{-1} \operatorname{tr}\left(\rho\left(\Phi_{\omega_{T}} \circ \cdots \circ \Phi_{\omega_{1}}\right)[\mathbb{1}]\right) \operatorname{tr}\left(\rho\left(\Phi_{\omega_{T+1}} \circ \cdots \circ \Phi_{\omega_{T+T^{\prime}}}\right)[\mathbb{1}]\right) \\
& =\lambda_{0}^{-1} \mathbb{P}_{T}\left(\omega_{1}, \ldots, \omega_{T}\right) \mathbb{P}_{T^{\prime}}\left(\omega_{T+1}, \ldots, \omega_{T+T^{\prime}}\right) .
\end{aligned}
$$

\footnotetext{
${ }^{17} X_{1}^{+}=\max \left(X_{1}, 0\right)$.
} 


\subsection{Proof of Theorem 2.1}

Parts (1-2) Writing

$$
\mathbb{E}\left[\sigma_{T}\right]=S\left(\mathbb{P}_{T} \mid \widehat{\mathbb{P}}_{T}\right)=-S\left(\mathbb{P}_{T}\right)-\sum_{\omega \in \Omega_{T}} \mathbb{P}_{T}(\omega) \log \widehat{\mathbb{P}}_{T}(\omega),
$$

the subadditivity of entropy (2.4) and Lemma 3.4 give

$$
\mathbb{E}\left[\sigma_{T+T^{\prime}}\right] \geq \mathbb{E}\left[\sigma_{T}\right]+\mathbb{E}\left[\sigma_{T^{\prime}}\right]+\log \lambda_{0}
$$

Fekete's Lemma and the right inequality in (2.15) yield the results.

Part (3) Note that

$$
\sigma_{T}(\omega)=-X_{T}(\omega)-Y_{T}(\omega)
$$

where $X_{T}(\omega)=-\log \mathbb{P}_{T}(\omega) \geq 0$ and $Y_{T}(\omega)=\log \widehat{\mathbb{P}}_{T}(\omega) \leq 0$.

Applying the Shannon-McMillan-Breiman theorem to $X_{T}$, we conclude that the limit

$$
x(\omega)=\lim _{T \rightarrow \infty} \frac{1}{T} X_{T}(\omega)
$$

exists $\mathbb{P}$-a.s. and in $L^{1}(\Omega, d \mathbb{P})$, is $\phi$-invariant and non-negative.

Since $Y_{T} \leq 0$, and Lemma 3.4 implies that $Y_{T+T^{\prime}} \leq Y_{T}+Y_{T^{\prime}} \circ \phi^{T}-\log \lambda_{0}$, we can apply Kingman's subadditive ergodic theorem to conclude that the limit

$$
y(\omega)=\lim _{T \rightarrow \infty} \frac{1}{T} Y_{T}(\omega)
$$

exists $\mathbb{P}$-a.s., is $\phi$-invariant, non-positive, and satisfies

$$
\mathbb{E}[y]=\lim _{T \rightarrow \infty} \frac{1}{T} \mathbb{E}\left[Y_{T}\right]
$$

It follows that (2.16) holds $\mathbb{P}$-a.s. with $\bar{\sigma}=-x-y$. Since both $x$ and $y$ are $\phi$-invariant, so is $\bar{\sigma}$. The $L^{1}$-convergence in (3.45) together with (3.47) yield that

$$
\mathbb{E}[\bar{\sigma}]=\lim _{T \rightarrow \infty} \frac{1}{T} \mathbb{E}\left[\sigma_{T}\right]=\operatorname{ep}(\mathcal{J}, \rho)
$$

From the fact that $\bar{\sigma}_{-} \leq x$ we further deduce that $\mathbb{E}\left[\bar{\sigma}_{-}\right] \leq \mathbb{E}[x]=h_{\phi}(\mathbb{P})<\infty$. Finally, if $\operatorname{ep}(\mathcal{J}, \rho)<\infty$, then

$$
\mathbb{E}[|\bar{\sigma}|]=\mathbb{E}\left[\bar{\sigma}+2 \bar{\sigma}_{-}\right] \leq 2 h_{\phi}(\mathbb{P})+\operatorname{ep}(\mathcal{J}, \rho)<\infty
$$

and Kingman's ergodic theorem implies that (3.46) holds in $L^{1}(\Omega, d \mathbb{P})$. Thus, (2.16) also holds in $L^{1}(\Omega, d \mathbb{P})$. 


\subsection{Proof of Proposition 2.2}

The first statement clearly follows from the fact that $\mathbb{P}=\widehat{\mathbb{P}}$ implies $\sigma_{T}=0$. For all $T \geq 1$, set

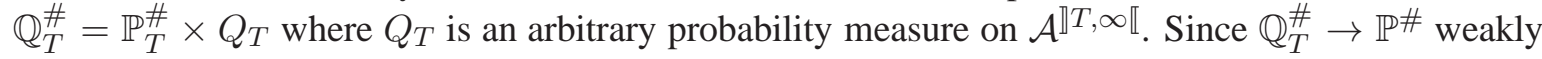
as $T \rightarrow \infty$, the lower semicontinuity of the relative entropy gives

$$
S(\mathbb{P} \mid \widehat{\mathbb{P}}) \leq \liminf _{T \rightarrow \infty} S\left(\mathbb{Q}_{T} \mid \widehat{\mathbb{Q}}_{T}\right) .
$$

The obvious relation $S\left(\mathbb{Q}_{T} \mid \widehat{\mathbb{Q}}_{T}\right)=S\left(\mathbb{P}_{T} \mid \widehat{\mathbb{P}}_{T}\right)$ and Eq. (2.14) further yield

$$
S\left(\mathbb{Q}_{T} \mid \widehat{\mathbb{Q}}_{T}\right)=\mathbb{E}\left[\sigma_{T}\right]
$$

In view of our assumption, Theorem 2.1 (2) writes

$$
\mathbb{E}\left[\sigma_{T}\right]+\log \lambda_{0} \leq \operatorname{ep}(\mathcal{J}, \rho) T=0,
$$

for all $T \geq 1$. Thus, it follows from (2.15) that

$$
\lim _{T \rightarrow \infty} \mathbb{E}\left[\sigma_{T}\right]=\sup _{T \geq 1} \mathbb{E}\left[\sigma_{T}\right] \leq \log \lambda_{0}^{-1}
$$

which proves Part (1). Combining the last estimate with (3.48) and (3.49) we further get

$$
S(\mathbb{P} \mid \widehat{\mathbb{P}}) \leq \log \lambda_{0}^{-1} .
$$

In the same way one derives that $S(\widehat{\mathbb{P}} \mid \mathbb{P}) \leq \log \lambda_{0}^{-1}$ and Part (2) follows. Finally, assuming that $\mathbb{P}$ is $\phi$-ergodic, Part (3) follows from the facts that $\widehat{\mathbb{P}} \in \mathcal{P}_{\phi}$ and $\widehat{\mathbb{P}} \ll \mathbb{P}$.

\subsection{Proof of Theorem 2.3}

For a given $\delta>0$ let $\mathcal{T}_{T, \delta}=\left\{\omega \in \Omega_{T} \mid \sigma_{T}(\omega) \geq c_{\delta} T\right\}$, where $c_{\delta}=\delta$ whenever $\operatorname{ep}(\mathcal{J}, \rho)=\infty$ and $c_{\delta}=\operatorname{ep}(\mathcal{J}, \rho)-\delta$ otherwise. Since $\mathbb{P}$ is $\phi$-ergodic, Theorem 2.1 (3) yields

$$
\lim _{T \rightarrow \infty} \mathbb{P}_{T}\left(\mathcal{T}_{T, \delta}\right)=1
$$

Thus, for $T$ large enough, $\mathbb{P}_{T}\left(\mathcal{T}_{T, \delta}^{c}\right) \leq \epsilon$ and consequently $s_{T}(\epsilon) \leq \widehat{\mathbb{P}}_{T}\left(\mathcal{T}_{T, \delta}\right)$. We also have

$$
\widehat{\mathbb{P}}_{T}\left(\mathcal{T}_{T, \delta}\right)=\widehat{\mathbb{P}}_{T}\left(\left\{\omega \in \Omega_{T} \mid 1 \leq \mathrm{e}^{\sigma_{T}(\omega)-c_{\delta} T}\right\}\right) \leq \mathrm{e}^{-c_{\delta} T} \sum_{\omega \in \Omega_{T}} \mathrm{e}^{\sigma_{T}(\omega)} \widehat{\mathbb{P}}_{T}(\omega)=\mathrm{e}^{-c_{\delta} T}
$$

Hence, for any $\delta>0$,

$$
\limsup _{T \rightarrow \infty} \frac{1}{T} \log s_{T}(\epsilon) \leq-c_{\delta}
$$

Taking $\delta \downarrow 0$ in the case ep $(\mathcal{J}, \rho)<\infty$ and $\delta \uparrow \infty$ in the opposite case gives the upper bound

$$
\limsup _{T \rightarrow \infty} \frac{1}{T} \log s_{T}(\epsilon) \leq-\operatorname{ep}(\mathcal{J}, \rho) .
$$


To prove the lower bound we may assume that $\operatorname{ep}(\mathcal{J}, \rho)<\infty$. Let $\mathcal{U}_{T, \epsilon}$ be a subset of $\Omega_{T}$ for which the minimum in Eq. (2.19) is achieved, i.e., $s_{T}(\epsilon)=\widehat{\mathbb{P}}_{T}\left(\mathcal{U}_{T, \epsilon}\right)$ and $\mathbb{P}_{T}\left(\mathcal{U}_{T, \epsilon}^{c}\right) \leq \epsilon$. For a given $\delta>0$ let $\mathcal{D}_{T, \delta}=\left\{\omega \in \Omega_{T} \mid \sigma_{T}(\omega) \leq(\operatorname{ep}(\mathcal{J}, \rho)+\delta) T\right\}$. Invoking Theorem 2.1 (3) again gives

$$
\lim _{T \rightarrow \infty} \mathbb{P}_{T}\left(\mathcal{D}_{T, \delta}\right)=1
$$

and so, for $T$ large enough, $\mathbb{P}_{T}\left(\mathcal{D}_{T, \delta}^{c}\right) \leq \frac{1}{2}(1-\epsilon)$. Since $\mathbb{P}_{T}\left(\mathcal{U}_{T, \epsilon}^{c}\right) \leq \epsilon$, we then have

$$
\begin{aligned}
\frac{1}{2}(1-\epsilon) & \leq 1-\left(\mathbb{P}_{T}\left(\mathcal{U}_{T, \epsilon}^{c}\right)+\mathbb{P}_{T}\left(\mathcal{D}_{T, \delta}^{c}\right)\right) \leq \mathbb{P}_{T}\left(\mathcal{U}_{T, \epsilon} \cap \mathcal{D}_{T, \delta}\right)=\int_{\mathcal{U}_{T, \epsilon} \cap \mathcal{D}_{T, \delta}} \mathrm{e}^{\sigma_{T}} \mathrm{~d} \widehat{\mathbb{P}}_{T} \\
& \leq \mathrm{e}^{T(\operatorname{ep}(\mathcal{J}, \rho)+\delta)} \widehat{\mathbb{P}}_{T}\left(\mathcal{U}_{T, \epsilon} \cap \mathcal{D}_{T, \delta}\right) \leq \mathrm{e}^{T(\operatorname{ep}(\mathcal{J}, \rho)+\delta)} \widehat{\mathbb{P}}_{T}\left(\mathcal{U}_{T, \epsilon}\right),
\end{aligned}
$$

and hence

$$
s_{T}(\epsilon)=\widehat{\mathbb{P}}_{T}\left(\mathcal{U}_{T, \epsilon}\right) \geq \frac{1}{2}(1-\epsilon) \mathrm{e}^{-T(\operatorname{ep}(\mathcal{J}, \rho)+\delta)} .
$$

It follows that for any $\delta>0$,

$$
\liminf _{T \rightarrow \infty} \frac{1}{T} s_{T}(\epsilon) \geq-\operatorname{ep}(\mathcal{J}, \rho)-\delta
$$

so that

$$
\liminf _{T \rightarrow \infty} \frac{1}{T} s_{T}(\epsilon) \geq-\operatorname{ep}(\mathcal{J}, \rho) .
$$

The result follows by combining the obtained lower and upper bounds.

Remark. The assertions of Remark 2 after Theorem 2.3 can be deduced from the above arguments as follows. Let $\left\{\mathcal{T}_{T}\right\}_{T \geq 1}$ be a sequence such that $\mathcal{T}_{T} \subset \Omega_{T}$ and $\mathbb{P}_{T}\left(\mathcal{T}_{T}^{c}\right) \rightarrow 0$ as $T \rightarrow \infty$ and fix $\epsilon \in] 0,1\left[\right.$. For large enough $T$ one has $\mathbb{P}_{T}\left(\mathcal{T}_{T}^{c}\right) \leq \epsilon$ and hence $\widehat{\mathbb{P}}_{T}\left(\mathcal{T}_{T}\right) \geq s_{T}(\epsilon)$. It follows from Theorem 2.3 that

$$
-\operatorname{ep}(\mathcal{J}, \rho) \leq \underline{s} \leq \bar{s}
$$

Reciprocally, Eq. (3.50) shows that the above tests $\mathcal{T}_{T, \delta}$ are such that $\mathbb{P}_{T}\left(\mathcal{T}_{T, \delta}^{c}\right) \rightarrow 0$ as $T \rightarrow \infty$ and taking $\delta$ to $0 / \infty$ in Eq. (3.51) as above yields $\bar{s} \leq-\operatorname{ep}(\mathcal{J}, \rho)$.

\section{Level II: Proofs.}

\subsection{Proof of Theorem 2.4}

Parts (1)-(2) By Definition (2.21) and Lemma 3.4, we have

$$
\begin{aligned}
e_{T+T^{\prime}}(\alpha) & =\log \left(\sum_{\omega \in \Omega_{T+T^{\prime}}} \mathbb{P}_{T+T^{\prime}}(\omega)^{1-\alpha} \widehat{\mathbb{P}}_{T+T^{\prime}}(\omega)^{\alpha}\right) \\
& \leq \log \left(\sum_{\omega \in \Omega_{T+T^{\prime}}}\left(\lambda_{0}^{-1} \mathbb{P}_{T}(\omega) \mathbb{P}_{T^{\prime}} \circ \phi^{T}(\omega)\right)^{1-\alpha}\left(\lambda_{0}^{-1} \widehat{\mathbb{P}}_{T}(\omega) \widehat{\mathbb{P}}_{T^{\prime}} \circ \phi^{T}(\omega)\right)^{\alpha}\right) \\
& =\log \left(\lambda_{0}^{-1} \sum_{\omega \in \Omega_{T}} \mathbb{P}_{T}(\omega)^{1-\alpha} \widehat{\mathbb{P}}_{T}(\omega)^{\alpha} \sum_{\omega^{\prime} \in \Omega_{T^{\prime}}} \mathbb{P}_{T^{\prime}}\left(\omega^{\prime}\right)^{1-\alpha} \widehat{\mathbb{P}}_{T^{\prime}}\left(\omega^{\prime}\right)^{\alpha}\right) \\
& =e_{T}(\alpha)+e_{T^{\prime}}(\alpha)+\log \lambda_{0}^{-1},
\end{aligned}
$$


On entropy production of repeated quantum measurements I

for all $T, T^{\prime} \geq 1$ and all $\alpha \in[0,1]$. Thus, the existence of the limit $e(\alpha)$ is a consequence of the Fekete lemma which gives

$$
e(\alpha)=\inf _{T \geq 1} \frac{1}{T}\left(e_{T}(\alpha)+\log \lambda_{0}^{-1}\right) .
$$

The function $[0,1] \ni \alpha \mapsto e(\alpha)$ is convex as a limit of convex functions and upper semicontinuous as the infimum of the continuous functions. The symmetry (2.26) and the values of $e(0)$ and $e(1)$ follow from the respective properties of $e_{T}(\alpha)$. Part (2) follows from the corresponding property of the function $\bar{e}$ established in the introductory discussion of Section 2.5.

Part (3) By Lemma 3.4, one has

$$
\mathbb{Q}\left[-\log \mathbb{P}_{T+T^{\prime}}\right] \geq \mathbb{Q}\left[-\log \mathbb{P}_{T}\right]+\mathbb{Q}\left[-\log \mathbb{P}_{T^{\prime}} \circ \phi^{T}\right]+\log \lambda_{0}
$$

The $\phi$-invariance of $\mathbb{Q}$ and Fekete's lemma imply

$$
\varsigma(\mathbb{Q})=\lim _{T \rightarrow \infty} \mathbb{Q}\left[-\frac{1}{T} \log \mathbb{P}_{T}\right]=\sup _{T \geq 1} \frac{1}{T}\left(\mathbb{Q}\left[-\log \mathbb{P}_{T}\right]+\log \lambda_{0}\right) .
$$

Since $\mathbb{Q}\left[-\log \mathbb{P}_{T}\right] \geq 0$, the limit $\varsigma(\mathbb{Q})$ takes value in $[0,+\infty]$ (the limit is $+\infty$, for example, in the cases where $\operatorname{supp} \mathbb{Q}_{T} \not \subset \operatorname{supp} \mathbb{P}_{T}$ for some $T$ ). The function $\mathbb{Q} \mapsto \varsigma(\mathbb{Q})$ is affine as a limit of affine functions and lower semicontinuous as supremum of continuous functions. The last assertion follows from Eq. (2.20).

Part (4) Since the maps $\mathcal{P}_{\phi} \ni \mathbb{Q} \mapsto h_{\phi}(\mathbb{Q})$ and $\mathcal{P}_{\phi} \ni \mathbb{Q} \mapsto-\varsigma(\mathbb{Q})$ are both affine and upper semicontinuous, so is $\mathcal{P}_{\phi} \ni \mathbb{Q} \mapsto f(\mathbb{Q})$. The last assertion of Part (3) gives $f(\mathbb{P})=0$. From the variational inequality (2.24) we deduce that for all $T \geq 1$, all $\alpha \in \mathbb{R}$ and all $\mathbb{Q} \in \mathcal{P}_{\phi}$,

$$
\frac{1}{T} e_{T}(\alpha) \geq \frac{1}{T} \mathbb{Q}^{(\alpha)}\left[\log \mathbb{P}_{T}\right]-\frac{1}{T} \mathbb{Q}\left[\log \mathbb{Q}_{T}\right] .
$$

Taking the limit $T \rightarrow \infty$ on both sides of this inequality yields the last assertion.

Part (5) For $\alpha \in[0,1]$, the map $\mathcal{P}_{\phi} \ni \mathbb{Q} \mapsto \mathbb{Q}^{(\alpha)} \in \mathcal{P}_{\phi}$ is affine and continuous. Thus, we deduce from Part (4) that $\mathcal{P}_{\phi} \ni \mathbb{Q} \mapsto f\left(\mathbb{Q}^{(\alpha)}\right)$ is affine and upper semicontinuous. These two properties respectively imply that $\mathcal{P}_{\text {eq }}(\alpha)$ is convex and compact. Let $m$ be a probability measure on $\mathcal{P}_{\phi}$ such that $\int \mathbb{Q} \mathrm{d} m(\mathbb{Q})=\overline{\mathbb{Q}} \in \mathcal{P}_{\text {eq }}(\alpha)$. It follows that $\overline{\mathbb{Q}}^{(\alpha)}=\int \mathbb{Q}^{(\alpha)} \mathrm{d} m(\mathbb{Q})$ and since $f$ is affine and upper semicontinuous

$$
0=e(\alpha)-f\left(\overline{\mathbb{Q}}^{(\alpha)}\right)=\int\left(e(\alpha)-f\left(\mathbb{Q}^{(\alpha)}\right)\right) \mathrm{d} m(\mathbb{Q}),
$$

from which we conclude that $m$ is concentrated on $\mathcal{P}_{\text {eq }}(\alpha)$, and hence that $\mathcal{P}_{\text {eq }}(\alpha)$ is a face of $\mathcal{P}_{\phi}$. In particular, the extreme points of $\mathcal{P}_{\text {eq }}(\alpha)$ are $\phi$-ergodic; see [Ru1, Section A.5.6].

It remains to prove that $\mathcal{P}_{\text {eq }}(\alpha)$ is non-empty. Since $\mathbb{P}^{(0)}=\mathbb{P}$ and $f(\mathbb{P})=0=e(0)$, one has $\mathbb{P} \in \mathcal{P}_{\text {eq }}(0)$. From the fact that $\mathbb{Q}^{(1-\alpha)}=\widehat{\mathbb{Q}}^{(\alpha)}$ we further deduce that $\widehat{\mathbb{P}} \in \mathcal{P}_{\text {eq }}(1)$. Thus, we may assume that $\alpha \in] 0,1[$.

For $T \geq 1$, the measure $Q_{T} \in \mathcal{P}_{\Omega_{T}}$ defined by

$$
Q_{T}(\omega)=\mathrm{e}^{-e_{T}(\alpha)} \mathbb{P}_{T}(\omega)^{1-\alpha} \widehat{\mathbb{P}}_{T}(\omega)^{\alpha}
$$

achieves the maximum on the first line of (2.24),

$$
e_{T}(\alpha)=S\left(Q_{T}\right)+(1-\alpha) Q_{T}\left[\log \mathbb{P}_{T}\right]+\alpha Q_{T}\left[\log \widehat{\mathbb{P}}_{T}\right] .
$$


Decomposing $\Omega=\Omega_{T}^{\mathbb{N}}$ into the product of blocks of size $T$, we extend $Q_{T}$ to a product probability measure $\mathbb{Q}_{[T]}=Q_{T}^{\times \mathbb{N}} \in \mathcal{P}_{\phi^{T}}$. Setting

$$
\mathbb{Q}_{(T)}=\frac{1}{T} \sum_{t=0}^{T-1} \mathbb{Q}_{[T]} \circ \phi^{-t}
$$

and observing that

$$
\mathbb{Q}_{(T)}=\frac{1}{2 T} \sum_{t=0}^{2 T-1} \mathbb{Q}_{[T]} \circ \phi^{-t}
$$

we obtain

$$
\mathbb{Q}_{(T)} \circ \phi^{-1}=\frac{1}{2 T} \sum_{t=1}^{2 T} \mathbb{Q}_{[T]} \circ \phi^{-t}=\mathbb{Q}_{(T)}-\frac{1}{2 T}\left(\mathbb{Q}_{[T]}-\mathbb{Q}_{[T]} \circ \phi^{-2 T}\right)=\mathbb{Q}_{(T)},
$$

so that $\mathbb{Q}_{(T)} \in \mathcal{P}_{\phi}$. Invoking a well known property of the Kolmogorov-Sinai entropy (see, e.g., [Wa, Theorem 4.13]), its affine property and the Kolmogorov-Sinai theorem, we can write

$$
h_{\phi}\left(\mathbb{Q}_{(T)}\right)=\frac{1}{T} h_{\phi^{T}}\left(\mathbb{Q}_{(T)}\right)=\frac{1}{T^{2}} \sum_{t=0}^{T-1} h_{\phi^{T}}\left(\mathbb{Q}_{[T]} \circ \phi^{-t}\right)=\frac{1}{T^{2}} \sum_{t=0}^{T-1} \mathbb{Q}_{[T]}\left[-\log Q_{T} \circ \phi^{t}\right]
$$

For $t \in \llbracket 0, T-1 \rrbracket$, we derive a lower bound for $\mathbb{Q}_{[T]}\left[-\log Q_{T} \circ \phi^{t}\right]$ in the following way (we denote by $\boldsymbol{\omega} \boldsymbol{\nu} \in \Omega_{T+T^{\prime}}$ the word obtained by juxtaposition of the two words $\boldsymbol{\omega} \in \Omega_{T}$ and $\boldsymbol{\nu} \in \Omega_{T^{\prime}}$ )

$$
\begin{aligned}
\mathbb{Q}_{[T]}\left[-\log Q_{T} \circ \phi^{t}\right] & =-\sum_{\substack{\boldsymbol{\xi}, \boldsymbol{\nu} \in \Omega_{t} \\
\boldsymbol{\omega}, \boldsymbol{\eta} \in \Omega_{T-t}}} Q_{T}(\boldsymbol{\xi} \boldsymbol{\omega}) Q_{T}(\boldsymbol{\nu} \boldsymbol{\eta}) \log Q_{T}(\boldsymbol{\omega} \boldsymbol{\nu}) \\
& =-\frac{1}{2} \sum_{\substack{\boldsymbol{\xi}, \boldsymbol{\nu} \in \Omega_{t} \\
\boldsymbol{\omega}, \boldsymbol{\eta} \in \Omega_{T-t}}} Q_{T}(\boldsymbol{\xi} \boldsymbol{\omega}) Q_{T}(\boldsymbol{\nu} \boldsymbol{\eta}) \log \left(Q_{T}(\boldsymbol{\omega} \boldsymbol{\nu}) Q_{T}(\boldsymbol{\eta} \boldsymbol{\xi})\right) \\
& =\frac{1}{2} \sum_{\substack{\boldsymbol{\xi}, \boldsymbol{\nu} \in \Omega_{t} \\
\boldsymbol{\omega}, \boldsymbol{\eta} \in \Omega_{T-t}}} Q_{T}(\boldsymbol{\xi} \boldsymbol{\omega}) Q_{T}(\boldsymbol{\nu} \boldsymbol{\eta})\left[\log \frac{Q_{T}(\boldsymbol{\xi} \boldsymbol{\omega}) Q_{T}(\boldsymbol{\nu} \boldsymbol{\eta})}{Q_{T}(\boldsymbol{\omega} \boldsymbol{\nu}) Q_{T}(\boldsymbol{\eta} \boldsymbol{\xi})}-\log Q_{T}(\boldsymbol{\xi} \boldsymbol{\omega}) Q_{T}(\boldsymbol{\nu} \boldsymbol{\eta})\right] \\
& \geq \frac{1}{2} S\left(Q_{T} \times Q_{T}\right)=S\left(Q_{T}\right),
\end{aligned}
$$

where, in the last line, we have used the non-negativity of relative entropy. Combining this lower bound with (4.55) we obtain

$$
h_{\phi}\left(\mathbb{Q}_{(T)}\right) \geq \frac{1}{T^{2}} \sum_{t=0}^{T-1} S\left(Q_{T}\right)=\frac{1}{T} S\left(Q_{T}\right),
$$

and (4.54) yields

$$
\frac{1}{T} e_{T}(\alpha) \leq h_{\phi}\left(\mathbb{Q}_{(T)}\right)+(1-\alpha) Q_{T}\left[\frac{1}{T} \log \mathbb{P}_{T}\right]+\alpha Q_{T}\left[\frac{1}{T} \log \widehat{\mathbb{P}}_{T}\right]
$$

By the compactness of $\mathcal{P}_{\phi}$, there exists a sequence $T_{k} \uparrow \infty$ such that $\lim _{k \rightarrow \infty} \mathbb{Q}_{\left(T_{k}\right)}=\mathbb{Q} \in \mathcal{P}_{\phi}$. Applying Lemma 2.3 in [CFH] to the sequence $f_{T}=\lambda_{0}^{-1} \mathbb{P}_{T}^{\#}$ we derive

$$
\limsup _{k \rightarrow \infty} Q_{T_{k}}\left[\frac{1}{T_{k}} \log \mathbb{P}_{T_{k}}^{\#}\right] \leq \lim _{T \rightarrow \infty} \mathbb{Q}\left[\frac{1}{T} \log \mathbb{P}_{T}^{\#}\right]
$$


Combining these two inequalities with Relation (4.56) and Parts (1) and (3) gives

$$
e(\alpha) \leq \limsup _{k \rightarrow \infty} h_{\phi}\left(\mathbb{Q}_{\left(T_{k}\right)}\right)-\varsigma\left(\mathbb{Q}^{(\alpha)}\right) .
$$

Finally, invoking the the upper semicontinuity of the Kolmogorov-Sinai entropy we derive

$$
e(\alpha) \leq h_{\phi}(\mathbb{Q})-\varsigma\left(\mathbb{Q}^{(\alpha)}\right)=f\left(\mathbb{Q}^{(\alpha)}\right),
$$

and so $\mathbb{Q} \in \mathcal{P}_{\text {eq }}(\alpha)$.

Part (6) We follow the proof of [Fe1, Theorem 1.2] and start by recalling the basic properties of the function $[0,1] \ni \alpha \mapsto e(\alpha)$ which follow from the fact that it is convex and finite; see [Ro].

(i) $\alpha \mapsto e(\alpha)$ is continuous.

(ii) For each $\alpha \in] 0,1]$ the left derivative $\partial^{-} e(\alpha)$ exists.

(iii) For each $\alpha \in\left[0,1\left[\right.\right.$ the right derivative $\partial^{+} e(\alpha)$ exists.

(iv) $\partial^{+} e(0) \leq \partial^{-} e(\alpha) \leq \partial^{+} e(\alpha) \leq \partial^{-} e\left(\alpha^{\prime}\right) \leq \partial^{+} e\left(\alpha^{\prime}\right) \leq \partial^{-} e(1)$ for $0 \leq \alpha \leq \alpha^{\prime} \leq 1$.

(v) There is an at most countable subset $C \subset[0,1]$ such that $\partial^{-} e(\alpha)=\partial^{+} e(\alpha)=e^{\prime}(\alpha)$ holds for all $\alpha \in[0,1] \backslash C$.

(vi) For any $\left.\left.\alpha_{0} \in\right] 0,1\right], \lim _{\alpha \uparrow \alpha_{0}} \partial^{+} e(\alpha)=\lim _{\alpha \uparrow \alpha_{0}} \partial^{-} e(\alpha)=\partial^{-} e\left(\alpha_{0}\right)$. For any $\alpha_{0} \in[0,1[$, $\lim _{\alpha \downarrow \alpha_{0}} \partial^{+} e(\alpha)=\lim _{\alpha \downarrow \alpha_{0}} \partial^{-} e(\alpha)=\partial^{+} e\left(\alpha_{0}\right)$.

Fix $\alpha \in] 0,1\left[\right.$. For any $\mathbb{Q} \in \mathcal{P}_{\text {eq }}(\alpha)$ one has $\varsigma(\mathbb{Q})<\infty$, and for $\epsilon>0$ small enough,

$$
e(\alpha+\epsilon) \geq f\left(\mathbb{Q}^{(\alpha+\epsilon)}\right)=f\left(\mathbb{Q}^{(\alpha)}\right)+\epsilon(\varsigma(\mathbb{Q})-\varsigma(\widehat{\mathbb{Q}}))=e(\alpha)+\epsilon(\varsigma(\mathbb{Q})-\varsigma(\widehat{\mathbb{Q}})) .
$$

This gives that $\partial^{+} e(\alpha) \geq \varsigma(\mathbb{Q})-\varsigma(\widehat{\mathbb{Q}})$, and so

$$
\partial^{+} e(\alpha) \geq \sup _{\mathbb{Q} \in \mathcal{P}_{\mathrm{eq}}(\alpha)}(\varsigma(\mathbb{Q})-\varsigma(\widehat{\mathbb{Q}}))
$$

In the same way one derives

$$
\partial^{-} e(\alpha) \leq \inf _{\mathbb{Q} \in \mathcal{P}_{\mathrm{eq}}(\alpha)}(\varsigma(\mathbb{Q})-\varsigma(\widehat{\mathbb{Q}}))
$$

In particular, if $e^{\prime}(\alpha)$ exists, then

$$
e^{\prime}(\alpha)=\varsigma(\mathbb{Q})-\varsigma(\widehat{\mathbb{Q}})
$$

for all $\mathbb{Q} \in \mathcal{P}_{\text {eq }}(\alpha)$.

Let now $\left.\alpha_{k} \in\right] 0,1\left[\backslash C\right.$ be a sequence such that $\alpha_{k} \downarrow \alpha$. By Properties (v) and (vi) $e^{\prime}\left(\alpha_{k}\right)$ exists, and

$$
\lim _{k \rightarrow \infty} e^{\prime}\left(\alpha_{k}\right)=\partial^{+} e(\alpha)
$$

Choose $\mathbb{Q}_{k} \in \mathcal{P}_{\text {eq }}\left(\alpha_{k}\right)$. Passing to a subsequence, which we also denote by $\alpha_{k}$, we may assume that $\lim _{k \rightarrow \infty} \mathbb{Q}_{k}=\mathbb{Q} \in \mathcal{P}_{\phi}$. Property (i) and the upper semicontinuity of $f$ imply

$$
e(\alpha)=\lim _{k \rightarrow \infty} e\left(\alpha_{k}\right)=\lim _{k \rightarrow \infty} f\left(\mathbb{Q}_{k}^{\left(\alpha_{k}\right)}\right) \leq f\left(\mathbb{Q}^{(\alpha)}\right),
$$


and we conclude that $\mathbb{Q} \in \mathcal{P}_{\text {eq }}(\alpha)$. The relation

$$
\lim _{k \rightarrow \infty}\left(h_{\phi}\left(\mathbb{Q}_{k}^{\left(\alpha_{k}\right)}\right)-\varsigma\left(\mathbb{Q}_{k}^{\left(\alpha_{k}\right)}\right)\right)=h_{\phi}\left(\mathbb{Q}^{(\alpha)}\right)-\varsigma\left(\mathbb{Q}^{(\alpha)}\right)
$$

together with the upper-semicontinuity of $h_{\phi}$ and the lower-semicontinuity of $\varsigma$ gives

$$
\liminf _{k \rightarrow \infty} \varsigma\left(\mathbb{Q}_{k}^{\left(\alpha_{k}\right)}\right)=\varsigma\left(\mathbb{Q}^{(\alpha)}\right) .
$$

Passing again to a subsequence, we may assume that

$$
\lim _{k \rightarrow \infty} \varsigma\left(\mathbb{Q}_{k}^{\left(\alpha_{k}\right)}\right)=\varsigma\left(\mathbb{Q}^{(\alpha)}\right) .
$$

The lower-semicontinuity of $\varsigma$ now gives that

$$
\liminf _{k \rightarrow \infty} \varsigma\left(\mathbb{Q}_{k}\right)=\varsigma(\mathbb{Q}), \quad \liminf _{k \rightarrow \infty} \varsigma\left(\widehat{\mathbb{Q}}_{k}\right)=\varsigma(\widehat{\mathbb{Q}}) .
$$

Passing again to a subsequence, we may assume that $\lim _{k \rightarrow \infty} \varsigma\left(\mathbb{Q}_{k}\right)=\varsigma(\mathbb{Q})$. Then (4.61) gives that along this final subsequence $\lim _{k \rightarrow \infty} \varsigma\left(\widehat{\mathbb{Q}}_{k}\right)=\varsigma(\widehat{\mathbb{Q}})$. Combining this fact with Relations (4.59) and (4.60) we derive

$$
\partial^{+} e(\alpha)=\lim _{k \rightarrow \infty} \varsigma\left(\mathbb{Q}_{k}\right)-\varsigma\left(\widehat{\mathbb{Q}}_{k}\right)=\varsigma(\mathbb{Q})-\varsigma(\widehat{\mathbb{Q}})
$$

and so, by (4.58),

$$
\partial^{+} e(\alpha)=\sup _{\mathbb{Q} \in \mathcal{P}_{\text {eq }}(\alpha)} \varsigma(\mathbb{Q})-\varsigma(\widehat{\mathbb{Q}}) .
$$

An analogous argument yields

$$
\partial^{-} e(\alpha)=\inf _{\mathbb{Q} \in \mathcal{P}_{\mathrm{eq}}(\alpha)} \varsigma(\mathbb{Q})-\varsigma(\widehat{\mathbb{Q}})
$$

Part (7) The proof of relations (2.28) follows the proof of Part (6). The inequalities follow from $\mathbb{P} \in \mathcal{P}_{\text {eq }}(0), \widehat{\mathbb{P}} \in \mathcal{P}_{\text {eq }}(1)$. The only difference is that for $\mathbb{Q} \in \mathcal{P}_{\text {eq }}(0), \varsigma(\mathbb{Q})<\infty$ while $\varsigma(\widehat{\mathbb{Q}})$ is allowed to take value $\infty$, which leads to the possibility that $\partial^{+} e(0)=-\infty$. The case $\mathbb{Q} \in \mathcal{P}_{\text {eq }}(1)$ is analogous.

Part (8) Assume that $\mathbb{P}$ is ergodic, let $\mathbb{Q} \in \mathcal{P}_{\text {eq }}(0)$ and set

$$
a_{T}=S\left(\mathbb{Q}_{T} \mid \mathbb{P}_{T}\right)=\mathbb{Q}\left[-\log \mathbb{P}_{T}\right]-S\left(\mathbb{Q}_{T}\right) .
$$

Relation (4.53) and the subadditivity of entropy give that for all $T, T^{\prime} \geq 1$,

$$
a_{T+T^{\prime}} \geq a_{T}+a_{T^{\prime}}+\log \lambda_{0}
$$

By Fekete's lemma, we have

$$
0=e(0)=f(\mathbb{Q})=\lim _{T \rightarrow \infty} \frac{a_{T}}{T}=\sup _{T \geq 1} \frac{1}{T}\left(a_{T}+\log \lambda_{0}\right)
$$

Hence, $S\left(\mathbb{Q}_{T} \mid \mathbb{P}_{T}\right) \leq \log \lambda_{0}^{-1}$ for all $T \geq 1$ and the lower semicontinuity of the relative entropy gives $S(\mathbb{Q} \mid \mathbb{P}) \leq \log \lambda_{0}^{-1}$. This implies that $\mathbb{Q} \ll \mathbb{P}$. Since $\mathbb{Q}, \mathbb{P} \in \mathcal{P}_{\phi}$, and $\mathbb{P}$ is ergodic, we have $\mathbb{Q}=\mathbb{P}$. The proof of $\mathcal{P}_{\text {eq }}(1)=\{\widehat{\mathbb{P}}\}$ is analogous. 


\subsection{Proof of Theorem 2.5}

We follow the strategy of [Fe3]. In what follows Assumption (C) is supposed to hold without further notice.

Lemma 4.1 For any $\boldsymbol{\omega}, \boldsymbol{\nu} \in \Omega_{\mathrm{fin}}$ there exists $\boldsymbol{\xi} \in \Omega_{\mathrm{fin}}$ such that $|\boldsymbol{\xi}| \leq \tau$ and

$$
\mathbb{P}(\boldsymbol{\omega} \boldsymbol{\nu})^{1-\alpha} \widehat{\mathbb{P}}(\boldsymbol{\omega} \boldsymbol{\nu})^{\alpha} \geq C_{\tau} \lambda_{0}[\mathbb{P}(\boldsymbol{\omega}) \mathbb{P}(\boldsymbol{\nu})]^{1-\alpha}[\widehat{\mathbb{P}}(\boldsymbol{\omega}) \widehat{\mathbb{P}}(\boldsymbol{\nu})]^{\alpha}
$$

for all $\alpha \in[0,1]$.

Proof. Let $\boldsymbol{\omega}, \boldsymbol{\nu} \in \Omega_{\mathrm{fin}}$. Assumption (C) gives that for some $\boldsymbol{\xi} \in \Omega_{t}$ with $t \leq \tau$,

$$
\frac{\mathbb{P}(\boldsymbol{\omega} \boldsymbol{\xi}) \widehat{\mathbb{P}}(\boldsymbol{\omega} \boldsymbol{\xi})}{[\mathbb{P}(\boldsymbol{\omega}) \mathbb{P}(\boldsymbol{\nu})]^{\alpha}[\widehat{\mathbb{P}}(\boldsymbol{\omega}) \widehat{\mathbb{P}}(\boldsymbol{\nu})]^{1-\alpha}} \geq C_{\tau}[\mathbb{P}(\boldsymbol{\omega}) \mathbb{P}(\boldsymbol{\nu})]^{1-\alpha}[\widehat{\mathbb{P}}(\boldsymbol{\omega}) \widehat{\mathbb{P}}(\boldsymbol{\nu})]^{\alpha}
$$

Lemma 3.4 gives

$$
\mathbb{P}^{\#}(\boldsymbol{\omega} \boldsymbol{\xi} \boldsymbol{\nu}) \leq \lambda_{0}^{-1} \mathbb{P}^{\#}(\boldsymbol{\omega} \boldsymbol{\xi}) \mathbb{P}^{\#}(\boldsymbol{\nu}) \leq \lambda_{0}^{-1} \sum_{\boldsymbol{\xi}^{\prime} \in \Omega_{t}} \mathbb{P}^{\#}\left(\boldsymbol{\omega} \boldsymbol{\xi}^{\prime}\right) \mathbb{P}^{\#}(\boldsymbol{\nu})=\lambda_{0}^{-1} \mathbb{P}^{\#}(\boldsymbol{\omega}) \mathbb{P}^{\#}(\boldsymbol{\nu})
$$

Hence, for $\alpha \in[0,1]$

$$
\mathbb{P}(\boldsymbol{\omega} \boldsymbol{\nu}) \widehat{\mathbb{P}}(\boldsymbol{\omega} \boldsymbol{\nu}) \leq \mathbb{P}(\boldsymbol{\omega} \boldsymbol{\nu})^{1-\alpha} \widehat{\mathbb{P}}(\boldsymbol{\omega} \boldsymbol{\nu})^{\alpha} \lambda_{0}^{-1}[\mathbb{P}(\boldsymbol{\omega}) \mathbb{P}(\boldsymbol{\nu})]^{\alpha}[\widehat{\mathbb{P}}(\boldsymbol{\omega}) \widehat{\mathbb{P}}(\boldsymbol{\nu})]^{1-\alpha},
$$

and the result follows.

Lemma 4.2 There exists a constant $C>0$ such that the following holds for all $\omega \in \Omega_{\mathrm{fin}}$, all $T \geq 1$ and all $\alpha \in[0,1]$ :

(1) $\sum_{\boldsymbol{\nu} \in \Omega_{T}} \mathbb{P}(\boldsymbol{\omega} \boldsymbol{\nu})^{1-\alpha} \widehat{\mathbb{P}}(\boldsymbol{\omega} \boldsymbol{\nu})^{\alpha} \geq C \mathrm{e}^{e_{T}(\alpha)} \mathbb{P}(\boldsymbol{\omega})^{1-\alpha} \widehat{\mathbb{P}}(\boldsymbol{\omega})^{\alpha}$.

(2) $\sum_{\boldsymbol{\nu} \in \Omega_{T}} \mathbb{P}(\boldsymbol{\nu} \boldsymbol{\omega})^{1-\alpha} \widehat{\mathbb{P}}(\boldsymbol{\nu} \boldsymbol{\omega})^{\alpha} \geq C \mathrm{e}^{e_{T}(\alpha)} \mathbb{P}(\boldsymbol{\omega})^{1-\alpha} \widehat{\mathbb{P}}(\boldsymbol{\omega})^{\alpha}$.

Proof. We prove only Part (1), the proof of Part (2) is similar. In the case $\tau=0$ the results follow immediately from Lemma 4.1 with $C=C_{0} \lambda_{0}$. Consider now the case $\tau \geq 1$. For any $\omega \in \Omega_{\mathrm{fin}}$ and any $\boldsymbol{\xi} \in \Omega_{t}$ with $t \leq \tau$, we deduce from Lemma 3.4

$$
\begin{aligned}
& \sum_{\nu \in \Omega_{T}} \mathbb{P}(\boldsymbol{\omega} \boldsymbol{\nu})^{1-\alpha} \widehat{\mathbb{P}}(\boldsymbol{\omega} \boldsymbol{\xi} \boldsymbol{\nu})^{\alpha} \leq \sum_{s=0}^{\tau} \sum_{\boldsymbol{\nu} \in \Omega_{T+s}} \mathbb{P}(\boldsymbol{\omega} \boldsymbol{\nu})^{1-\alpha} \widehat{\mathbb{P}}(\boldsymbol{\omega} \boldsymbol{\nu})^{\alpha} \\
& =\sum_{s=0}^{\tau} \sum_{\boldsymbol{\nu} \in \Omega_{T}, \boldsymbol{\eta} \in \Omega_{s}} \mathbb{P}(\boldsymbol{\omega} \boldsymbol{\nu} \boldsymbol{\eta})^{1-\alpha} \widehat{\mathbb{P}}(\boldsymbol{\omega} \boldsymbol{\nu} \boldsymbol{\eta})^{\alpha} \\
& \leq \lambda_{0}^{-1}\left(\sum_{s=0}^{\tau} \sum_{\boldsymbol{\eta} \in \Omega_{s}} \mathbb{P}(\boldsymbol{\eta})^{1-\alpha} \widehat{\mathbb{P}}(\boldsymbol{\eta})^{\alpha}\right)\left(\sum_{\boldsymbol{\nu} \in \Omega_{T}} \mathbb{P}(\boldsymbol{\omega} \boldsymbol{\nu})^{1-\alpha} \widehat{\mathbb{P}}(\boldsymbol{\omega} \boldsymbol{\nu})^{\alpha}\right) \\
& =\left(\lambda_{0}^{-1} \sum_{s=0}^{\tau} \mathrm{e}^{e_{s}(\alpha)}\right) \sum_{\boldsymbol{\nu} \in \Omega_{T}} \mathbb{P}(\boldsymbol{\omega} \boldsymbol{\nu})^{1-\alpha} \widehat{\mathbb{P}}(\boldsymbol{\omega} \boldsymbol{\nu})^{\alpha} \\
& \leq(\tau+1) \lambda_{0}^{-1} \sum_{\boldsymbol{\nu} \in \Omega_{T}} \mathbb{P}(\boldsymbol{\omega} \boldsymbol{\nu})^{1-\alpha} \widehat{\mathbb{P}}(\boldsymbol{\omega} \boldsymbol{\nu})^{\alpha}
\end{aligned}
$$


where, in the last line, we used the fact that $e_{s}(\alpha) \leq 0$ for $\alpha \in[0,1]$. Since the above estimate holds for all $\boldsymbol{\xi} \in \Omega_{\text {fin }}$ with $|\boldsymbol{\xi}| \leq \tau$, Lemma 4.1 allows us to conclude that

$$
\begin{aligned}
\sum_{\boldsymbol{\nu} \in \Omega_{T}} \mathbb{P}(\boldsymbol{\omega} \boldsymbol{\nu})^{1-\alpha} \widehat{\mathbb{P}}(\boldsymbol{\omega} \boldsymbol{\nu})^{\alpha} & \geq \frac{\lambda_{0}}{\tau+1} \sum_{\nu \in \Omega_{T}} C_{\tau} \lambda_{0} \mathbb{P}(\boldsymbol{\nu})^{1-\alpha} \widehat{\mathbb{P}}(\boldsymbol{\nu})^{\alpha} \mathbb{P}(\boldsymbol{\omega})^{1-\alpha} \widehat{\mathbb{P}}(\boldsymbol{\omega})^{\alpha} \\
& =\frac{C_{\tau} \lambda_{0}^{2}}{\tau+1} \mathrm{e}^{e_{T}(\alpha)} \mathbb{P}(\boldsymbol{\omega})^{1-\alpha} \widehat{\mathbb{P}}(\boldsymbol{\omega})^{\alpha}
\end{aligned}
$$

The super-additivity of the sequence $\left\{e_{T}(\alpha)\right\}_{T \geq 1}$ for $\alpha \in[0,1]$ is an immediate consequence of the last lemma. Invoking Fekete's lemma thus yields the following

Corollary 4.3 There exist a constant $c$ such that for all $\alpha \in[0,1]$ and all $T, T^{\prime} \geq 1$,

$$
e_{T+T^{\prime}}(\alpha) \geq e_{T}(\alpha)+e_{T^{\prime}}(\alpha)+c .
$$

In particular,

$$
\left.\left.e(\alpha)=\sup _{T \geq 1} \frac{1}{T}\left(e_{T}(\alpha)+c\right) \in\right]-\infty, 0\right]
$$

for all $\alpha \in[0,1]$.

Lemma 4.4 For any $\alpha \in] 0,1\left[\right.$ there exists $\overline{\mathbb{Q}} \in \mathcal{P}_{\phi}$ such that, for some $C>0$, all $T>0$ and all $\boldsymbol{\omega} \in \Omega_{T}$,

$$
C^{-1} \mathrm{e}^{-T e(\alpha)} \mathbb{P}(\boldsymbol{\omega})^{1-\alpha} \widehat{\mathbb{P}}(\boldsymbol{\omega})^{\alpha} \leq \overline{\mathbb{Q}}(\boldsymbol{\omega}) \leq C \mathrm{e}^{-T e(\alpha)} \mathbb{P}(\boldsymbol{\omega})^{1-\alpha} \widehat{\mathbb{P}}(\boldsymbol{\omega})^{\alpha} .
$$

Moreover, any such $\overline{\mathbb{Q}}$ is $\phi$-ergodic.

Proof. Throughout the proof, we fix $\alpha \in] 0,1[$ and let $C$ denote a strictly positive constant which does not depend on $T$, but whose value may differ from place to place.

Combining sub- and super-additivity, we can find $c>0$ such that

$$
e_{T}(\alpha)+e_{T^{\prime}}(\alpha)-c \leq e_{T+T^{\prime}}(\alpha) \leq e_{T}(\alpha)+e_{T^{\prime}}(\alpha)+c,
$$

for all $T, T^{\prime} \geq 1$. For each $T \geq 1$ let $\mathbb{Q}_{[T]} \in \mathcal{P}_{\phi^{T}}$ be defined as in the proof of Part (5) of Theorem 2.4, so that

$$
\mathbb{Q}_{[T]}(\boldsymbol{\omega})=\mathrm{e}^{-e_{T}(\alpha)} \mathbb{P}(\boldsymbol{\omega})^{1-\alpha} \widehat{\mathbb{P}}(\boldsymbol{\omega})^{\alpha}
$$

for all $\boldsymbol{\omega} \in \Omega_{T}$. Let $\mathbb{Q}$ be a limit point of the sequence $\left\{\mathbb{Q}_{[T]}\right\}_{T \geq 1}$, i.e., assume that for some sequence $T_{k} \uparrow \infty$ one has $\mathbb{Q}=\lim _{k \rightarrow \infty} \mathbb{Q}_{\left[T_{k}\right]}$. We claim that such a $\mathbb{Q}$ satisfies the estimate (4.63).

We first deal with the lower bound. Fix $T \geq 1$ and let $\boldsymbol{\omega} \in \Omega_{T}$. Then

$$
\mathbb{Q}(\boldsymbol{\omega})=\lim _{k \rightarrow \infty} \mathbb{Q}_{\left[T_{k}\right]}(\boldsymbol{\omega}),
$$

and Lemma 4.2 implies that for $T_{k}>T$,

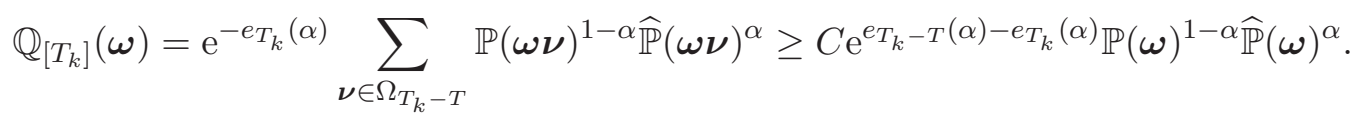

It follows from (4.64) that

$$
e_{T_{k}-T}(\alpha)-e_{T_{k}}(\alpha) \geq-e_{T}(\alpha)-c,
$$


while (4.62) allows us to write

$$
e(\alpha)=\sup _{t \geq 1} \frac{1}{t}\left(e_{t}(\alpha)+c\right) \geq \frac{1}{T}\left(e_{T}(\alpha)+c\right),
$$

and conclude that

$$
\mathbb{Q}_{\left[T_{k}\right]}(\boldsymbol{\omega}) \geq C \mathrm{e}^{-T e(\alpha)} \mathbb{P}(\boldsymbol{\omega})^{1-\alpha} \widehat{\mathbb{P}}(\boldsymbol{\omega})^{\alpha} .
$$

This inequality and (4.65) yield that the measure $\mathbb{Q}$ satisfies the lower bound in (4.63).

We now turn to the upper bound. For $T_{k}>T$ and $\omega \in \Omega_{T}$, Lemma 3.4 gives

$$
\mathbb{Q}_{\left[T_{k}\right]}(\boldsymbol{\omega})=\mathrm{e}^{-e_{T_{k}}(\alpha)} \sum_{\boldsymbol{\nu} \in \Omega_{T_{k}}-T} \mathbb{P}(\boldsymbol{\omega} \boldsymbol{\nu})^{1-\alpha} \widehat{\mathbb{P}}(\boldsymbol{\omega} \boldsymbol{\nu})^{\alpha} \leq C \mathrm{e}^{e_{T_{k}-T}(\alpha)-e_{T_{k}}(\alpha)} \mathbb{P}(\boldsymbol{\omega})^{1-\alpha} \widehat{\mathbb{P}}(\boldsymbol{\omega})^{\alpha} .
$$

It follows from (4.64) that

$$
e_{T_{k}-T}(\alpha)-e_{T_{k}}(\alpha) \leq-e_{T}(\alpha)+c
$$

and (4.52) yields

$$
e(\alpha)=\inf _{t \geq 1} \frac{1}{t}\left(e_{t}(\alpha)-c\right) \leq \frac{1}{T}\left(e_{T}(\alpha)-c\right)
$$

so that

$$
\mathbb{Q}_{\left[T_{k}\right]}(\boldsymbol{\omega}) \leq C \mathrm{e}^{-T e(\alpha)} \mathbb{P}(\boldsymbol{\omega})^{1-\alpha} \widehat{\mathbb{P}}(\boldsymbol{\omega})^{\alpha} .
$$

This inequality and (4.65) yield that the upper bound in (4.63) holds for the measure $\mathbb{Q}$.

Note that the measure $\mathbb{Q}$ needs not to be in $\mathcal{P}_{\phi}$. To deal with this point, we set

$$
\mathbb{Q}_{(T)}=\frac{1}{T} \sum_{t=0}^{T-1} \mathbb{Q} \circ \phi^{-t},
$$

and note that for $\boldsymbol{\omega} \in \Omega_{T^{\prime}}$

$$
\mathbb{Q} \circ \phi^{-t}(\boldsymbol{\omega})=\sum_{\boldsymbol{\nu} \in \Omega_{t}} \mathbb{Q}(\boldsymbol{\nu} \boldsymbol{\omega})
$$

The estimates (4.63) and Lemmas 3.4, 4.2 lead to

$$
C^{-1} \mathrm{e}^{e_{t}(\alpha)-\left(T^{\prime}+t\right) e(\alpha)} \mathbb{P}(\boldsymbol{\omega})^{1-\alpha} \widehat{\mathbb{P}}(\boldsymbol{\omega})^{\alpha} \leq \mathbb{Q} \circ \phi^{-t}(\boldsymbol{\omega}) \leq C \mathrm{e}^{e_{t}(\alpha)-\left(T^{\prime}+t\right) e(\alpha)} \mathbb{P}(\boldsymbol{\omega})^{1-\alpha} \widehat{\mathbb{P}}(\boldsymbol{\omega})^{\alpha} .
$$

Since (4.66) and (4.67) further imply $c \leq e_{t}(\alpha)-t e(\alpha) \leq-c$, we derive

$$
C^{-1} \mathrm{e}^{-T^{\prime} e(\alpha)} \mathbb{P}(\boldsymbol{\omega})^{1-\alpha} \widehat{\mathbb{P}}(\boldsymbol{\omega})^{\alpha} \leq \mathbb{Q} \circ \phi^{-t}(\boldsymbol{\omega}) \leq C \mathrm{e}^{-T^{\prime} e(\alpha)} \mathbb{P}(\boldsymbol{\omega})^{1-\alpha} \widehat{\mathbb{P}}(\boldsymbol{\omega})^{\alpha},
$$

for all $\boldsymbol{\omega} \in \Omega_{T^{\prime}}$. It follows that any limit point $\overline{\mathbb{Q}}$ of the sequence $\{\mathbb{Q}(T)\}_{T \geq 1}$, which belongs to $\mathcal{P}_{\phi}$ by construction, also satisfies the estimates (4.63).

Finally, we proceed to show that any $\overline{\mathbb{Q}} \in \mathcal{P}_{\phi}$ satisfying the estimates (4.63) is $\phi$-ergodic. To this end, let $\mathcal{C}_{1}$ and $\mathcal{C}_{2}$ be two cylinder subsets of $\Omega$, more explicitely

$$
\mathcal{C}_{i}=\left\{\omega \in \Omega \mid\left(\omega_{1}, \ldots, \omega_{r_{i}}\right) \in C_{i}\right\},
$$

where $r_{i}>0$ and $C_{i} \subset \Omega_{r_{i}}$. For $t \geq r_{1}$, the lower bound in (4.63) yields

$$
\begin{aligned}
\overline{\mathbb{Q}}\left(\mathcal{C}_{1} \cap \phi^{-t}\left(\mathcal{C}_{2}\right)\right) & =\sum_{\boldsymbol{\omega}_{i} \in C_{i}} \sum_{\boldsymbol{\xi} \in \Omega_{t-r_{1}}} \overline{\mathbb{Q}}\left(\boldsymbol{\omega}_{1} \boldsymbol{\xi} \boldsymbol{\omega}_{2}\right) \\
& \geq \sum_{\boldsymbol{\omega}_{i} \in C_{i}} \sum_{\boldsymbol{\xi} \in \Omega_{t-r_{1}}} C^{-1} \mathrm{e}^{-\left(t+r_{2}\right) e(\alpha)} \mathbb{P}\left(\boldsymbol{\omega}_{1} \boldsymbol{\xi} \boldsymbol{\omega}_{2}\right)^{1-\alpha} \widehat{\mathbb{P}}\left(\boldsymbol{\omega}_{1} \boldsymbol{\xi} \boldsymbol{\omega}_{2}\right)^{\alpha} .
\end{aligned}
$$


Invoking Lemma 4.1 and the fact that $e(\alpha) \leq 0$, we can write

$$
\begin{aligned}
\sum_{t=r_{1}}^{r_{1}+\tau} \overline{\mathbb{Q}}\left(\mathcal{C}_{1} \cap \phi^{-t}\left(\mathcal{C}_{2}\right)\right) & \geq \sum_{\boldsymbol{\omega}_{i} \in C_{i}} \sum_{t=0}^{\tau} \mathrm{e}^{-t e(\alpha)} \sum_{\boldsymbol{\xi} \in \Omega_{t}} C^{-1} \mathrm{e}^{-\left(r_{1}+r_{2}\right) e(\alpha)} \mathbb{P}\left(\boldsymbol{\omega}_{1} \boldsymbol{\xi} \boldsymbol{\omega}_{2}\right)^{1-\alpha} \widehat{\mathbb{P}}\left(\boldsymbol{\omega}_{1} \boldsymbol{\xi} \boldsymbol{\omega}_{2}\right)^{\alpha} \\
& \geq \sum_{\boldsymbol{\omega}_{i} \in C_{i}} C^{-1} C_{\tau} \lambda_{0}\left(\mathrm{e}^{-r_{1} e(\alpha)} \mathbb{P}\left(\boldsymbol{\omega}_{1}\right)^{1-\alpha} \widehat{\mathbb{P}}\left(\boldsymbol{\omega}_{1}\right)^{\alpha}\right)\left(\mathrm{e}^{-r_{2} e(\alpha)} \mathbb{P}\left(\boldsymbol{\omega}_{2}\right)^{1-\alpha} \widehat{\mathbb{P}}\left(\boldsymbol{\omega}_{2}\right)^{\alpha}\right)
\end{aligned}
$$

The upper bound in (4.63) further gives

$$
\begin{aligned}
(1+\tau) \sup _{t \geq 0} \overline{\mathbb{Q}}\left(\mathcal{C}_{1} \cap \phi^{-t}\left(\mathcal{C}_{2}\right)\right) & \geq \sum_{t=r_{1}}^{r_{1}+\tau} \overline{\mathbb{Q}}\left(\mathcal{C}_{1} \cap \phi^{-t}\left(\mathcal{C}_{2}\right)\right) \\
& \geq \sum_{\boldsymbol{\omega}_{i} \in C_{i}} C^{-3} C_{\tau} \lambda_{0} \overline{\mathbb{Q}}\left(\boldsymbol{\omega}_{1}\right) \overline{\mathbb{Q}}\left(\boldsymbol{\omega}_{2}\right)=C^{-3} C_{\tau} \lambda_{0} \overline{\mathbb{Q}}\left(\mathcal{C}_{1}\right) \overline{\mathbb{Q}}\left(\mathcal{C}_{2}\right)
\end{aligned}
$$

from which we conclude that the lower bound

$$
\sup _{t \geq 0} \overline{\mathbb{Q}}\left(\mathcal{C}_{1} \cap \phi^{-t}\left(\mathcal{C}_{2}\right)\right) \geq C \overline{\mathbb{Q}}\left(\mathcal{C}_{1}\right) \overline{\mathbb{Q}}\left(\mathcal{C}_{2}\right)
$$

holds for any cylinder subsets $\mathcal{C}_{1}, \mathcal{C}_{2} \subset \Omega$. Since cylinder sets generate the Borel $\sigma$-field $\mathcal{F}$, given $\mathcal{B}_{1}, \mathcal{B}_{2} \in \mathcal{F}$ and $\epsilon>0$ there are two cylinders sets $\mathcal{C}_{1}$ and $\mathcal{C}_{2}$ such that $\overline{\mathbb{Q}}\left(\mathcal{B}_{i} \Delta \mathcal{C}_{i}\right)<\epsilon$. It follows that $\overline{\mathbb{Q}}\left(\phi^{-t}\left(\mathcal{B}_{2}\right) \Delta \phi^{-t}\left(\mathcal{C}_{2}\right)\right)=\overline{\mathbb{Q}}\left(\phi^{-t}\left(\mathcal{B}_{2} \Delta \mathcal{C}_{2}\right)\right)<\epsilon$ and hence

$$
\left|\overline{\mathbb{Q}}\left(\mathcal{B}_{1} \cap \phi^{-t}\left(\mathcal{B}_{2}\right)\right)-\overline{\mathbb{Q}}\left(\mathcal{C}_{1} \cap \phi^{-t}\left(\mathcal{C}_{2}\right)\right)\right|<2 \epsilon,
$$

for any $t \geq 0$. Accordingly,

$$
\sup _{t \geq 0} \overline{\mathbb{Q}}\left(\mathcal{B}_{1} \cap \phi^{-t}\left(\mathcal{B}_{2}\right)\right) \geq C \overline{\mathbb{Q}}\left(\mathcal{C}_{1}\right) \overline{\mathbb{Q}}\left(\mathcal{C}_{2}\right)-2 \epsilon \geq C\left(\overline{\mathbb{Q}}\left(\mathcal{B}_{1}\right)-\epsilon\right)\left(\overline{\mathbb{Q}}\left(\mathcal{B}_{2}\right)-\epsilon\right)-2 \epsilon
$$

and by an appropriate choice of $\epsilon>0$ we can achieve

$$
\sup _{t \geq 0} \overline{\mathbb{Q}}\left(\mathcal{B}_{1} \cap \phi^{-t}\left(\mathcal{B}_{2}\right)\right) \geq \frac{C}{2} \overline{\mathbb{Q}}\left(\mathcal{B}_{1}\right) \overline{\mathbb{Q}}\left(\mathcal{B}_{2}\right) .
$$

It follows that if $\overline{\mathbb{Q}}\left(\mathcal{B}_{1}\right)>0$ and $\overline{\mathbb{Q}}\left(\mathcal{B}_{2}\right)>0$, then there exists $t$ such that $\overline{\mathbb{Q}}\left(\mathcal{B}_{1} \cap \phi^{-t}\left(\mathcal{B}_{2}\right)\right)>0$ and Theorem 1.5 in [Wa] implies that $\overline{\mathbb{Q}}$ is $\phi$-ergodic.

Remark. Since all limit points $\overline{\mathbb{Q}}$ of the sequence $\{\mathbb{Q}(T)\}_{T \geq 1}$ satisfy the estimate (4.63), a standard relative entropy argument yields that they are all mutually absolutely continuous. Hence, if one of them is ergodic, they all coincide and $\overline{\mathbb{Q}}=\lim _{T \rightarrow \infty} \mathbb{Q}_{(T)}$. We will not make use of this observation in the sequel.

We are now ready to prove Theorem 2.5. The case $\alpha \in\{0,1\}$ follows from Part (8) of Theorem 2.4. Thus, we need only to consider $\alpha \in] 0,1[$. We start by noticing that if $\overline{\mathbb{Q}}$ is as in Lemma 4.4, then the 
estimates (4.63) imply

$$
\begin{aligned}
\varsigma\left(\overline{\mathbb{Q}}^{(\alpha)}\right) & =-\lim _{T \rightarrow \infty} \frac{1}{T} \sum_{\boldsymbol{\omega} \in \Omega_{T}} \overline{\mathbb{Q}}(\boldsymbol{\omega}) \log \mathbb{P}(\boldsymbol{\omega})^{1-\alpha} \widehat{\mathbb{P}}(\boldsymbol{\omega})^{\alpha} \\
& \lesseqgtr-\lim _{T \rightarrow \infty} \frac{1}{T} \sum_{\boldsymbol{\omega} \in \Omega_{T}} \overline{\mathbb{Q}}(\boldsymbol{\omega}) \log \left(C^{ \pm 1} \mathrm{e}^{T e(\alpha)} \overline{\mathbb{Q}}(\omega)\right) \\
& =-e(\alpha)-\lim _{T \rightarrow \infty} \frac{1}{T} \sum_{\boldsymbol{\omega} \in \Omega_{T}} \overline{\mathbb{Q}}(\boldsymbol{\omega}) \log \overline{\mathbb{Q}}(\omega) \\
& =-e(\alpha)+h_{\phi}(\overline{\mathbb{Q}}),
\end{aligned}
$$

and hence $\overline{\mathbb{Q}} \in \mathcal{P}_{\text {eq }}(\alpha)$. To show that $\overline{\mathbb{Q}}$ is the only element of $\mathcal{P}_{\text {eq }}(\alpha)$, let $\mathbb{Q} \in \mathcal{P}_{\text {eq }}(\alpha)$. The upper bound in (4.63) yields

$$
S\left(\mathbb{Q}_{T} \mid \overline{\mathbb{Q}}_{T}\right)=\sum_{\boldsymbol{\omega} \in \Omega_{T}} \mathbb{Q}(\boldsymbol{\omega}) \log \frac{\mathbb{Q}(\boldsymbol{\omega})}{\overline{\mathbb{Q}}(\boldsymbol{\omega})} \leq-S\left(\mathbb{Q}_{T}\right)+\log C+T e(\alpha)+T \varsigma\left(\mathbb{Q}^{(\alpha)}\right),
$$

and hence

$$
0 \leq \limsup _{T \rightarrow \infty} \frac{1}{T} S\left(\mathbb{Q}_{T} \mid \overline{\mathbb{Q}}_{T}\right) \leq-h_{\phi}(\mathbb{Q})+e(\alpha)+\varsigma\left(\mathbb{Q}^{(\alpha)}\right)=e(\alpha)-f\left(\mathbb{Q}^{(\alpha)}\right)=0 .
$$

Combining Lemmata 3.4 and 4.4, we derive

$$
\overline{\mathbb{Q}}_{T+T^{\prime}}\left(\boldsymbol{\omega} \boldsymbol{\omega}^{\prime}\right) \leq C^{3} \lambda_{0}^{-2} \overline{\mathbb{Q}}_{T}(\boldsymbol{\omega}) \overline{\mathbb{Q}}_{T^{\prime}}\left(\boldsymbol{\omega}^{\prime}\right),
$$

for all $T, T^{\prime} \geq 1, \boldsymbol{\omega} \in \Omega_{T}$ and $\boldsymbol{\omega}^{\prime} \in \Omega_{T^{\prime}}$. Writing

$$
a_{T}=S\left(\mathbb{Q}_{T} \mid \overline{\mathbb{Q}}_{T}\right)=-S\left(\mathbb{Q}_{T}\right)-\mathbb{Q}\left[\log \overline{\mathbb{Q}}_{T}\right]
$$

we deduce from (4.69) and the subadditivity of entropy that

$$
a_{T+T^{\prime}} \geq a_{T}+a_{T^{\prime}}-K
$$

where $K=\log \left(C^{3} \lambda_{0}^{-2}\right)>0$. Fekete's lemma and (4.68) then give

$$
0=\lim _{T \rightarrow \infty} \frac{1}{T} a_{T}=\sup _{T \geq 1} \frac{1}{T}\left(a_{T}-K\right)
$$

from which we conclude that $S\left(\mathbb{Q}_{T} \mid \overline{\mathbb{Q}}_{T}\right) \leq K$ for all $T \geq 1$. By the lower semicontinuity of the relative entropy we get

$$
S(\mathbb{Q} \mid \overline{\mathbb{Q}}) \leq \liminf _{T \rightarrow \infty} S\left(\mathbb{Q}_{T} \mid \overline{\mathbb{Q}}_{T}\right) \leq K,
$$

and so $\mathbb{Q} \ll \overline{\mathbb{Q}}$. Since $\mathbb{Q}, \overline{\mathbb{Q}} \in \mathcal{P}_{\phi}$ and $\overline{\mathbb{Q}}$ is $\phi$-ergodic, we have $\mathbb{Q}=\overline{\mathbb{Q}}$. Hence, $\mathcal{P}_{\text {eq }}(\alpha)$ is a singleton for all $\alpha \in] 0,1[$, and the differentiability of $e(\alpha)$ follows from Part (6) of Theorem 2.4. The proof of Theorem 2.5 is complete. 


\subsection{Proof of Proposition 2.6}

We argue by contradiction. If the statement is not true then, for each $t \geq 0$, there exist $\boldsymbol{\omega}_{t}, \boldsymbol{\nu}_{t} \in \Omega_{\mathrm{fin}}$ such that

$$
\max _{\substack{\boldsymbol{\xi} \in \Omega_{\mathrm{fin}} \\|\boldsymbol{\xi}| \leq t}} \frac{\mathbb{P}\left(\boldsymbol{\omega}_{t} \boldsymbol{\xi} \boldsymbol{\nu}_{t}\right) \widehat{\mathbb{P}}\left(\boldsymbol{\omega}_{t} \boldsymbol{\xi} \boldsymbol{\nu}_{t}\right)}{\mathbb{P}\left(\boldsymbol{\omega}_{t}\right) \mathbb{P}\left(\boldsymbol{\nu}_{t}\right) \widehat{\mathbb{P}}\left(\boldsymbol{\omega}_{t}\right) \widehat{\mathbb{P}}\left(\boldsymbol{\nu}_{t}\right)} \leq \frac{1}{1+t}
$$

and hence

$$
\lim _{t \rightarrow \infty} \frac{\mathbb{P}\left(\boldsymbol{\omega}_{t} \boldsymbol{\xi} \boldsymbol{\nu}_{t}\right) \widehat{\mathbb{P}}\left(\boldsymbol{\omega}_{t} \boldsymbol{\xi} \boldsymbol{\nu}_{t}\right)}{\mathbb{P}\left(\boldsymbol{\omega}_{t}\right) \mathbb{P}\left(\boldsymbol{\nu}_{t}\right) \widehat{\mathbb{P}}\left(\boldsymbol{\omega}_{t}\right) \widehat{\mathbb{P}}\left(\boldsymbol{\nu}_{t}\right)}=0
$$

for all $\xi \in \Omega_{\text {fin }}$. In terms of the OR process $(\widehat{\mathcal{J}}, \widehat{\rho})$, this can be rewritten as

$$
\lim _{t \rightarrow \infty} \frac{\operatorname{tr}\left(\left(\Phi_{\boldsymbol{\omega}_{t}}^{*} \otimes \widehat{\Phi}_{\boldsymbol{\omega}_{t}}^{*}\right)[\rho \otimes \widehat{\rho}]\left(\Phi_{\boldsymbol{\xi}} \otimes \widehat{\Phi}_{\boldsymbol{\xi}}\right)\left[\left(\Phi_{\boldsymbol{\nu}_{t}} \otimes \widehat{\Phi}_{\boldsymbol{\nu}_{t}}\right)[\mathbb{1} \otimes \mathbb{1}]\right]\right)}{\operatorname{tr}\left(\left(\Phi_{\boldsymbol{\omega}_{t}}^{*} \otimes \widehat{\Phi}_{\boldsymbol{\omega}_{t}}^{*}\right)[\rho \otimes \widehat{\rho}]\right) \operatorname{tr}\left((\rho \otimes \widehat{\rho})\left(\Phi_{\boldsymbol{\nu}_{t}} \otimes \widehat{\Phi}_{\boldsymbol{\nu}_{t}}\right)[\mathbb{1} \otimes \mathbb{1}]\right)}=0,
$$

where $\Phi_{\boldsymbol{\omega}}=\Phi_{\omega_{1}} \circ \cdots \circ \Phi_{\omega_{T}}$ for $\boldsymbol{\omega}=\left(\omega_{1}, \ldots, \omega_{T}\right)$. Since

$$
\operatorname{tr}\left((\rho \otimes \widehat{\rho})\left(\Phi_{\boldsymbol{\nu}_{t}} \otimes \widehat{\Phi}_{\boldsymbol{\nu}_{t}}\right)[\mathbb{1} \otimes \mathbb{1}]\right) \leq \operatorname{tr}\left(\left(\Phi_{\boldsymbol{\nu}_{t}} \otimes \widehat{\Phi}_{\boldsymbol{\nu}_{t}}\right)[\mathbb{1} \otimes \mathbb{1}]\right),
$$

we also have

$$
\lim _{t \rightarrow \infty} \frac{\operatorname{tr}\left(\left(\Phi_{\boldsymbol{\omega}_{t}}^{*} \otimes \widehat{\Phi}_{\boldsymbol{\omega}_{t}}^{*}\right)[\rho \otimes \widehat{\rho}]\left(\Phi_{\boldsymbol{\xi}} \otimes \widehat{\Phi}_{\boldsymbol{\xi}}\right)\left[\left(\Phi_{\boldsymbol{\nu}_{t}} \otimes \widehat{\Phi}_{\boldsymbol{\nu}_{t}}\right)[\mathbb{1} \otimes \mathbb{1}]\right]\right)}{\operatorname{tr}\left(\left(\Phi_{\boldsymbol{\omega}_{t}}^{*} \otimes \widehat{\Phi}_{\boldsymbol{\omega}_{t}}^{*}\right)[\rho \otimes \widehat{\rho}]\right) \operatorname{tr}\left(\left(\Phi_{\boldsymbol{\nu}_{t}} \otimes \widehat{\Phi}_{\boldsymbol{\nu}_{t}}\right)[\mathbb{1} \otimes \mathbb{1}]\right)}=0 .
$$

By passing to a subsequence $t_{n} \rightarrow \infty$, we can assume that the limits

$$
\varrho=\lim _{n \rightarrow \infty} \frac{\left(\Phi_{\boldsymbol{\omega}_{t_{n}}}^{*} \otimes \widehat{\Phi}_{\boldsymbol{\omega}_{t_{n}}}^{*}\right)[\rho \otimes \widehat{\rho}]}{\operatorname{tr}\left(\left(\Phi_{\boldsymbol{\omega}_{t_{n}}}^{*} \otimes \widehat{\Phi}_{\boldsymbol{\omega}_{t_{n}}}^{*}\right)[\rho \otimes \widehat{\rho}]\right)}, \quad \varrho^{\prime}=\lim _{n \rightarrow \infty} \frac{\left(\Phi_{\boldsymbol{\nu}_{t_{n}}} \otimes \widehat{\Phi}_{\boldsymbol{\nu}_{t_{n}}}\right)[\mathbb{1} \otimes \mathbb{1}]}{\operatorname{tr}\left(\left(\Phi_{\boldsymbol{\nu}_{t_{n}}} \otimes \widehat{\Phi}_{\boldsymbol{\nu}_{t_{n}}}\right)[\mathbb{1} \otimes \mathbb{1}]\right)}
$$

exist and define density matrices on $\mathcal{H} \otimes \mathcal{H}$ such that

$$
\operatorname{tr}\left(\varrho\left(\Phi_{\boldsymbol{\xi}} \otimes \widehat{\Phi}_{\boldsymbol{\xi}}\right)\left[\varrho^{\prime}\right]\right)=0
$$

for all $\xi \in \Omega_{\mathrm{fin}}$. Hence, the positive map $\Psi=\sum_{a \in \mathcal{A}} \Phi_{a} \otimes \widehat{\Phi}_{a}$ satisfies

$$
\operatorname{tr}\left(\varrho \Psi^{t}\left[\varrho^{\prime}\right]\right)=\sum_{\boldsymbol{\xi} \in \Omega_{t}} \operatorname{tr}\left(\varrho\left(\Phi_{\boldsymbol{\xi}} \otimes \widehat{\Phi}_{\boldsymbol{\xi}}\right)\left[\varrho^{\prime}\right]\right)=0
$$

for all $t \geq 0$, which contradicts the assumption that $\Psi$ is irreducible; see [EHK, Lemma 2.1].

\subsection{Proof of Theorem 2.8}

Lemma 3.4 and Assumption (D) give that there exist constants $c$ and $c(\alpha)$ such that for all $T, T^{\prime} \geq 1$, $\alpha \in \mathbb{R}$ and $\mathbb{Q} \in \mathcal{P}_{\phi}$

$$
\begin{aligned}
& e_{T}(\alpha)+e_{T^{\prime}}(\alpha)-c(\alpha) \leq e_{T+T^{\prime}}(\alpha) \leq e_{T}(\alpha)+e_{T^{\prime}}(\alpha)+c(\alpha) \\
& \mathbb{Q}\left[-\log \mathbb{P}_{T}\right]+\mathbb{Q}\left[-\log \mathbb{P}_{T^{\prime}}\right]-c \leq \mathbb{Q}\left[-\log \mathbb{P}_{T+T^{\prime}}\right] \leq \mathbb{Q}\left[-\log \mathbb{P}_{T}\right]+\mathbb{Q}\left[-\log \mathbb{P}_{T^{\prime}}\right]+c
\end{aligned}
$$


By Fekete's lemma the limits $e(\alpha)=\lim _{T \rightarrow \infty} \frac{1}{T} e_{T}(\alpha)$ and $\varsigma(\mathbb{Q})=\lim _{T \rightarrow \infty} \frac{1}{T} \mathbb{Q}\left[-\log \mathbb{P}_{T}\right]$ exist and are finite. In particular, the relations

$$
\inf _{T \geq 1} \frac{1}{T}\left(\mathbb{Q}\left[-\log \mathbb{P}_{T}\right]-c\right)=\varsigma(\mathbb{Q})=\sup _{T \geq 1} \frac{1}{T}\left(\mathbb{Q}\left[-\log \mathbb{P}_{T}\right]+c\right),
$$

imply that the map $\mathcal{P}_{\phi} \ni \mathbb{Q} \mapsto \varsigma(\mathbb{Q})$ is continuous. Going back to the proof of Theorem 2.4 (5), we can apply Lemma 2.3 in $[\mathrm{CFH}]$ to the sequence $f_{T}=1 /\left(D_{0} \mathbb{P}_{T}^{\#}\right)$ to complement (4.57) with the estimate

$$
\liminf _{k \rightarrow \infty} Q_{T_{k}}\left[\frac{1}{T_{k}} \log \mathbb{P}_{T_{k}}^{\#}\right] \geq \lim _{T \rightarrow \infty} \mathbb{Q}\left[\frac{1}{T} \log \mathbb{P}_{T}^{\#}\right]
$$

which, together with (4.57), implies

$$
\lim _{k \rightarrow \infty}(1-\alpha) Q_{T_{k}}\left[\frac{1}{T_{k}} \log \mathbb{P}_{T_{k}}\right]+\alpha Q_{T_{k}}\left[\frac{1}{T_{k}} \log \widehat{\mathbb{P}}_{T_{k}}\right]=-\varsigma\left(\mathbb{Q}^{(\alpha)}\right) .
$$

With this addition, the proof of Parts (5-6) of Theorem 2.4 now extend to all $\alpha \in \mathbb{R}$. Finally, to prove that $\mathcal{P}_{\text {eq }}(\alpha)$ is a singleton for all $\alpha \in \mathbb{R}$, one follows the arguments of Section 4.2. Replacing Assumption (C) with Assumption (D) one easily shows that Lemmata 4.2 and 4.4 also extend to all

$\alpha \in \mathbb{R}$. The details are considerably simpler than in Section 4.2 and we leave them to the interested reader.

\subsection{Proof of Proposition 2.9}

We argue by contradiction: if the statement is not true, we can find two sequences $\boldsymbol{\omega}_{n}$ and $\nu_{n}$ in $\Omega_{\text {fin }}$ such that

$$
\lim _{n \rightarrow \infty} \frac{\mathbb{P}\left(\boldsymbol{\omega}_{n} \boldsymbol{\nu}_{n}\right)}{\mathbb{P}\left(\boldsymbol{\omega}_{n}\right) \mathbb{P}\left(\boldsymbol{\nu}_{n}\right)}=0 .
$$

Passing to subsequences one easily deduces from (4.70) that there exist two density matrices $\varrho$ and $\varrho^{\prime}$ on $\mathcal{H}$ and some $a \in \mathcal{A}$ such that $\boldsymbol{\nu}_{n}=a \tilde{\boldsymbol{\nu}}_{n}$ and

$$
\varrho=\lim _{n \rightarrow \infty} \frac{\Phi_{\boldsymbol{\omega}_{n}}^{*}(\rho)}{\operatorname{tr}\left(\Phi_{\boldsymbol{\omega}_{n}}^{*}(\rho)\right)}, \quad \varrho^{\prime}=\lim _{n \rightarrow \infty} \frac{\Phi_{\tilde{\boldsymbol{\nu}}_{n}}(\mathbb{1})}{\operatorname{tr}\left(\Phi_{\tilde{\boldsymbol{\nu}}_{n}}(\mathbb{1})\right)} .
$$

It follows that $\operatorname{tr}\left(\varrho \Phi_{a}\left(\varrho^{\prime}\right)\right)=0$ which contradicts the assumption that $\Phi_{a}$ is positivity improving.

\subsection{Proof of Theorem 2.12}

The observation

$$
c_{T}=\frac{1}{2} \sum_{\omega \in \Omega_{T}} \min \left(\mathbb{P}_{T}(\omega), \widehat{\mathbb{P}}_{T}(\omega)\right) \leq \frac{1}{2} \sum_{\omega \in \Omega_{T}} \mathbb{P}_{T}(\omega)^{1 / 2} \widehat{\mathbb{P}}_{T}(\omega)^{1 / 2},
$$

yields $\log c_{T} \leq e_{T}\left(\frac{1}{2}\right)-\log 2$, and Theorem 2.4 (1) gives Part (1). To prove Part (2), note that Eq. (2.42) implies

$$
c_{T} \geq \frac{1}{2} \mathbb{P}_{T}\left(\underline{\mathcal{T}}_{T}^{c}\right)=\frac{1}{2} \mathbb{P}_{T}\left(\left\{\omega \in \Omega_{T} \mid \sigma_{T}(\omega)<0\right\}\right) .
$$


Since the function $e(\alpha)$ has a global minimum at $\alpha=\frac{1}{2}$, one has $0 \in \partial e\left(\frac{1}{2}\right)$, and Theorem 2.10 (2) gives

$$
\liminf _{T \rightarrow \infty} \frac{1}{T} \log c_{T} \geq-I\left(-\partial^{+} e\left(\frac{1}{2}\right)\right) .
$$

Part (2) thus follows from Eq. (2.37). Finally, under Assumption (C), Theorem 2.5 (2) and the above argument yield $e^{\prime}\left(\frac{1}{2}\right)=0$, and so Part (3) follows from Parts (1) and (2).

\section{References}

[AAV] Aharonov, Y., Albert, D.Z., and Vaidman, L.: How the result of a measurement of a component of the spin of a spin-1/2 particle can turn out to be 100. Phys. Rev. Lett. 60, 1351 (1988).

[AD] Acz̀el, J., and Daròczy, Z.: On Measures of Information and their Characterizations. Academic Press, New York, 1975.

[ABL] Aharonov, Y., Bergmann, P.G., and Lebowitz, J.L.: Time symmetry in the quantum process of measurement. Phys. Rev. 134, B1410-B1416 (1964).

[AV] Aharonov, Y., and Vaidman, L.: The two-state vector formalism of quantum mechanics: an updated review. In: Time in Quantum Mechanics, Volume 1. G. Muga, R. Sala Mayato, I. Egusquiza (eds.). Lecture Notes in Physics 734, 399-447, 2nd ed. Springer, Berlin, 2008.

[Ba] Baladi, V.: Positive Transfer Operators and Decay of Correlations. Advanced Series in Nonlinear Dynamics 16. World Scientific, River Edge, NJ, 2000.

[BaL] Barreira, L.: Almost additive thermodynamic formalism: some recent developments. Rev. Math. Phys. 22, 1147-1179 (2010).

[BaY] Barsheshat, Y.: Masters thesis, McGill, 2015.

[BSS] Batalhão, T.B., Souza, A.M., Sarthour, R.S., Oliveira, I.S., Paternostro, M., Lutz, E., and Serra, R.M.: Irreversibility and the arrow of time in a quenched quantum system. Phys. Rev. Lett. 115, 190601 (2015).

[BB] Bauer, M., and Bernard, D.: Convergence of repeated quantum nondemolition measurements and wave-function collapse. Phys. Rev. A 84, 044103 (2011).

[BBB] Bauer, M., Benoist, T., and Bernard, D.: Repeated quantum non-demolition measurements: convergence and continuous-time limit. Ann. Henri Poincaré 14, 639-679 (2013).

[BFFS] Ballesteros, M., Fraas, M., Fröhlich, J., and Schubnel, B.: Indirect acquisition of information in quantum mechanics. J. Stat. Phys. 162, 924-958 (2016).

[BFS] Blanchard, P., Fröhlich, J., and Schubnel, B.: A "Garden of Forking Paths" - the quantum mechanics of histories of events. Preprint, arXiv 1603.09664 [quant-ph], (2016).

[BG] Barchielli A., and Gregoratti, M.: Quantum Trajectories and Measurements in Continuous Time: The Diffusive Case. Lecture Notes in Physics 782. Springer, Berlin, 2009. 
On entropy production of repeated quantum measurements I

[BJPP1] Benoist, T., Jakšić, V., Pautrat, Y., and Pillet, C.-A.: On entropy production of repeated quantum measurements II. Examples. In preparation.

[BJPP2] Benoist, T., Jakšić, V., Pautrat, Y., and Pillet, C.-A.: On the nature of the quantum detailed balance condition. In preparation.

[BJPP3] Benoist, T., Jakšić, V., Pautrat, Y., and Pillet, C.-A.: On the Rényi entropy of repeated quantum measurements. In preparation.

[Bo] Bohm, D.: Quantum Theory. Prentice Hall, New York, 1951.

[BV] Bomfim, T., and Varandas, P.: Multifractal analysis of the irregular set for almost-additive sequences via large deviations. Nonlinearity 28, 3563-3585 (2015).

[Bo1] Bowen, R.: Some systems with unique equilibrium state. Math. Systems Theory 8, 193202 (1974).

[Bo2] Bowen, R.: Equilibrium States and the Ergodic Theory of Anosov Diffeomorphisms. Lecture Notes in Mathematics 470. Springer, Berlin, 1975.

[Ca] Carmichael, H.: An Open Systems Approach to Quantum Optics. Lecture Notes in Physics Monographs M 18. Springer, Berlin, 1993.

[CHT] Campisi, M., Hänggi P., and Talkner P.: Colloquium: Quantum fluctuation relations: Foundations and applications. Rev. Mod. Phys. 83, 771-791 (2011).

[CFH] Cao, Y.-L., Feng, D.-J., and Huang, W.: The thermodynamic formalism for sub-additive potentials. Discrete Contin. Dyn. Syst. 20, 639-657 (2008).

[Cr1] Crooks, G.E.: Entropy production fluctuation theorem and the nonequilibrium work relation for free energy differences. Phys. Rev. E 60, 2721 (1999).

[Cr2] Crooks, G.E.: Quantum operation time reversal. Phys. Rev. A 77, 034101 (2008).

[CZC] Chen, Y., Zhao, Y., and Cheng, W.-C.: Sub-additive pressure on a Borel set. Acta Math. Scient. 35, 1203-1213 (2015).

[Da] Davies, E.B.: Quantum Theory of Open Systems. Academic Press, London, 1976.

[dH] den Hollander, F.: Large Deviations. Fields Institute Monographs. AMS, Providence, 2000.

[Der] Derriennic, Y.: Un théorème ergodique presque sous-additif. Ann. Proba. 11, 669-677 (1983).

[DL] Deffner, S., and Lutz, E.: Nonequilibrium entropy production for open quantum systems. Phys. Rev. Lett. 107, 140404 (2011).

[DZ] Dembo, A., and Zeitouni, O.: Large Deviations Techniques and Applications. Second edition. Applications of Mathematics 38. Springer, New York, 1998.

[Do] Dobrushin, R.L.: A Gibbsian representation for non-Gibbsian fields. Lecture given at the workshop "Probability and Physics", September 1995, Renkum, Netherlands. 
[DoS] Dobrushin, R.L., and Shlosman S.B.: "Non-Gibbsian" states and their Gibbs description. Comm. Math. Phys. 200, 125-179 (1999).

[ECM] Evans, D.J., Cohen, E.G.D., and Morriss, G.P.: Probability of second law violation in shearing steady flows. Phys. Rev. Lett. 71, 2401-2404 (1993).

[Ed] Eddington, A.S.: The Nature of the Physical World. McMillan, London, 1928.

[EHK] Evans, D.E., and Høegh-Krohn, R.: Spectral properties of positive maps on $C^{*}$-algebras. J. London. Math. Soc. 17, 345-355 (1978).

[EHM] Esposito, M., Harbola, U., and Mukamel, S.: Nonequilibrium fluctuations, fluctuation theorems, and counting statistics in quantum systems. Rev. Mod. Phys. 81, 1665-1702 (2009).

[El] Ellis, R.S.: Entropy, Large Deviations, and Statistical Mechanics. Springer, Berlin, 1985. Reprinted in the series Classics of Mathematics (2006).

[ES] Evans, D.J., and Searles, D.J.: Equilibrium microstates which generate second law violating steady states. Phys Rev. E 50, 1645-1648 (1994).

[Fa] Falconer, K.J.: Sub-self-similar set. Transactions AMS 347, 3121-3129 (1995).

[FNW] Fannes, M., Nachtergaele, B., and Werner, R.F.: Finitely correlated states on quantum spin chains. Comm. Math. Phys. 144, 443-490 (1992).

[Fe1] Feng, D.-J.: The variational principle for products of non-negative matrices. Nonlinearity 17, 447-457 (2004).

[Fe2] Feng D.-J.: Lyapunov exponents for products of matrices and multifractal analysis. Part I: Positive matrices. Israel J. Math. 138, 353-376 (2003).

[Fe3] Feng, D.-J.: Lyapounov exponents for products of matrices and multifractal analysis. Part II: General matrices. Israel J. Math. 170, 355-394 (2009).

[FL] Feng, D.-J., and Lau K.-S.: The pressure function for products of non-negative matrices. Math. Res. Lett. 9, 363-378 (2002).

[FK] Feng, D.-J., and Känemäki, A.: Equilibrium states for the pressure function for products of matrices. Disc. Cont. Dyn. Syst. 30, 699-708 (2011).

[Fe] Fernandez, R.: Gibbsianness and non-Gibbsianness in lattice random fields. In Mathematical Statistical Physics. A. Bovier, J. Dalibard, F. Dunlop, A. van Enter, and F. den Hollander (eds.). Elsevier, 2006.

[FS] Falconer, K.J., and Sloan, A.: Continuity of subadditive pressure for self-affine sets. Real Analysis Exchange 34, 1-16, (2008/2009).

[GC1] Gallavotti, G., and Cohen, E.G.D.: Dynamical ensembles in nonequilibrium statistical mechanics. Phys. Rev. Lett. 74, 2694-2697 (1995).

[GC2] Gallavotti, G., and Cohen, E.G.D.: Dynamical ensembles in stationary states. J. Stat. Phys. 80, 931-970 (1995). 
On entropy production of repeated quantum measurements I

[GPP] Grigolini, P., Pala, G.M., and Palatella, L.: Quantum measurement and entropy production. Phys. Lett. A 285, 49-54 (2001).

[He] Heisenberg, W.: Über den anschaulichen Inhalt der quantentheoretischen Kinematik und Mechanik. Z. Phys. 43, 172-198 (1927).

[Ho] Holevo, A.S.: Statistical Structure of Quantum Theory. Lecture Notes in Physics Monographs M 67, Springer, Berlin, 2001.

[HMPZ] Halliwel, J.J., Pérez-Mercader, J., and Zurek, W.H. (eds.): Physical Origins of Time Asymmetry. Cambridge University Press, Cambridge, 1996.

[IY] Iommi, G., Yayama, Y.: Almost-additive thermodynamic formalism for countable Markov shifts. Nonlinearity 25, 165-191 (2012).

[Jak] Jakšić, V.: Lectures on Entropy. Preprint, McGill, 2015.

[JNPPS] Jakšić, V., Nersesyan, V., Pillet, C.-A., Porta, M., and Shirikyan, A.: In preparation.

[JOPP] Jakšić, V., Ogata, Y., Pautrat, Y., and Pillet, C.-A.: Entropic fluctuations in quantum statistical mechanics - an introduction. In Quantum Theory from Small to Large Scales. J. Fröhlich, M. Salmhofer, W. de Roeck, V. Mastropietro and L.F. Cugliandolo editors. Oxford University Press, Oxford, 2012.

[JOPS] Jakšić, V., Ogata, Y., Pillet, C.-A., and Seiringer, R.: Quantum hypothesis testing and non-equilibrium statistical mechanics. Rev. Math. Phys. 24, 1230002 (2012).

[JPR] Jakšić, V., Pillet C.-A, and Rey-Bellet, L.: Entropic fluctuations in statistical mechanics I. Classical dynamical systems. Nonlinearity 24, 699-763 (2011).

[JPS] Jakšić, V., Pillet, C.-A., and Shirikyan, A.: Entropic fluctuations in thermally driven harmonic networks. J. Stat. Phys. (2016) DOI 10.1007/s10955-016-1625-6

[JPW] Jakšić, V., Pillet, C.-A., and Westrich, M.: Entropic fluctuations of quantum dynamical semigroups. J. Stat. Phys. 154, 153-187 (2014).

[Jar] Jarzynski, C.: Nonequilibrium equality for free energy differences. Phys. Rev. Lett. 78, 2690 (1997).

[KM1] Kümmerer, B., and Maassen, H.: An ergodic theorem for repeated and continuous measurements. Preprint.

[KM2] Kümmerer, B., and Maassen, H.: A pathwise ergodic theorem for quantum trajectories. J. Phys. A: Math. Gen. 37, 11889-11896 (2004).

[Ku1] Kurchan, J.: Fluctuation theorem for stochastic dynamics. J. Phys. A 31, 3719-3729 (1998).

[Ku2] Kurchan, J.: A quantum fluctuation theorem. Preprint arXiv:cond-mat/0007360, (2000).

[KW] Känemäki, A., and Vilppolainen, M.: Dimensions and measures on sub-self-affine sets. Monatsh. Math. 161, 271-293 (2010). 
[LL] Landau, L.D., and Lifshitz, E.M.: Statistical Physics. Pergamon Press, Oxford, 1978.

[LN] Le Ny, A.: Introduction to (generalized) Gibbs measures. Ensaios Matematicos 15, 1-126 (2008).

[LS] Lebowitz, J.L., and Spohn, H.: A Gallavotti-Cohen-type symmetry in the large deviation functional for stochastic dynamics. J. Stat. Phys. 95, 333-365 (1999).

[Li] Lindblad, G.: Non-Markovian quantum stochastic processes and their entropy. Comm. Math. Phys. 65, 281-294 (1979).

[Ma1] Maes, C.: The fluctuation theorem as a Gibbs property. J. Stat. Phys. 95, 367-392 (1999).

[Ma2] Maes, C: On the origin and the use of fluctuation relations for the entropy. Séminaire Poincaré 2, 29-62 (2003).

[MN] Maes, C., and Netočný, K.: Time-reversal and entropy. J. Stat. Phys. 110, 269-310 (2003).

[MV] Maes, C., and Verbitskiy, E.: Large deviations and a fluctuation symmetry for chaotic homeomorphisms. Comm. Math. Phys. 233, 137-151 (2003).

[MP] Merkli, M., and Penney, M.: Quantum measurements of scattered particles. Mathematics 3, 92-118 (2015).

[MND] Mermin, N.D.: What's wrong with this pillow? Physics Today 42, 9-11 (1989).

[OP] Ohya, M., and Petz, D.: Quantum Entropy and its Use. Second edition. Springer, Berlin, 2004.

[Pe] Petz, D.: Quantum Information Theory and Quantum Statistics. Springer, Berlin, 2008.

[PS] Pólya, G., and Szegö, G.: Problems and Theorems in Analysis I. Springer, Berlin, 1978.

[RM] Rondoni, L. and Mejía-Monasterio, C.: Fluctuations in non-equilibrium statistical mechanics: models, mathematical theory, physical mechanisms. Nonlinearity 20, 1-37 (2007).

[Ro] Rockafellar, R.T.: Convex Analysis. Princeton University Press, Princeton, NJ, 1972.

[Ru1] Ruelle, D.: Thermodynamic Formalism. The Mathematical Structure of Equilibrium Statistical Mechanics. Second edition. Cambridge University Press, Cambridge, 2004.

[Ru2] Ruelle, D.: Smooth dynamics and new theoretical ideas in nonequilibrium statistical mechanics. J. Stat. Phys. 95, 393-468 (1999).

[ST] Quantum Theory: A Two Time Success Story. Yakir Aharonov Festschrift. Struppa, D.C., and Tollaksen, J.M. (eds.) Springer, Milan, 2014.

[SVW] Srivastava, Y.N., Vitiello, G., and Widom, A.: Quantum measurements, information, and entropy production. Int. J. Mod. Phys. B 13, 3369-3382 (1999).

[Ta] Tasaki, H.: Jarzynski relations for quantum systems and some applications. Preprint arXiv:cond-mat/0009244 (2000). 
[VE] van Enter, A.C.D.: On the possible failure of the Gibbs property for measures on lattice systems. Markov Proc. Rel. Fields 2, 209-224 (1996).

[VN] von Neumann, J.: Mathematical Foundations of Quantum Mechanics. Princeton University Press, Princeton, 1955.

[Wa] Walters, P.: An Introduction to Ergodic Theory. Graduate Texts in Mathematics 79. Springer, Berlin, 1982.

[Wi] Wigner, E.P.: The problem of measurement. Amer. J. Phys. 31, 6-15 (1963).

[WM] Wiseman, H. M., and Milburn, G. J.: Quantum Measurement and Control. Cambridge University Press, Cambridge, 2009.

[YK] Yi, J., and Kim, Y.W.: Nonequilibrium work by quantum projective measurments. Phys. Rev E 88, 032105 (2013).

[Ze] Zeh, H.D.: The Physical Basis of the Direction of Time. Springer, New York, 2007. 